CrossMark \& click for updates

Cite this: Polym. Chem., 2015, 6 , 2580

Received 22nd October 2014, Accepted 20th February 2015

DOI: $10.1039 / c 4 p y 01437$ e

www.rsc.org/polymers

\section{Supramolecular anticancer drug delivery systems based on linear-dendritic copolymers}

\begin{abstract}
Homa Gheybi and Mohsen Adeli*a,b
Current cancer chemotherapy often suffers severe side-effects of the administered cancer drugs on the normal tissues. In addition, poor bioavailability, due to the low water solubility of the anticancer drugs, limits their applications in chemotherapy. New delivery technologies could help overcome this challenge by improving the water solubility and achieving the targeted delivery of the anticancer drugs. Lineardendritic hybrid nanomaterials, which combine the highly branched architectures and multifunctionality of dendrimers with the processability of traditional linear-linear block copolymers, have been introduced as ideal carriers in anticancer drug delivery applications. This review presents recent advances in the investigational aspects of linear-dendritic copolymers to be applied as anticancer drug delivery vehicles. We highlight the structures, synthesis of linear-dendritic block copolymers, interaction mechanisms between linear-dendritic copolymers and anticancer drug molecules, and findings on their drug release behavior and anticancer efficacies in vitro and in vivo.
\end{abstract}

\section{Introduction}

Cancer is one of the leading causes of morbidity and accounts for approximately $20 \%$ of all deaths world-wide (World Health Organization). When a cell acquires enough mutations to become cancerous, it will be replicated at a higher rate than normal cells. After tumor mass formation, it is not possible for

${ }^{a}$ Department of Chemistry, Faculty of Science, Lorestan University, Khorramabad, Iran.E-mail:mohadeli@yahoo.comor,m.aadeli@fu-berlin.de

${ }^{b}$ Institut für Chemie und Biochemie, Freie Universität Berlin, Germany the normal cells to compete with the cancerous ones for an adequate supply of nutrients from the blood vessels. ${ }^{1-4}$ Clinical surgery to remove a cancerous tumor is considered to be the primary method for fighting cancer. However, surgery may have undesirable side effects, such as changing the growth rate of the remaining cancer cells by triggering a faster metastatic process. Following surgical resection, radiotherapy, chemotherapy and immunotherapy are the most common methods currently employed in the clinical management of cancer. ${ }^{5,6}$ One of the major problems facing cancer chemotherapy is the lack of mandatory selectivity of chemotherapeutic

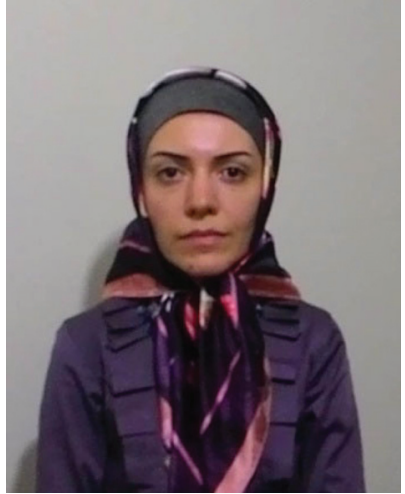

Homa Gheybi
Homa Gheybi received her BS, $M S$, and $P h D$ degrees from University of Tabriz (Iran) in 2003, 2007, and 2013, respectively. Then she continued her study in a postdoctoral research program at Lorestan University (Iran) supported by Iran Nano Council in 2014. Her research interests are nanotechnology, conducting polymers, and biodegradable drug delivery systems.

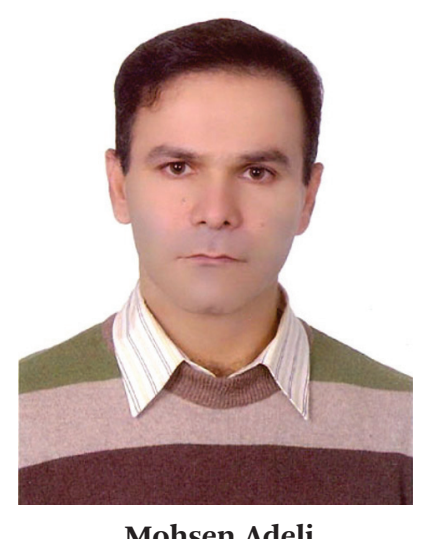

Mohsen Adeli
Mohsen Adeli received his undergraduate degree from Lorestan University (Iran) in 1996. He obtained his MS and PhD degrees from Tabriz University. After joining Lorestan University in 2005, he added a postdoctoral stage from the Institute for Nanoscience and Nanotechnology of Sharif University of Technology in 2007. He was promoted to the rank of associate and full professor in 2010 and 2013, respectively. He joined Freie Universität Berlin as a guest professor in 2014. 
drugs to direct the cytotoxicity to tumor cells, leading to undesirable side effects. ${ }^{7,8}$ A promising approach to overcome this problem is the application of nanomaterial based drug delivery systems ${ }^{9}$ including inorganic or other solid nanoparticles (gold, ${ }^{10,11}$ iron oxide, ${ }^{12,13}$ quantum dots ${ }^{14,15}$ and carbon nanotubes $\left.{ }^{16-18}\right)$, polymeric micelles, ${ }^{19-21}$ dendrimers, ${ }^{22-24}$ and liposomes. ${ }^{25,26}$ The advantages of such nanocarriers include the ability to improve drug solubility and slow down the metabolism of the drug, prolonging the circulation time, tumor specific delivery, and higher accumulation in tumors via the enhanced permeability and retention (EPR) effect, resulting in enhanced efficacy and reducing side effects. ${ }^{27-29}$ Use of nanomaterial based drug delivery systems for biomedical applications is one of the constituents of an emerging field called nanomedicine, which has shown great promise for the development of novel diagnostic, imaging, and therapeutic agents for a variety of diseases, including cancer. ${ }^{30-35}$ Among the nanomaterials, linear-dendritic hybrid materials are a growing class of nanoscopic carriers, which combine the highly branched architecture and multifunctionality of dendrimers with the processability of traditional linear-linear block copolymers. $^{36,37}$ The concept of the linear-dendritic block copolymer was announced by the Fréchet group in the early 1990s for the first time; it included a polystyrene and a dendritic poly(benzyl ether) block, ${ }^{38}$ and later on a PEG block and a hydrophobic dendritic poly(benzyl ether) block. ${ }^{39}$ Because of their unique self-assembly properties, linear-dendritic systems have received increasing attention to use as a versatile platform for drug delivery applications. ${ }^{40-42}$ Dendrimers offer plenty of advantages compared to other architectural forms of polymers that have been used in drug-delivery systems. Unique characteristics, such as highly branched globular architecture, narrow polydispersity, nanometer size range, periphery groups, physicochemical, and self-assembly properties make dendrimers promising candidates in nanomedicine. The main successes of dendrimers in nanomedicine resulted in their appropriate, reproducible and optimized design parameters, addressing the physicochemical limitations of classical drugs (e.g. solubility, specificity, stability, biodistribution and therapeutic efficiency) and their ability to overcome biological issues to reach the right target(s) (e.g. first-pass effect, immune clearance, cell penetration, off-target interactions, etc.). ${ }^{43-47}$ However, several drawbacks limit the transportation of small guest molecules by perfect dendrimers. Perfect dendrimers have a relatively rigid molecular structure with interior cavities, which are not flexible, and have certain dimensions for accepting guest molecules with a certain and defined size. For the encapsulation and release of guest molecules from perfect dendrimers, a protection and deprotection reaction series is necessary. In contrast to the drawbacks of perfect dendrimers, lineardendritic copolymers have flexible interiors and can encapsulate a variety of small guest molecules. ${ }^{48-51}$ Recently, it has been shown that the transport capacity of some of linear-dendritic copolymers is much more than that of perfect dendrimers. ${ }^{52,53}$ These advantages and interesting properties of linear-dendritic polymers have stimulated investigation in this area.
Three strategies have been reported for the synthesis of linear-dendritic copolymers ${ }^{54}$ : (a) "coupling" method: coupling of a dendron with a functionalized linear polymer; ${ }^{39}$ (b) "chain-first" method: growth of a dendritic segment from the terminal group of a linear polymer; ${ }^{55}$ (c) "dendron-first" method: polymerization of a linear segment from the dendron macroinitiator by controlled/“living" radical routes. ${ }^{56}$ These synthetic strategies lead to low polydispersity and a great deal of control over the molecular architecture of obtained lineardendritic copolymers. ${ }^{43,57-59}$

Gitsov has described detailed architectures of linear-dendritic hybrids in a valuable review. ${ }^{41}$ The building blocks of the linear-dendritic copolymers can be positioned in several distinct configurations, due to the presence of multiple anchoring points in blocks. According to Gitsov's classification, the first general group contains a single monodendron or dendrimer (D) and one (A), two (B), or multiple (C) linear segments (L), attached at the "focal" point or at the peripheral functional groups in the D fragments, Fig. 1.

The characteristic feature of the second group is the attachment of two monodendrons to the extremities of a single linear chain (E) or the incorporation of dendrimers into the main linear chain (F), Fig. 2.

The third group unifies the structures, where monodendrons are attached like "pendants" to a main linear chain through short $(\mathrm{G})$ or long spacers $(\mathrm{H})$. When the linear chain is "shrunk" $(\mathrm{H})$, it is transformed into a star-like macromolecule, with the monodendrons anchored at the extremities of the star arms (K), Fig. 3. A special case of $\mathrm{K}$ arises when the core of the star is not a small multifunctional unit, but a dendrimer.

A network constructed by dendrimers as the crosslinking moieties and linear blocks as the interjunction fragments constitutes a special case of "infinite" linear-dendritic copolymer, Fig. 4.

Finally, the dendritic-linear-dendritic super dendrimer shown in Fig. 5 represents the ultimate challenge in synthetic organic and polymer chemistry.

Besides improving the water solubility of the hydrophobic drugs, encapsulated by the hydrophobic interior of dendritic segments, ${ }^{60-62}$ the reactive surface end groups of dendritic

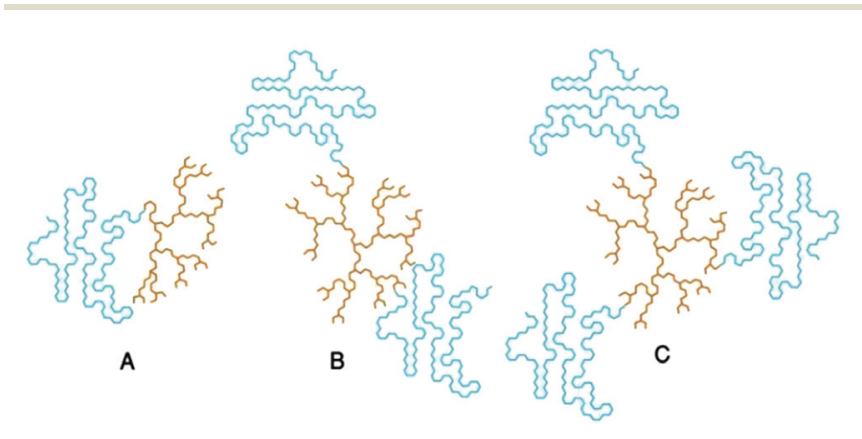

Fig. 1 Linear-dendritic architectures; first group: A, B and C. Structures were first published in ref. 41 . 


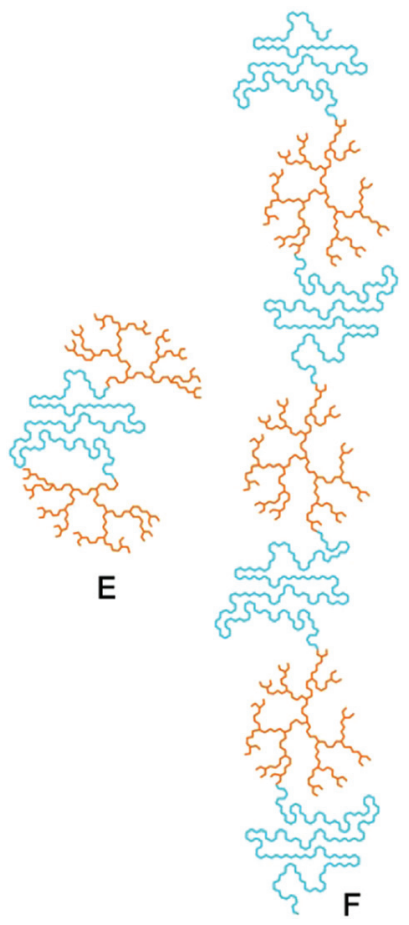

Fig. 2 Linear-dendritic architectures; second group: $E$ and F. Structures were first published in ref. 41.
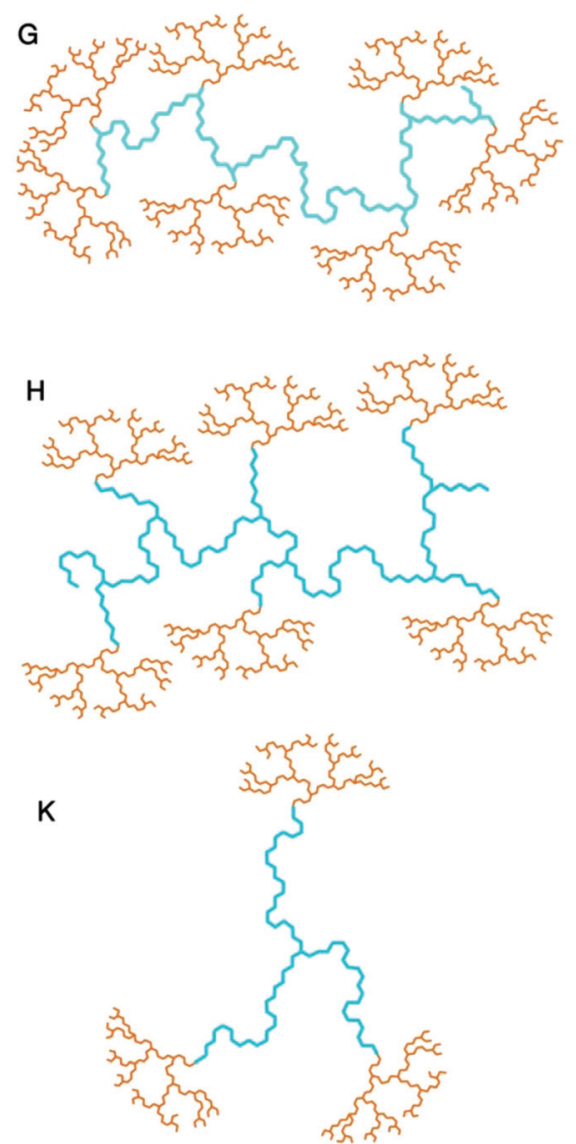

Fig. 3 Linear-dendritic architectures; third group: G, $\mathrm{H}$ and K. Structures were first published in ref. 41.

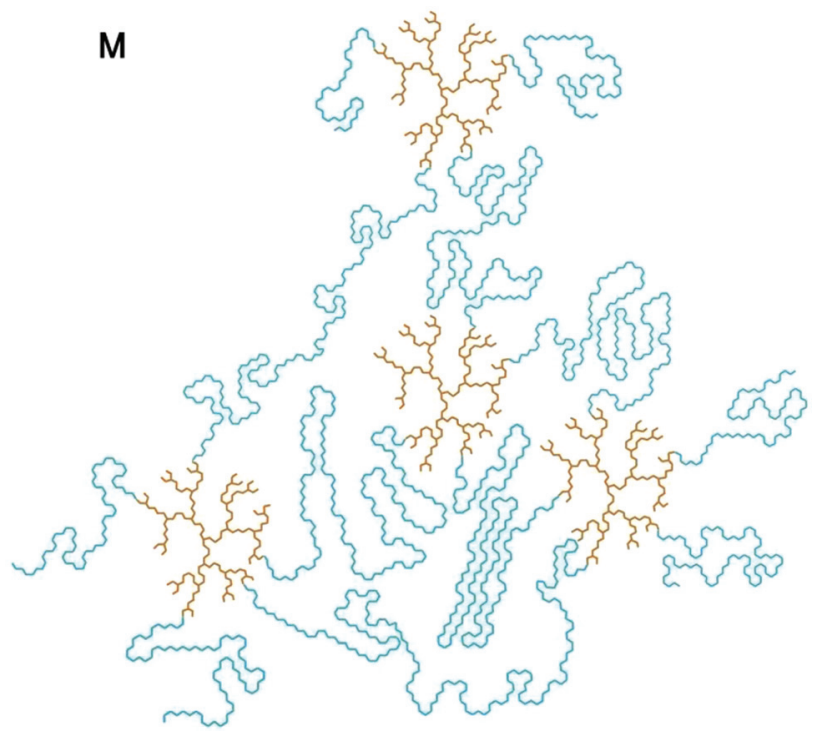

Fig. 4 Linear-dendritic architectures; fourth group: M. Structures were first published in ref. 41 .

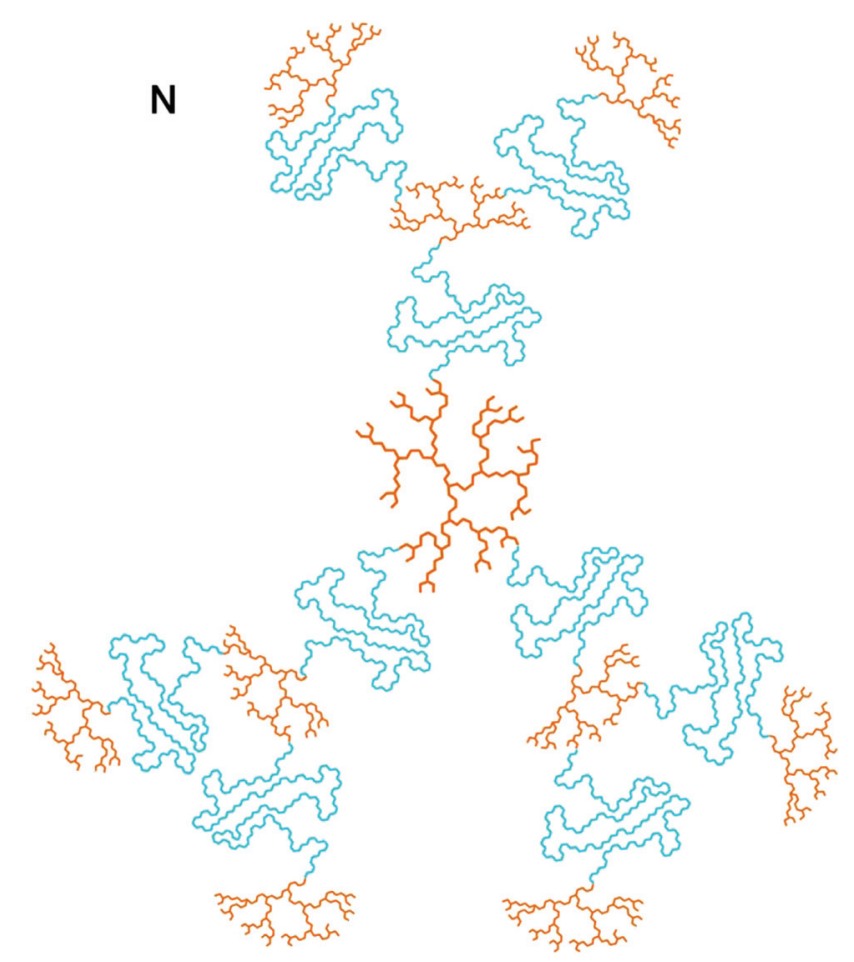

Fig. 5 Linear-dendritic architectures; fifth group: N. Structures were first published in ref. 41.

blocks can be covalently attached to a variety of drugs and therapeutic agents, ${ }^{63,64}$ targeting and imaging moieties, ${ }^{65-67}$ and bioactive molecules, ${ }^{68}$ to achieve targeting, imaging, and therapeutic treatment of cancer. Several articles reviewed the research activities generated on linear-dendritic hybrids from 
different points of view, such as discovery and synthetic strategies, PEG-dendritic block copolymer applications, and DNAprotein-dendritic biohybrids in nanobiotechnology. ${ }^{41,43,58,69,70}$ This review will focus on the potential of several (A), (E), and (K) type linear-dendritic hybrids as nanosized carriers for anticancer drug delivery systems. Specifically, we will describe the synthesis methods of related linear-dendritic hybrids, loading/ conjugating of anticancer agents onto linear-dendritic carriers, and the associated in vitro and in vivo anticancer activity.

\section{Strategies employed to load anticancer drugs onto linear-dendritic vehicles}

Currently used cancer chemotherapeutics are often inadequate to cure tumors because of the nonselectivity of these drugs, resulting in dose-limiting side effects. Strategies for reducing the toxicity and side effects without sacrificing efficacy could greatly improve treatment and quality of life for patients. Drug delivery systems that are specific to tumor cells offer both an increased therapeutic index and a reduction in harmful side effects. $^{71-73}$ Several attempts have been made to design lineardendritic copolymers as drug carriers. Drug molecules can be transferred either as conjugated to the functional groups on the dendritic structure or encapsulated by the hydrophobic interior of the dendritic blocks. These two approaches have been developed substantially, although each approach has its own advantages and drawbacks. ${ }^{74-78}$

\subsection{Linear-dendritic copolymers/drug conjugates produce vehicles}

By conjugating appropriate targeting moieties, drugs, and imaging agents to linear-dendritic polymers, 'smart' drugdelivery nanodevices can be developed that can target, deliver, and monitor the progress of therapy. ${ }^{8,79}$ However, the conjugation generally requires multi-step organic reactions and the covalent conjugation chemistry has to be optimized in order for the drug molecules to be cleaved and released under specific biological conditions. ${ }^{75}$

Drugs can be conjugated to linear-dendritic polymers, either directly or via a linker/spacer including:

- acid-labile hydrazone linkages, which are stable at physiological pH but readily cleaved under mildly acidic conditions, e.g., inside endosomes and lysosomes $;^{78}$

- ester linkages, which are hydrolyzed inside the cell by esterase enzymes; ${ }^{8}$

- disulfide bonds, which are reduced by glutathione inside the cytosol; ${ }^{80}$

- amide bond, but this bond is known to be very stable chemically in a biological environment; ${ }^{81}$

- acid sensitive acetal bonds, which are stable at $\mathrm{pH}>7$, can be an interesting option. At mildly acidic $\mathrm{pH}$, hydrolysis of the acetals is expected to occur, generating hydroxyl groups. ${ }^{82}$

\subsection{Linear-dendritic copolymers produce vehicles upon encapsulation of drugs}

In the physical encapsulated or complex form, the drugs can be loaded onto the dendritic blocks by non-covalent interactions such as van der Waals forces, hydrophobic interactions, hydrogen bonding and electrostatic interactions between oppositely charged fragments of drug molecule and dendritic blocks. ${ }^{42,74,83}$ The latter approach is relatively simple; however, in spite of significantly improving the water solubility and bioavailability of the drugs, the in vivo stability of the copolymer/drug complexes could be a challenging issue. ${ }^{75,84}$

\section{Classification based on the loaded drug}

The present review focus on the application of linear-dendritic vehicles for the delivery of four anticancer drugs: paclitaxel, doxorubicin, cisplatin, and camptothecin. The following listing of drugs associated with carriers composed of lineardendritic copolymers provides an overview of the potential of the linear-dendritic platform to serve as anticancer drug carriers (Fig. 6).

\section{Paclitaxel}

Paclitaxel (PTX), also known as Taxol (trade mark of BristolMyers-Squibb), is one of the most effective anticancer agents against a wide spectrum of cancers including ovarian, breast, and colon cancer. It exerts its antitumor effect by binding to microtubules and interfering with the normal growth of microtubules during cell division, which especially affects fast growing cancer cells. ${ }^{61,85-89}$ However, systemically administered PTX causes serious side effects, such as neutropenia and peripheral sensory neuropathy. On the other hand, to overcome its limited solubility in water, paclitaxel (Taxol) is formulated in an oily solution of Cremophor EL (polyethoxylated castor oil) and absolute ethanol $(1: 1 \mathrm{v} / \mathrm{v})$. This is known to

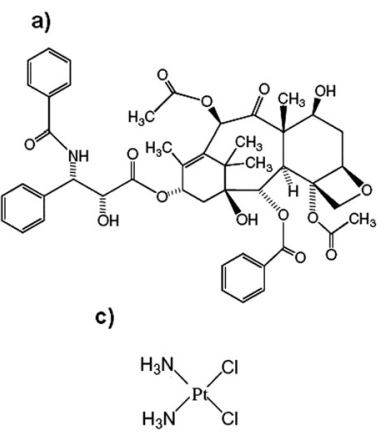

b)

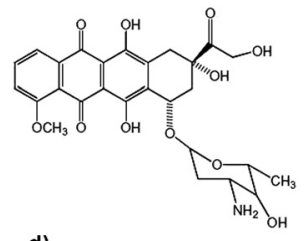

d)

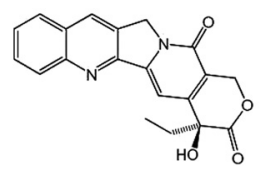

Fig. 6 Structures of (a) paclitaxel, (b) doxorubicin, (c) cis-platin, and (d) camptothecin. 
create such effects as anaphylaxis and other severe hypersensitivity reactions attributable to Cremophor EL and ethanol. ${ }^{85,86,90}$

\subsection{Paclitaxel-encapsulated linear-dendritic block copolymers}

4.1.1. Physicochemical properties. In an attempt to overcome the mentioned limitations, paclitaxel has been encapsulated into micelle-based formulations. An interesting study has reported a micellar linear-dendritic block copolymer composed of polyethylene glycol (PEG, $M_{\mathrm{w}}=5 \mathrm{KDa}$ ) and third generation polylysine terminated with 8 units of cholic acid (CA), named $\mathrm{PEG}^{5 \mathrm{~K}}-\mathrm{CA}_{8}$ telodendrimer (Fig. 7), for delivery of PTX in the treatment of nude mice bearing ovarian cancer xenografts (Compound 1). ${ }^{84}$

Physical entrapment of paclitaxel in linear-dendritic micelles has been carried out utilizing the hydrophobic interactions between PTX and the dendritic cholic acid cluster, resulting in high loading capacity $\left(7.3 \mathrm{mg}^{\mathrm{PTX}} \mathrm{mL}^{-1}\right.$ ) through the evaporation method. The particle size of $\mathrm{PEG}^{5 \mathrm{k}}-\mathrm{CA}_{8}$ nanoparticles loaded with PTX was $56 \mathrm{~nm}$, as measured by the dynamic light scattering (DLS) method. Cryo-TEM images showed that the particles were spherical in situ, and the sizes were 50-60 nm, which was consistent with the results obtained by DLS. PTX-PEG ${ }^{5 \mathrm{k}}-\mathrm{CA}_{8}$ NPs have also been found to be very stable at $4{ }^{\circ} \mathrm{C}$, showing no significant changes in average particle size over 6 months. On the other hand, Abraxane ${ }^{\circledR}$ (the FDA-approved Albumin nanoparticle-bound Paclitaxel), was unstable and started to form larger aggregates and precipitate

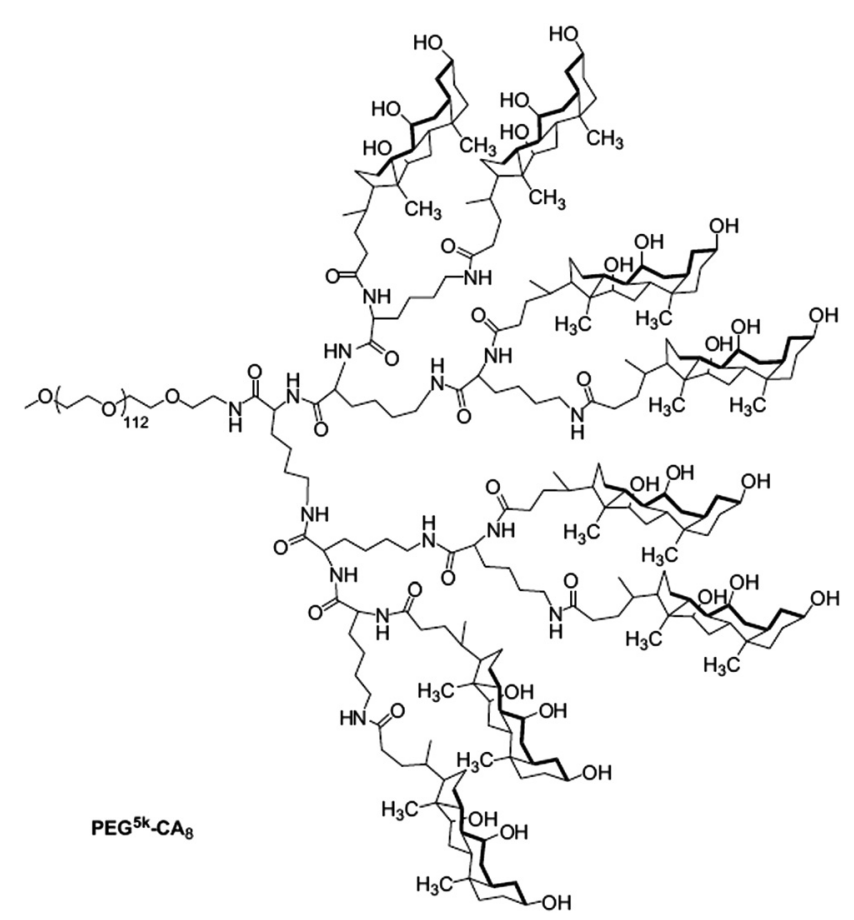

Fig. 7 The chemical structure of $\mathrm{PEG}^{5 \mathrm{~K}}-\mathrm{CA}_{8}$ (Compound 1). Reprinted with permission from ref. 84. Copyright (2009) Elsevier Ltd.
4 days after dissolving the white powder of Abraxane with saline. The obtained PTX-PEG ${ }^{5 \mathrm{k}}-\mathrm{CA}_{8}$ NPs exhibited sustained drug release into surrounding the PBS, rapid release of $20 \%$ of the drug in the first $2 \mathrm{~h}$, cumulative release of $35 \%$ of the drug by $12 \mathrm{~h}$, and a slow linear release of $75 \%$ of the drug by $156 \mathrm{~h}^{84}$ In order to better define the relationship between the structures of the $\mathrm{PEG}^{\mathrm{mk}}-\mathrm{CA}_{n}$ telodendrimers and their physicochemical properties for drug delivery, this group prepared a series of stable micelle systems with tuneable particle sizes by varying the PEG chain length and the number of cholic acids in the dendritic core. ${ }^{90}$ Other natural lipophilic molecules such as cholesterol formate (CF), lithocholic acid (LA) (both with planar steroid scaffold), and heptadecanoic acid (HA) (linear fatty acid) have also been substituted for cholic acid in the $\mathrm{PEG}^{5 \mathrm{k}}-\mathrm{CA}_{8}$ linear-dendritic structure). The resulting micelles, with low critical micelle concentrations (CMC) at approximately $1 \mu \mathrm{M}$, tended to form precipitate in aqueous solution, and their PTX loading capacities were rather low, ${ }^{90}$ indicating the essential role of facial amphiphilic cholic acid for stabilizing telodendrimer micelles. Also by varying the PEG chain length and the number of cholic acids in the dendritic core, it has been determined that larger numbers of cholic acids lead to low CMC, larger micelles with a heterogeneous size distribution, and significant precipitation after PTX loading. $\mathrm{PEG}^{5 \mathrm{k}}-\mathrm{CA}_{8}$ with a medium particle size of $61 \mathrm{~nm}$ and the highest PTX loading capacity (7.3 mg PTX loaded in $20 \mathrm{mg}$ $\mathrm{PEG}^{5 \mathrm{k}}-\mathrm{CA}_{8} \mathrm{~mL}^{-1}, 36.5 \%(\mathrm{w} / \mathrm{w})$ of drug/polymer ratio) has been found to be the optimized structure for carrying the drug. ${ }^{90}$ The stability of the PTX-loaded $\mathrm{PEG}^{5 \mathrm{k}}-\mathrm{CA}_{8}$ micelles was followed by the DLS particle sizer. The particle size of these PTXloaded micelles in aqueous solution was found to be highly stable at $4{ }^{\circ} \mathrm{C}$ for over 6 months, no further aggregations and no needle crystals of PTX were observed. As mentioned before, Abraxane tends to precipitate $24 \mathrm{~h}$ after being reconstituted with saline. Upon dilution with PBS to 125 -fold, to mimic the dilution by the blood pool through intravenous (iv) injection, needle-like crystals of PTX were observed in the diluted Taxol (Cremophor formulation of PTX) but they were not observed in PTX-loaded $\mathrm{PEG}^{5 \mathrm{k}}-\mathrm{CA}_{8}$ micelle solutions even after 12 months, indicating that the PTX complex inside these micelles is very stable.

Later Li et al. ${ }^{80}$ introduced a cross-linked micelle system for specific delivery of paclitaxel to tumor sites, composed of a dendritic oligomer of cholic acids attached to one terminus of the linear PEG through a poly(lysine-cysteine-Ebes) backbone (Compound 2). Cysteine has been inserted into the linear-dendritic structure to achieve a self assembling disulfide-crosslinking system, so that micelles can be further stabilized to avoid premature release of the loaded drugs during circulation. At the tumor sites, the intracellular reductive agents such as glutathione cleaved the intra-micellar disulfide bonds and drug release occurred. PTX loading onto the micelles was carried out by the solvent evaporation method. PTX and the polymeric structure were first dissolved in chloroform. Then, the chloroform was evaporated under vacuum to form a thin film. PBS buffer $(1 \mathrm{~mL})$ was added to re-hydrate the thin film, followed by $30 \mathrm{~min}$ sonication. The PTX-loaded micelles were 
then cross-linked via $\mathrm{O}_{2}$-mediated oxidization. The $\mathrm{CMC}$ value of $0.67 \mu \mathrm{M}$ was determined for PTX loaded cross-linked micelles. The morphology of the PTX loaded cross-linked micelles was observed to be spherical with uniform sizes under a TEM. The size of the micelles observed under TEM was consistent with those measured by DLS $(27 \mathrm{~nm}) .{ }^{80}$ The loading capacity of PTX in the cross-linked system was $7.1 \mathrm{mg}$ $\mathrm{mL}^{-1}$, which was equivalent to a drug/micelle ratio of $35.5 \%$ $(\mathrm{w} / \mathrm{w})$. PTX loaded disulfide cross-linked micelles have been found to be very stable at $4{ }^{\circ} \mathrm{C}$, showing no significant changes in average particle size and drug content over 8 months. The stability of the micelles in physiological conditions including blood was demonstrated by monitoring the particle sizes of micelles over time. The PTX loaded DCM micelles retained an average particle size around $30 \mathrm{~nm}$, with uniform and narrow size distribution, in human plasma for $24 \mathrm{~h}$. Drug release studies indicated that the PTX release was gradually facilitated as the GSH concentration increased from its extracellular level ( $2 \mathrm{mM})$ up to the intracellular level $(10 \mathrm{mM})$. This drug release strategy indicated that premature drug release can be minimized during circulation in vivo, and accelerated release occurred upon internalization of the micelles into cancer cells. $^{80}$

There is one interesting study employing biodegradable amphiphilic linear-dendritic block copolymers that present folate in clusters for cell targeting, as a means of selectively targeting drug-loaded nanocarriers for chemotherapeutics. ${ }^{91}$ PTX has been loaded into the polymeric micelles noncovalently via self-assembly of amphiphilic linear-dendritic copolymers (LDP) composed of the hydrophobic linear polypeptide block [poly $(\beta$-benzyl-L-aspartate $)]\left(M_{\mathrm{w}}=3000 \mathrm{Da}\right)$ and the hydrophilic dendritic polyester-PEG block $\left(M_{\mathrm{w}}=12000 \mathrm{Da}\right)$ (Fig. 8) (Compound 3). Folate occupied approximately $10 \%$ of the surface $(\sim 4-5$ wt $\%$ of micelle), leaving the other $90 \%$ exposed as PEG. During assembly, hydrophobic PTX is entrapped within the micelle core and the polyester-PEG dendron forms a dense antifouling shell around the micelle. The PTX-loaded micelles have a hydrodynamic diameter of $\sim 90 \mathrm{~nm}$ and a negative surface charge of $-20 \mathrm{mV}$. It was shown that PTX remained within the LDP carrier for at least 2 hours following systemic injection, which is sufficient time

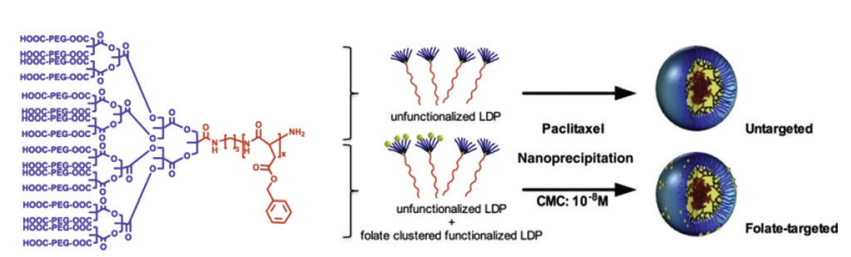

Fig. 8 Chemical structure of the linear dendritic polymer (LDP) made from biocompatible and degradable elements (linear polypeptide-[poly( $\beta$-benzyl-L-aspartate)] block and dendritic polyester-PEG block) $\left(\mathrm{X}_{n}=\right.$ 12-15). Blue, hydrophilic; red, hydrophobic. Schematic showing the preparation of paclitaxel (PTX)-encapsulated LDP micelles that do not present folate or folate clusters for enhanced cell targeting (compound 3). Reprinted with permission from ref. 91. Copyright (2011) Elsevier Inc. to allow their distribution to tumors in significant quantity via EPR. The longer stability observed can be attributed to the low micelle CMC (CMC of $\sim 10^{-8} \mathrm{M}$ ) for LDP micelles, making the system more resistant to dilution effects and destabilization by in vivo conditions. Increased effective solubility of PTX was achieved by PTX loading into the linear-dendritic micellar system with drug-loading weight efficiencies up to $40 \mathrm{wt} \%$. Drug release studies showed enhanced release at lower $\mathrm{pH}$, caused by breakdown and destabilization of the micelle structure. $^{91}$

Qiao et al. have reported a novel linear-dendritic block copolymer micelle semi-polyamidoamine- $b$-poly(D,L-lactic acid) encapsulating hydrophobic docetaxel, a semisynthetic structural analogue of paclitaxel (Compound 4). ${ }^{92}$ Hydroxyl-tailed semi-polyamidoamine dendron (sPA-OH) was synthesized by a divergent method, in which the growth of a dendron has originated from a core site. Then, ring-opening polymerization (ROP) of D,L-lactide was carried out using hydroxyl-tailed sPA-OH G4.5 (or G3.5) and a catalytic amount of Sn(Oct) 2 . DTX has been encapsulated to obtain a linear-dendritic copolymer by a co-solvent evaporation method. The hydrophobic linear PLA block formed a micelle inner core that acted as a container for insoluble drug DTX, which was subsequently stabilized by the hydrophilic capped polyamidoamine shell. The highest DTX loading efficacy (80.4\%) was achieved for the copolymer with molecular weight of $11500 \mathrm{~g} \mathrm{~mol}^{-1}$ bearing semi-PAMAM G4.5. CMC of $5.01 \mathrm{mg} \mathrm{L}^{-1}$ and a particle size of $87.4 \mathrm{~nm}$ was determined for these DTX loaded micelles. Based on drug release experiments, it was determined that approximately $100 \%$ of encapsulated DTX was released at $\mathrm{pH} 5.0$, however this value was about $75 \%$ at pHs 6.8 and 7.4 , indicating a facilitated DTX release under acidic conditions, which can be beneficial to specific drug targeting in vivo (Fig. 9). This $\mathrm{pH}$ dependence was described by the protonation of tertiary amine groups of semi-PAMAM dendritic block, which leads to conformational changes and triggers the drug release. ${ }^{92}$

4.1.2. In vitro evaluations. Investigations of in vitro anticancer activity of the PTX-PEG ${ }^{5 \mathrm{k}}-\mathrm{CA}_{8}$ NPs (Compound 1), performed on SKOV3-luc-D3 ovarian cancer cells, demonstrated similar cytotoxic activity against cancer cells as the free drug. ${ }^{84}$ In vitro cytotoxicity studies of PTX-loaded linear-dendritic micelles including $\mathrm{PEG}^{5 \mathrm{k}}-\mathrm{CA}_{8}, \mathrm{PEG}^{3 \mathrm{k}}-\mathrm{CA}_{4}$, and $\mathrm{PEG}^{3 \mathrm{k}}-\mathrm{CA}_{8}$ and the two clinical formulations of PTX (Taxol and Abraxane) on SKOV-3 cells demonstrated the $\mathrm{IC}_{50}$ in the range 4.3 to $6.2 \mathrm{ng}$ $\mathrm{mL}^{-1}$ (Fig. 10). ${ }^{90}$

Investigation of in vitro antitumor effect of PTX loaded PEG-poly(lysine-cysteine-Ebes)-cholic acid crosslinked system (Compound 2), evaluated on SKOV-3 cells, showed less cytotoxicity than Taxol. ${ }^{80}$ This was attributed to the slower release of PTX within the cell culture media. However, enhanced cytotoxicity was achieved through studies on SKOV-3 cells with an enriched GSH level, which facilitates intracellular drug release by the cleavage of intra-micellar disulfide bridges. ${ }^{80}$

Cytotoxicity studies of docetaxel encapsulated semi-polyamidoamine- $b$-poly(D,L-lactic acid) (Compound 4) on human breast cancer cell lines (MCF-7) ${ }^{92}$ showed no inhibition of 


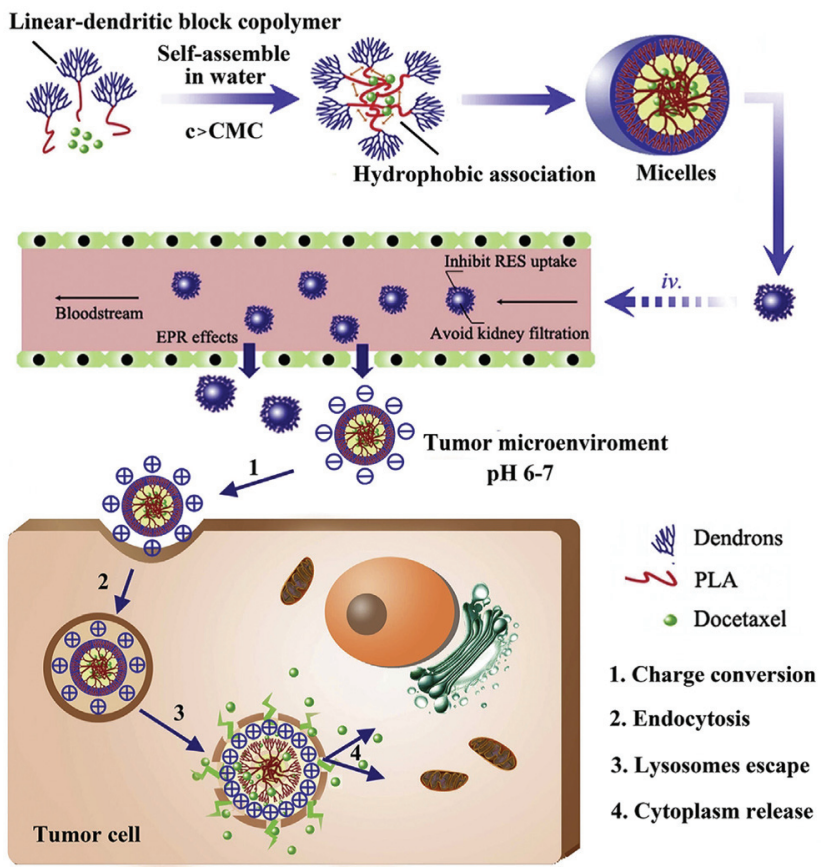

Fig. 9 Self-assembly and multifunctional target delivery of DTX-loaded polyamidoamine- $b$-poly(D,L-lactic acid) micelles (Compound 4). Reprinted with permission from ref. 92. Copyright (2013) Elsevier B.V.

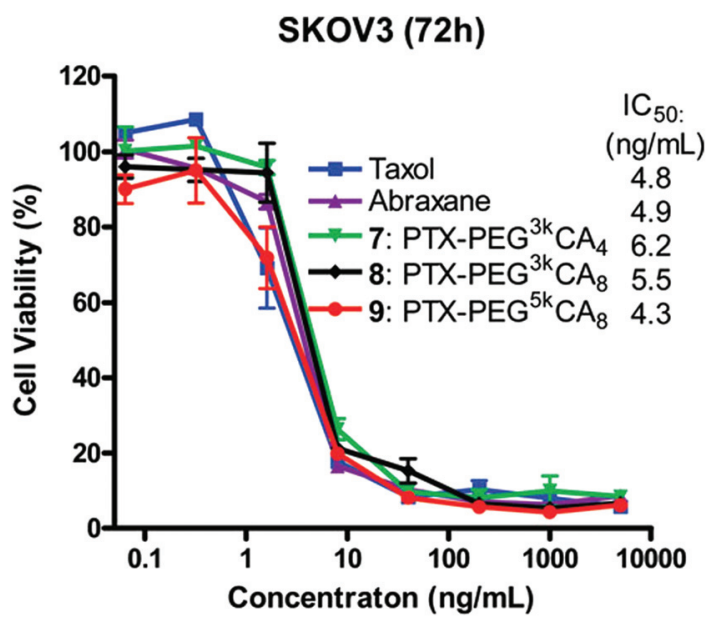

Fig. 10 Tumor cell killing of the PTX-loaded PEG ${ }^{\mathrm{mk}}-\mathrm{CA}_{n}$ micelles in the SKOV-3 ovarian cancer cells; very similar $I_{50}$ values were observed for all the formulations in in vitro tumor cell killing. Reprinted with permission from ref. 90. Copyright (2010) American Chemical Society.

cellular growth for blank micelles, attributed to reduction/ shielding of the positive charge on the dendrimer surface by the terminal ester, while DTX-loaded micelles showed equipotent anticancer efficacy as control Taxotere ${ }^{\circledR}\left(\mathrm{IC}_{50} 2.23 \pm 0.15\right.$ vs. $1.58 \pm 0.11 \mu \mathrm{g} \mathrm{mL} \mathrm{m}^{-1}$ ) (incubation time: $72 \mathrm{~h}$ ) confirming semi-PAMAM- $b$-poly(D,L-lactic acid) as a promising anticancer drug carrier. ${ }^{92}$
4.1.3. In vivo evaluations. Evaluation of the anti-tumor effects of PTX-PEG ${ }^{5 \mathrm{k}}-\mathrm{CA}_{8}$ NPs (Compound 1) after intravenous injection in subcutaneous SKOV3-luc tumor bearing mice showed inhibition of tumor growth for all PTX formulations, with inhibition being the greatest for PTX-PEG ${ }^{5 \mathrm{k}}-\mathrm{CA}_{8}$ NPs at $30 \mathrm{mg}$ PTX $\mathrm{kg}^{-1}{ }^{84}$ However, a second treatment cycle was initiated on day 38 because of noted tumor progression. A decreased tumor growth rate was demonstrated after the intravenous administration of PTX-PEG ${ }^{5 \mathrm{~K}}-\mathrm{CA}_{8}$ NPs and free drug. PTX-PEG ${ }^{5 \mathrm{k}}-\mathrm{CA}_{8}$ exhibited superior anti-tumor activity as compared with Taxol. Based on near infrared fluorescence (NIRF) imaging, it was found that PTX-PEG ${ }^{5 \mathrm{k}}-\mathrm{CA}_{8}$ nanoparticles post i.v. injected into cancer bearing mice had a prolonged blood circulation time, and preferentially accumulated in tumors, possibly as a result of EPR effects. ${ }^{84}$ In vivo biodistribution (evaluated in nude mice bearing the SKOV-3 ovarian cancer xenografts) was greatly influenced by the size of the micelles. ${ }^{90}$ Demonstrated by NIRF images, PTX-loaded PEG $^{3 \mathrm{k}}-\mathrm{CA}_{4}$ micelles with larger sizes $(154 \mathrm{~nm})$ had a very high uptake in the liver and lungs, but a low uptake in the tumor. On the other hand, $\mathrm{PEG}^{2 \mathrm{k}}-\mathrm{CA}_{4}, \mathrm{PEG}^{5 \mathrm{k}}-\mathrm{CA}_{8}$ micelles with smaller particle sizes (17 and $64 \mathrm{~nm}$ ) showed a better ability to carry the loaded drug to the tumor sites, which was attributed to EPR effects. In vivo antitumor effects for PTX-loaded $\mathrm{PEG}^{5 \mathrm{k}}-\mathrm{CA}_{8}$ was found to be superior to those of Abraxane and Taxol, and cure of the disease was achieved in the group treated with PTXloaded $\mathrm{PEG}^{5 \mathrm{k}}-\mathrm{CA}_{8}$ at its MTD dosage $\left(45 \mathrm{mg} \mathrm{kg}^{-1}\right)$. No weight loss was observed in mice treated with this nanoformulation, while consistent weight loss was observed in those treated with Taxol. ${ }^{90}$ This system has some significant advantages, such as nontoxicity of carrier in contrast to the commercial vehicle of PTX, high drug loading capacity, relatively small sustained drug release profile, superior stability, preferential accumulation in tumors, similar in vitro cytotoxic activity with Taxol, and superior in vivo anti-tumor activity as compared with Taxol. ${ }^{84,90}$ Enhanced efficacy of PTX encapsulated PEG ${ }^{5 \mathrm{k}}$ $\mathrm{CA}_{8}$ NPs after intravenous injection can be explained as follows: First, the $\mathrm{PEG}^{5 \mathrm{k}}-\mathrm{CA}_{8}$ nanocarrier may improve the pharmacokinetic profile of PTX, prolonging its circulation time, thus resulting in a higher accumulation in tumors due to EPR effects. Secondly, since PTX encapsulated $\mathrm{PEG}^{5 \mathrm{k}}-\mathrm{CA}_{8}$ NPs accumulate in the tumor, PTX is released in a sustained manner so that tumor cells can be exposed to PTX for a longer time period. Thirdly, PTX encapsulated $\mathrm{PEG}^{5 \mathrm{k}}-\mathrm{CA}_{8}$ NPs have a relatively small size $(50 \mathrm{~nm})$ compared to Abraxane $(130 \mathrm{~nm})$, which may result in deeper penetration into tumor nodules. This is important as although some larger nanoparticles, such as liposomes, can be delivered effectively to a solid tumor via the EPR effect, they would not be distributed sufficiently to cancer cells distant from tumor vessels. ${ }^{93}$ These promising features make PTX encapsulated $\mathrm{PEG}^{5 \mathrm{k}}-\mathrm{CA}_{8}$ NPs suitable for more investigations. ${ }^{84,90}$

For the PTX encapsulated PEG-poly(lysine-cysteine-Ebes)cholic acid crosslinked system (Compound 2), ${ }^{80}$ according to noninvasive NIRF optical images, a significant contrast of the fluorescence signal has been observed between the tumor and 
the background at $4 \mathrm{~h}$ after administration and was sustained up to $72 \mathrm{~h}$ (Fig. 11, top). Ex vivo imaging at $72 \mathrm{~h}$ post injection further confirmed the preferential uptake of crosslinked

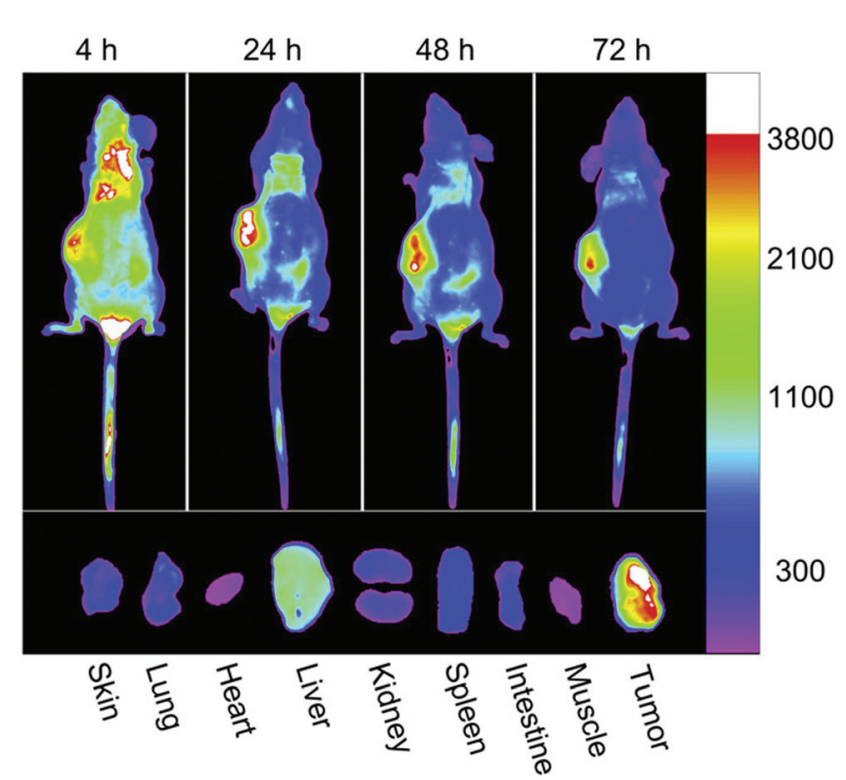

Fig. 11 In vivo and ex vivo near infra-red fluorescence (NIRF) optical imaging. Top: In vivo NIRF optical images of a SKOV-3 xenograft bearing mouse were obtained with a Kodak imaging system at different time points after i.v. injection of PEG-poly(lysine-cysteine-Ebes)-Cholic acid co-loaded with PTX and DiD; bottom: ex vivo NIR image of dissected organs and tumor was obtained at $72 \mathrm{~h}$ after injection. Reprinted with permission from ref. 80. Copyright (2011) Elsevier Ltd. micelles in the tumor compared to normal organs (Fig. 11, bottom). This is due to the prolonged in vivo circulation time of the micelles and the size mediated EPR effect. A relatively high uptake in the liver was observed compared to other organs, which is likely attributed to the nonspecific clearance of nanoparticles by the reticuloendothelial system (RES). Similar biodistribution and tumor uptake of PTX loaded crosslinked micelles were observed via EPR effects (Table 1). ${ }^{80}$

Based on in vivo studies evaluated in the subcutaneous SKOV-3 tumor bearing mice, increased in vivo therapeutic efficacy has been shown for PTX-loaded crosslinked micelles compared to the equivalent dose of free drug. This has been corresponded to the higher amount of PTX that reached the tumor site via their prolonged circulation time. In addition, the high glutathione level of the tumor site and particularly inside the tumor cells facilitated drug release and increased cytotoxicity. ${ }^{80}$ Uniform size around of $27 \mathrm{~nm}$ for PTX encapsulated the PEG-poly(lysine-cysteine-Ebes)-cholic acid crosslinked system enables these PTX loaded micelles to take full advantage of the EPR effect and accumulate at tumor sites. In addition, the system has the characteristics of superior drug loading capacity, enhanced micellar stability, prolonged in vivo circulation time, preferential tumor targeting, and superior in vivo anti-tumor activity as compared with Taxol. However, a relatively high uptake in the liver was observed compared to other organs, which is likely attributed to the nonspecific clearance of nanoparticles by the reticuloendothelial system (RES). ${ }^{80}$

In the case of polymeric vehicle composed of hydrophobic linear polypeptide block [poly( $\beta$-benzyl-L-aspartate $)]$ and hydro-

Table 1 Paclitaxel-encapsulated linear-dendritic block copolymers

\begin{tabular}{|c|c|c|c|}
\hline Carrier & $\begin{array}{l}\text { Size } \\
(\mathrm{nm})\end{array}$ & $\mathrm{CMC}$ & In vitro activity \\
\hline $\mathrm{PEG}^{5 \mathrm{~K}}-\mathrm{CA}_{8}$ & 56 & $1 \mu \mathrm{M}$ & $\begin{array}{l}\mathrm{IC}_{50}=4.3 \mathrm{ng} \mathrm{mL} \mathrm{m}^{-1} \text { on SKOV3- } \\
\text { luc-D3 ovarian cancer cells }\end{array}$ \\
\hline $\begin{array}{l}\text { PEG-poly (lysine- } \\
\text { cysteine-Ebes)-CA }\end{array}$ & 27 & $0.67 \mu \mathrm{M}$ & $\begin{array}{l}\text { Less cytotoxicity compared to } \\
\text { Taxol on SKOV-3 cells, but } \\
\text { higher cytotoxicity compared } \\
\text { to Taxol with an enriched } \\
\text { GSH level }\end{array}$ \\
\hline
\end{tabular}

$[\operatorname{poly}(\beta$-benzylL-aspartate)]polyester-PEG

$90 \quad 10^{-8} \mathrm{M}$

Semi-PAMAM$b$-poly(D,L-lactic acid)
$87.4 \quad 5.01 \mathrm{mg} \quad \mathrm{IC}_{50}=2.23 \pm 0.15 \mu \mathrm{g} \mathrm{mL} \mathrm{mL}^{-1}$ on $\mathrm{L}^{-1} \quad$ human breast cancer cell lines (MCF-7), equipotent anticancer efficacy as control Taxotere

In vivo activity
Superior anti-tumor activity
for PTX-PEG ${ }^{5 \mathrm{k}}-\mathrm{CA}_{8}$ as
compared with Taxol after
intravenous injection in
subcutaneous SKOV3-luc
tumor bearing mice

Increased in vivo therapeutic efficacy compared to the equivalent dose of free drug in the subcutaneous SKOV-3 tumor bearing mice

Higher in vivo antitumor efficacy for the folate-bearing linear-dendritic micelles compared to free PTX in FR-expressing KB xenograft model in nude mice

1.53 fold higher half-life of

DTX in micelles compared to Taxotere ${ }^{\circledR}$
Advantages

Ref.

High drug loading level, nontoxic carrier, high drug loading capacity, relatively small sustained drug release profile, superior stability, preferential accumulation in tumors, superior anti-tumor activity as compared with Taxol

Superior drug loading capacity, enhanced micellar stability, prolonged in vivo circulation time, preferential tumor targeting, and superior in vivo anti-tumor activity as compared with Taxol

Suitable circulation half-life, resistance to dilution effects and destabilization by in vivo conditions, increased targeting of the micelles to FR-expressing cells, increased accumulation Biocompatibility, improved cellular uptake, facilitated anticancer drug release under acidic conditions

\section{4 ,} 90

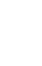


philic dendritic polyester-PEG block (Compound 3), ${ }^{91}$ after intravenous injection in BALB/c mice without tumors, distribution half-lives $\left(t_{1 / 2}\right.$, distribution $)$ of the encapsulated PTX (2.5 wt $\%)$ and free PTX (2.5 wt\% equivalent) were $1.72 \pm$ 0.2 hour, and $0.61 \pm 0.4$ hour, and their elimination half-lives $\left(t_{1 / 2}\right.$, elimination $)$ were $9.06 \pm 2$ hours, and $4.32 \pm 3$ hours, respectively, indicating a much higher bioavailability of the PTXloaded LDP micelles compared to the free drug. This can be attributed to the low CMC of micelles, making the system more resistant to dilution effects and destabilization by in vivo conditions. Also $10-12 \%$ weight loss was revealed in mice receiving free-PTX and signs of hair loss were observed after six injections. No signs of toxicity were shown in mice receiving PTX-encapsulated injections. In vivo antitumor efficacy investigations, evaluated in the FR-expressing KB xenograft model in nude mice, showed that the folate-bearing lineardendritic micelles (PTX dosage $=2.5 \mathrm{mg} \mathrm{kg}^{-1}$ ) are as effective as a higher dose of free PTX $\left(10 \mathrm{mg} \mathrm{kg}^{-1}\right)$. Comparison of the tumor killing effects between folate-targeted and untargeted micelles (PTX dosage $=2.5 \mathrm{mg} \mathrm{kg}{ }^{-1}$ ) determined the higher antitumor efficacy for the folate-targeted LDP system. This was attributed to more efficient entering of the folate targeted micelles into tumor cells from the extracellular space, through FR-mediated uptake, and subsequent intracellular release of the drug. ${ }^{91}$ The measured circulation half-life of PTX encapsulated $[\operatorname{poly}(\beta$-benzyl-L-aspartate $)]$-block-dendritic polyester-PEG was significantly higher than the values reported in preclinical trials for PTX loaded PEG-block-poly(D,L-lactide) linear block copolymers in the literature. ${ }^{94}$ The longer stability, due to low micelle CMC, more resistance to dilution effects and destabilization by in vivo conditions, increased targeting of the micelles to FR-expressing cells, increased accumulation over a 5-day period, and the potency of the therapy with a low PTX dose of $2.5 \mathrm{mg} \mathrm{kg}^{-1}$ make this system promising for antitumor treatment. For achieving a relatively similar effect, this dosage regimen is much lighter in comparison to other studies involving folate-mediated therapy. ${ }^{95-97}$ However, the data also suggest a gradual loss of PTX from the micelles at longer time points via slow leakage of the drug from the interior of the micelle, and future design of the system may be applied to further address this issue. ${ }^{91}$

Pharmacokinetic study in Sprague-Dawley (SD) rats illustrated that semi-polyamidoamine- $b$-poly(D,L-lactic acid) micelles (Compound 4) prolonged DTX retention in blood circulation $(1.737 \mathrm{~h})$ in comparison to Taxotere ${ }^{\circledR}$ (commercial vehicle of DTX) (1.231 h), and the half-life of DTX in micelles was 1.53 fold higher than that of the Taxotere ${ }^{8}$ control. ${ }^{92} \mathrm{~A}$ plausible explanation was that ester-terminated hydrophilic dendrons and a dense micelle structure were capable of preventing the drug molecules from being easily eliminated from the physiological environment. Investigation of intracellular uptake capacity evaluated in MCF-7 cells demonstrated that fluorescent dye C6 labeled semi-polyamidoamine- $b$-poly(D,Llactic acid) micelles had been internalized into the cytoplasm. Previous studies indicated that multivalent dendrimers enhanced membrane adhesion and disruption. ${ }^{98}$ The multi- valent dendritic surface of linear-dendritic semi-polyamidoamine- $b$-poly(D,L-lactic acid) might also increase binding sites with the membrane and facilitate micelle uptake. Further studies are needed to elucidate the uptake mechanisms of semi-polyamidoamine- $b$-poly(D,L-lactic acid) micelles. Generally, low CMC would be beneficial to semi-polyamidoamine- $b$ poly(D,L-lactic acid) micelles in order to avoid dilution in the blood circulation. Some other advantages such as biocompatibility, improved cellular uptake, and facilitated anticancer drug release under acidic conditions make linear-dendritic semi-polyamidoamine- $b$-poly(D,L-lactic acid) useful for anticancer drug delivery. However, its safety and efficacy in chemotherapy should be further studied. ${ }^{92}$

\subsection{Paclitaxel-conjugated linear-dendritic block copolymers}

4.2.1. Physicochemical properties. A linear-dendritic targeting system for PTX delivery has been reported by Clementi et al., ${ }^{8}$ employing alendronate (ALN), a bone-targeting agent used for the treatment of osteoporosis and bone metastases. A PEG-dendrimer-ALN structure has been designed using $\beta$-glutamic acid as a symmetric bicarboxylic branching unit and linking of ALN to carboxylic PEG-dendrimer groups. Coupling of PTX to PEG-dendrimer-ALN through ester linkage yielded the linear-dendritic ALN-mediated bone targeting prodrug (Compound 5) (Fig. 12).

This design leads to an amphiphilic conjugate, with PTX being highly hydrophobic and ALN being hydrophilic. The spatial separation of these drugs, besides offering the possibility to form self-assembled micelles, will maintain all ALN molecules exposed to the water, promptly available for binding to the bone mineral HA. ${ }^{8}$ The content of ALN and PTX in the PEG-( $\beta$-Glu $)$ dendrimer was determined as $11 \% \mathrm{w} / \mathrm{w}$ and $4.7 \%$ $\mathrm{w} / \mathrm{w}$, respectively. The mean hydrodynamic diameter of PTXPEG-( $\beta$-Glu) dendrimer-ALN conjugates was $200 \mathrm{~nm}$. Investigation of drug release showed that the hydrolysis rate of the ester bond between PTX and the polymer was higher in both

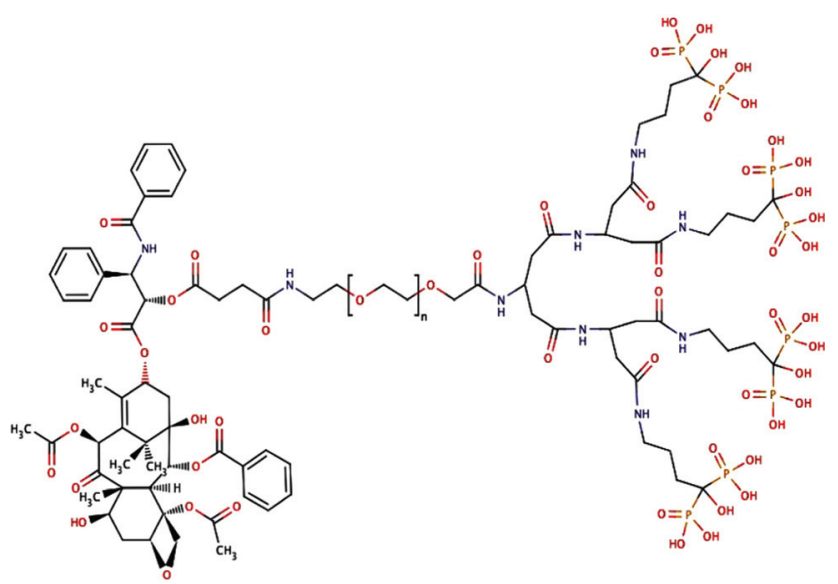

Fig. 12 Chemical structure of PTX conjugated PEG-( $\beta$-Glu) dendrimer-ALN (Compound 5). Reprinted with permission from ref. 8. Copyright (2011) American Chemical Society. 
Table 2 Paclitaxel-conjugated linear-dendritic block copolymers

\begin{tabular}{|c|c|c|c|c|c|c|}
\hline Carrier name & Size & CMC & In vitro activity & $\begin{array}{l}\text { In vivo } \\
\text { activity }\end{array}$ & Advantages & Ref. \\
\hline $\begin{array}{l}\text { PEG-poly } \\
\text { ( } \beta \text {-glutamic } \\
\text { acid)-ALN }\end{array}$ & 200 & $\mathrm{n}^{a}$ & 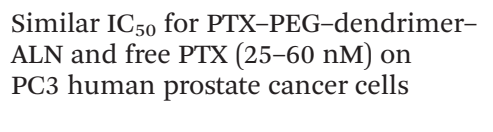 & $\mathrm{n}^{a}$ & $\begin{array}{l}\text { Nontoxic carrier building blocks, half-life } \\
\text { prolongation of conjugates, targeting bone } \\
\text { neoplasms, fast drug release in the surroundings of } \\
\text { bone metastasis }\end{array}$ & 8 \\
\hline
\end{tabular}

${ }^{a} \mathrm{n}$ : not reported.

plasma and buffer solution at physiological pH (7.4), compared to that in buffer solution at lysosomal $\mathrm{pH}(\mathrm{pH}$ ). This phenomenon indicated that PTX is released by a hydrolytically based mechanism without a significant contribution of esterases. The stability of PTX-PEG-( $\beta$-Glu) dendrimer-ALN was evaluated in buffer solutions at physiological $\mathrm{pH}$ (7.4), at lysosomal pH (5), and in mice plasma. At pH 7.4 and in plasma, about $50 \%$ of the PTX-PEG-ALN conjugate was degraded within the first $1 \mathrm{~h}$; the remaining conjugate was degraded within $24 \mathrm{~h}$. The stability of the conjugate micelles, monitored at $37^{\circ} \mathrm{C}$ for $24 \mathrm{~h}$ by DLS, was in line with the kinetics of PTX release. The micelles of PTX-PEG-ALN conjugates preserved the same size for $24 \mathrm{~h}$ when incubated in buffer at $\mathrm{pH} 5$, whereas at $\mathrm{pH} 7.4$ the same micelles were stable for $3 \mathrm{~h}$, then the PTX release from the conjugates destabilized the system. ${ }^{8}$

4.2.2. In vitro evaluations. ALN-mediated binding capacity of PEG-( $\beta$-Glu) dendrimer-ALN and PTX conjugated PEG( $\beta$-Glu) dendrimer-ALN (Compound 5) to bone mineral was evaluated in vitro, employing HA mineral mimicking bone tissue. ${ }^{8}$ By fast protein liquid chromatography (FPLC) analysis, it was revealed that, following $5 \mathrm{~min}$ of incubation, $80 \%$ or 90\% of PTX-PEG-dendrimer-ALN or PEG-ALN conjugates, respectively, were bound to $\mathrm{HA}$ and reached a plateau. On the other hand, non-targeting PEG could not bind to HA after 60 min of incubation, confirming the role of ALN in the bone targeting process.

Illustrated by in vitro rat red blood cell (RBC) hemolysis assay, PTX-PEG-( $\beta$-Glu) dendrimer-ALN showed no hemolytic activity at up to $5 \mathrm{mg} \mathrm{mL} \mathrm{m}^{-1}$. Regarding significant hemolytic activity of the commercial solubilizing vehicle of PTX, PEG( $\beta$-Glu) dendrimer-ALN can be suggested as a promising carrier for PTX. In vitro cytotoxicity assay on PC3 human prostate cancer cells exhibited similar IC $_{50}$ for PTX-PEG-dendrimer-ALN and free PTX (25-60 nM). ${ }^{8}$

4.2.3. In vivo evaluations. The pharmacokinetic studies of PTX dissolved in 1:1:8 ethanol-Cremophor EL-saline and PTX-PEG-( $\beta$-Glu) dendrimer-ALN (Compound 5) showed an improved Pharmacokinetic Profile in Mice. ${ }^{8}$ After administration of free PTX, high levels of the drug were recorded, however at $5 \mathrm{~min}$ post-injection the PTX concentration decreased dramatically, and it was not detectable at $60 \mathrm{~min}$. On the contrary, the conjugates showed a marked half-life prolongation, with detectable levels of PTX after $24 \mathrm{~h}$ for
PTX-PEG-( $\beta$-Glu) dendrimer-ALN. In particular, elimination half-lives $\left(T_{1 / 2 \beta}\right)$ were 15.1 and 85.5 min for PTX and PTX-PEG( $\beta$-Glu) dendrimer-ALN, respectively. ${ }^{8}$

PTX-PEG-( $\beta$-Glu) dendrimer-ALN conjugate was designed for strong bone tropism and fast drug release. Therefore, with PTX-PEG-( $\beta$-Glu) dendrimer-ALN conjugate, a cathepsin B-cleavable linker might not be suitable because the derivative in vivo will bind to the bone HA matrix. The high affinity to the bone originating from the presence of a bisphosphonate in the conjugate can affect the conjugate internalization into cancer cells and consequently slow the rate of PTX release, if a cathepsin B-cleavable linker is used. Cathepsin B is over-expressed in lysosomes of many types of tumor cells, but is also secreted to the extracellular matrix. In general, enzymatic cleavage is efficient when slow and controlled drug release is required. When a fast release is desired, a different mechanism, such as hydrolysis, is necessary. Therefore, a PTX-polymer hydrolysis at physiological conditions is preferred because it allows drug release in the surroundings of bone metastasis, where the conjugate will fast accumulate.

PTX was linked to PEG through an ester linkage exploiting a succinimidyl spacer, which releases the drug at physiological $\mathrm{pH}$. The hydrolysis rate of the ester bond between the drug and the polymer was higher in buffer at $\mathrm{pH} 7.4$ than in $\mathrm{pH}$ 5. Interestingly, the incubation in plasma showed a drug release comparable to that in buffer at $\mathrm{pH}$ 7.4, suggesting that PTX is released by a hydrolytically based mechanism without a significant contribution of esterases. Besides nontoxic building blocks, the derivative can target bone neoplasms by dualtargeting as follows: (1) through ALN (active mechanism), and (2) by exploiting the EPR effect (passive mechanism), which is due to the atypically leaky tumor blood vasculature, which enhances tumor accumulation of the conjugate thanks to its increased size with respect to the free drug (Table 2). ${ }^{8}$

\section{Doxorubicin}

Doxorubicin (DOX) is a widely used anticancer drug in the treatment of many types of cancer, including hematological malignancies, many types of carcinoma, and soft tissue sarcomas. DOX is known to interact with DNA by intercalation and to inhibit the biosynthesis of macromolecules..$^{67,99-101}$ 
However, drawbacks such as poor water solubility, poor penetration in vitro and in vivo, and dose dependent sideeffects such as cardiotoxicity, caused by lack of tumor selectivity, limit its application in chemotherapy. ${ }^{101-103}$ Several techniques have been used to enhance tumor targeting and reduce the toxicity without sacrificing efficacy. The use of macromolecular drug carriers such as liposomes, ${ }^{104}$ polymeric micelles, ${ }^{105}$ dendrimers ${ }^{106}$ and linear-dendritic copolymers ${ }^{107}$ is the focus of research. One clinical example is DoxilvR, a polyethylene glycol (PEG) containing liposomal formulation of DOX that limits the cardiotoxicity while maintaining the same survivability as the free drug. ${ }^{102}$

\subsection{Doxorubicin-encapsulated linear-dendritic block copolymers}

5.1.1. Physicochemical properties. Gillies and Fréchet have reported DOX encapsulation in acid-sensitive linear-dendritic micelles. The linear-dendritic structure was composed of a PEO block and a G3 polyester dendrimer of 2,2-bis(hydroxymethyl) propanoic acid units bearing cyclic acetals of 2,4,6trimethoxybenzaldehyde (Compound 6 in Fig. 13). DOX was loaded into micelles by an oil/water emulsion method in which chloroform was used as the organic phase, and 3 equiv $\mathrm{NEt}_{3}$ was used relative to DOX, as the drug is known to partition most effectively into the chloroform phase and into the micelle upon deprotonation of the glycosidic amine. Release of the drug occurred through disruption of the micelle caused by hydrolysis of the cyclic acetals at acidic $\mathrm{pH}$ and change in micelle solubility. ${ }^{107}$

A drug loading of approximately $12 \mathrm{wt} \%$ was determined according to absorbance using UV-visible spectroscopy. The DOX-loaded micelles demonstrated the particle size of $35 \mathrm{~nm}$ and a small fraction of aggregates in the size range 200-400 nm was observed. Additionally, a CMC value of $40 \mathrm{mg}$ $\mathrm{L}^{-1}$ was determined for this system. At $\mathrm{pH} 7.4$, the micelles were stable over several days, with no significant changes in the size distribution over this time period. In contrast, over several hours at $\mathrm{pH} 5.0$, the size of the DOX-loaded micelles

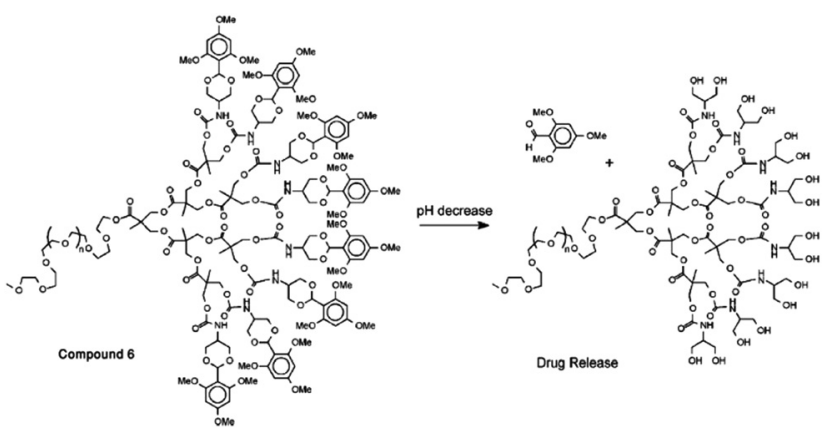

Fig. 13 Hydrolysis of acetals on the dendrimer periphery of the micelle-forming copolymer 6 leads to a solubility change designed to disrupt micelle formation and trigger the release of the drug. Reprinted with permission from ref. 107. Copyright (2005) American Chemical Society. increased and the fraction of aggregates in the population became greater. This aggregation probably occurred upon disruption of the micelles due to acetal hydrolysis and was facilitated by the tendency of DOX to form aggregates by $\pi$-stacking. Investigation of drug release at different $\mathrm{pH}$ values demonstrated a pH-dependent mode of release of DOX from copolymer 1 . After $24 \mathrm{~h}$, drug release of more than $80 \%$, about $40 \%$ and about $30 \%$ was observed at $\mathrm{pH}$ values of 4.0, 5.0 and 6.0, respectively, while at $\mathrm{pH} 7.4$ the system was very stable with less than $10 \%$ of the DOX being released over $72 \mathrm{~h}$. These results indicated that the hydrolysis of the $\mathrm{pH}$-sensitive acetals likely plays a role in the mode of drug release, leading to the selective release of DOX in mildly acidic physiological environments (such as tumor tissues). In addition, the increased stability of the $\mathrm{pH}$-sensitive system at $\mathrm{pH} 7.4$ is advantageous so that DOX will not be released during blood circulation, thus avoiding the undesirable organ accumulation and toxicity associated with the free drug. ${ }^{107}$

A newly developed telodendrimer platform, consisting of PEG as the linear block and cholic acid attached to the amine terminus of lysine as the dendritic block, has been employed to prepare DOX micellar formulations for the targeted delivery of DOX to lymphoma. ${ }^{108}$ As mentioned before, ${ }^{84} \mathrm{PEG}^{5 \mathrm{k}}-\mathrm{CA}_{8}$ micellar NPs provided a suitable drug delivery system for PTX (a hydrophobic drug) in the treatment of cancer. In their study, Xiao et al. showed that the delivery of DOX using this nanocarrier is limited by the relatively low drug loading capacity and poor stability. ${ }^{108}$ Using the dry-down method, $\mathrm{PEG}^{5 \mathrm{~K}}-\mathrm{CA}_{8}$ telodendrimers can efficiently encapsulate hydrophobic drug DOX into the core of the micelles (Compound 7). $\mathrm{PEG}^{5 \mathrm{k}}-\mathrm{CA}_{8}$ telodendrimer, along with different amounts of neutralized DOX, were first dissolved in $\mathrm{CHCl}_{3}-\mathrm{MeOH}$, mixed, and evaporated on a rotavapor to obtain a homogeneous dry polymer film. The film was reconstituted in $1 \mathrm{~mL}$ phosphate buffered solution (PBS), followed by sonication for $30 \mathrm{~min}$, allowing the sample film to disperse into the micelle solution. $^{108}$

Based on their report, $\mathrm{PEG}^{5 \mathrm{k}}-\mathrm{CA}_{8}$ micelle was found to have a DOX loading capacity of $8.2 \% \mathrm{w} / \mathrm{w}$. Another telodendrimer with a similar structure, $\mathrm{PEG}^{2 \mathrm{k}}-\mathrm{CA}_{4}$, was suggested to encapsulate DOX, which resulted in a higher drug loading capacity of $14.8 \% \mathrm{w} / \mathrm{w}$. The particle sizes of DOX-loaded $\mathrm{PEG}^{5 \mathrm{k}}-\mathrm{CA}_{8}$ and $\mathrm{PEG}^{2 \mathrm{k}}-\mathrm{CA}_{4}$ micelles were in the range $12-17 \mathrm{~nm}$ in diameter. Drug release profiles illustrated biphasic patterns for both DOX-loaded $\mathrm{PEG}^{2 \mathrm{k}}-\mathrm{CA}_{4}$ and $\mathrm{PEG}^{5 \mathrm{k}}-\mathrm{CA}_{8}$ micellar formulations. It was indicated that $50 \%$ DOX cumulative release occurred from DOX-PEG ${ }^{5 \mathrm{k}}-\mathrm{CA}_{8}$ during the first $6 \mathrm{~h}$. This value was $35 \%$ for $\mathrm{DOX}-\mathrm{PEG}^{2 \mathrm{k}}-\mathrm{CA}_{4}$ over the same time. After the initial fast release, the slow linear release was observed for both systems over the next 7 days. However, the DOX release rate from DOX$\mathrm{PEG}^{2 \mathrm{k}}-\mathrm{CA}_{4}$ micelles was significantly slower than that from DOX-PEG ${ }^{5 \mathrm{k}}-\mathrm{CA}_{8}$ micelles, reflecting the better stability and stronger interaction between the nanocarrier and the drug in DOX-PEG ${ }^{2 \mathrm{k}}-\mathrm{CA}_{4}$. Particle size monitoring confirmed the stability of DOX-PEG ${ }^{2 \mathrm{k}}-\mathrm{CA}_{4}$ in physiological conditions. As shown by DLS measurements (Fig. 14), DOX-PEG ${ }^{2 \mathrm{k}}-\mathrm{CA}_{4}$ micelles as 


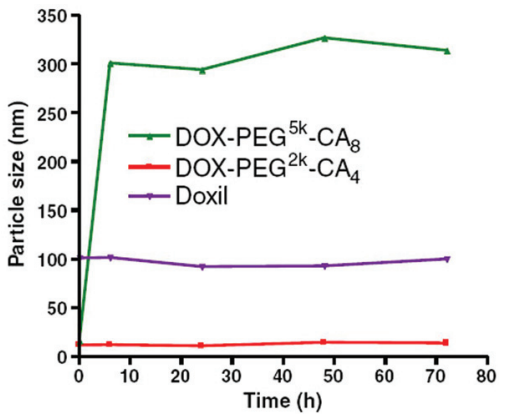

Fig. 14 DLS measurement of particle size change of DOX-loaded $\mathrm{PEG}^{5 \mathrm{k}}-\mathrm{CA}_{8}$ micelles, and Doxil@ in $50 \%$ FBS over time at $37{ }^{\circ} \mathrm{C}$. DOX loading level was $2 \mathrm{mg} \mathrm{ml}^{-1} \mathrm{DOX}$ in $20 \mathrm{mg} \mathrm{ml}^{-1}$ telodendrimer, respectively. Reprinted with permission from ref. 108. Copyright (2011) Elsevier B.V.

well as Doxil@ were able to maintain their initial particle sizes over $72 \mathrm{~h}$ incubation in the presence of $50 \%$ FBS. In contrast, DOX-PEG ${ }^{5 \mathrm{k}}-\mathrm{CA}_{8}$ micelles started to form bigger aggregates (around $300 \mathrm{~nm}$ ) after a 6-hour incubation with 50\% FBS. ${ }^{108}$

Wu et al. developed dendritic-linear block copolymer-modified magnetic iron oxide nanoparticles as a carrier for DOX, which displays thermosensitive drug release behaviors. ${ }^{109}$ In their study, magnetic iron oxide $\left(\mathrm{Fe}_{3} \mathrm{O}_{4}\right)$ nanoparticles were first prepared by organic solution-phase decomposition of the iron precursor at high temperature. The prepared magnetic iron oxide nanoparticle surfaces were capped by the propargyl focal point polyamidoamine (PAMAM)-type dendron, having four carboxyl acid end groups. Then, by a click reaction, the surface initiator was introduced onto the propargyl group, and using two-step surface-initiated ATRP, poly(2-dimethylaminoethyl methacrylate) (PDMAEMA) chains and poly( $\mathrm{N}$-isopropylacrylamide) (PNIPAM) chains were sequentially introduced onto the magnetic nanoparticle surfaces resulting in PAMAM- $b$-PDMAEMA- $b$-PNIPAM block copolymer-modified magnetic iron oxide nanoparticles. At the final step, to increase stability of the nanoparticles and reverse aggregation, a cross-linking reaction between PDMAEMA block and 1,2-bis(2-iodoethoxy)ethane (BIEE) was carried out (Fig. 15). After the crosslinking reaction, the magnetic nanoparticles were stabilized in water, forming a stable brown solution and no precipitation occurred for 4 months. However, non-crosslinked $\mathrm{Fe}_{3} \mathrm{O}_{4}$ PAMAM- $b$-PDMAEMA- $b$-PNIPAM nanoparticles could be only stabilized in water for 2 months. It was also verified that the cross-linking reaction could be helpful to stabilize magnetic iron oxide nanoparticles. DLS measurements of freshly crosslinked block copolymer-modified nanoparticles diluted in water provided an average hydrodynamic diameter of particles in solution equal to $\sim 32 \mathrm{~nm}$. DOX has been loaded into the PAMAM- $b$-PDMAEMA- $b$-PNIPAM shell of the modified nanoparticles with a loading efficiency of $22.7 \%$ (Compound 8). ${ }^{109}$

Investigation of drug release behaviour of DOX-loaded nanoparticles demonstrated the cumulative release amounts of $26.8 \%$ and $13.7 \%$ at $25^{\circ} \mathrm{C}$ and $37^{\circ} \mathrm{C}$, respectively $(\mathrm{pH}=7.4$,
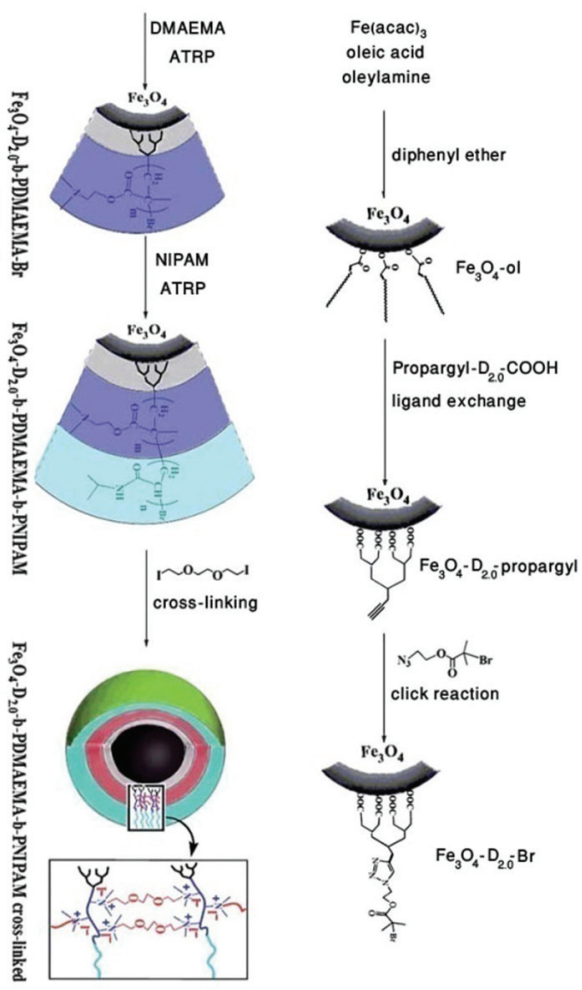

Fig. 15 Synthesis and surface modification of superparamagnetic $\mathrm{Fe}_{3} \mathrm{O}_{4}$ nanoparticles (Compound 8). Reprinted with permission from ref. 109. Copyright (2011) The Royal Society of Chemistry.

$5 \mathrm{~h}$ ). This phenomenon was also observed after $24 \mathrm{~h}$ with the cumulative release amounts of $41 \%$ and $26 \%$ at $25{ }^{\circ} \mathrm{C}$ and $37{ }^{\circ} \mathrm{C}$, respectively. It was noted that the cumulative release amount was higher at $25^{\circ} \mathrm{C}$ than at $37^{\circ} \mathrm{C}$, confirming thermosensitive release caused by PNIPAM block chains in the collapsed and hydrophobic conformation at $37^{\circ} \mathrm{C}$ above the LCST, which can retard drug release. ${ }^{109}$

Also, superparamagnetic iron oxide nanoparticles (SPIONs), grafted with a DOX-loaded water-soluble dendritic-linearbrush-like triblock copolymer, polyamidoamine- $b$-poly(2-(dimethylamino)ethyl methacrylate)- $b$-poly(poly(ethylene glycol) methyl ether methacrylate) (PAMAM- $b$-PDMAEMA- $b$-PPEGMA), have been reported as a pH-sensitive drug delivery system (Compound 9). ${ }^{110}$ Immobilization of an ATRP macroinitiator, containing PAMAM G2-typed dendron, on the surface of $\mathrm{Fe}_{3} \mathrm{O}_{4}$ nanoparticles has been carried out according to the reported procedure in the literature. ${ }^{109}$ Then, water soluble dendriticlinear-brush-like triblock copolymer (PAMAM- $b$-PDMAEMA- $b$ PPEGMA)-grafted SPIONs have been prepared by gradual growing of PDMAEMA and PPEGMA from nanoparticle surfaces using the ATRP "grafting from" approach. After removal of the $\mathrm{Fe}_{3} \mathrm{O}_{4}$ cores with hydrochloric acid, $M_{\mathrm{n}}$ and PDI of the grafted copolymers PAMAM- $b$-PDMAEMA-Br were $3900 \mathrm{~g} \mathrm{~mol}^{-1}$ and 1.08 , respectively. For the final grafted copolymers PAMAM- $b$-PDMAEMA- $b$-PPEGMA, $M_{\mathrm{n}}$ and PDI were $26300 \mathrm{~g}$ $\mathrm{mol}^{-1}$ and 1.25 , respectively. DLS measurement confirmed 
that the obtained dendritic-linear-brush-like triblock copolymer-grafted SPIONs had a uniform hydrodynamic particle size of average diameter less than $30 \mathrm{~nm}$. For drug loading, DOX$\mathrm{HCl}(2.0 \mathrm{mg})$ was dissolved in methanol $(4.0 \mathrm{~mL})$, and triethylamine $(25 \mu \mathrm{L})$ was then added into the solution to remove hydrochloride. Drug loading was done by drop-wise addition of the DOX solution with stirring to $3 \mathrm{~mL} \mathrm{Fe}_{3} \mathrm{O}_{4}$-PAMAM- $b$ PDMAEMA- $b$-PPEGMA nanoparticles in methanol (concentration of $2.5 \mathrm{mg} \mathrm{mL}^{-1}$ ). The mixture was shaken for $24 \mathrm{~h}$ in the dark at room temperature to allow the drug partition into the polymer shell. The modified $\mathrm{Fe}_{3} \mathrm{O}_{4}$-PAMAM- $b$-PDMAEMA$b$-PPEGMA nanoparticles possessed the thicker shell of polymers, which is beneficial to enhance hydrophobic interactions and hydrogen binding with DOX and improve the loading capacity. ${ }^{110}$ In vitro drug release experiments showed a $\mathrm{pH}$ responsive drug release behavior for DOX-loaded nanoparticles. The cumulative release amounts of DOX within $48 \mathrm{~h}$ at pH 4.7, 7.4 and 11.0 are $83.1 \%, 64.7 \%$ and $8.3 \%$, respectively. The higher release at $\mathrm{pH} 4.7$ has been attributed to decreased hydrogen bond interaction between PDMAEMA and DOX due to the protonation of PDMAEMA chains and DOX at $\mathrm{pH}$ 4.7. On the other hand, PDMAEMA chains tend to swell due to the protonated tertiary amino groups at $\mathrm{pH} 4.7$, which is beneficial to accelerate DOX release. ${ }^{110}$

The antitumor effect of doxorubicin encapsulated into amphiphilic linear-dendritic hybrids of PEG-Gn-PCL $(n=0,1$, 2) (Compound 10) has been evaluated in MDA-MB-231 and MDA-MB-468 breast adenocarcinomas. ${ }^{102}$ The linear component of PEG has been employed as the hydrophilic block with a dendron branched poly( $\varepsilon$-caprolactone) (PCL) as the hydrophobic one. The dendrons have been prepared from the 2,2-bis(methylol)propionic acid (bisMPA) building block, bearing click chemistry moieties in the focal point to attach PEG (Fig. 16).

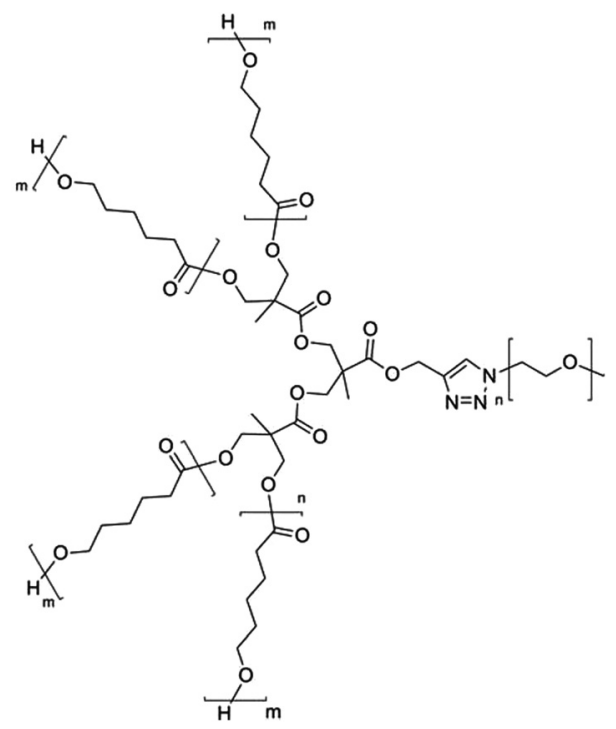

Fig. 16 Chemical structure of PEG-G2-PCL (Compound 10). Reprinted with permission from ref. 102. Copyright (2011) Wiley Periodicals, Inc.
The amphiphilic PEG-Gn-PCL structures were capable of forming self-assembled micelles with diameters of about $100 \mathrm{~nm}$. Sequestering doxorubicin achieved a loading efficiency up to $22 \%$ for PEG2k-G1-PCL30. It has been demonstrated that the loading of DOX resulted in aggregation of the smaller particles into larger ones, suggesting that some PEG components used in these linear-dendritic hybrids are not sufficiently large to effectively shield the particle from particleparticle interactions. Also increasing the generation of the dendron to two resulted in a substantial loss of loading efficiency down to $8 \%$ (PEG5k-G2-PCL30). ${ }^{102}$ This has been attributed to the effect of lower crystallinity, due to the effect of the dendritic branching, resulting in a less densely packed hydrophobic core. Also, the incorporation of DOX in a nanoscale confined crystalline core will reduce the crystallinity further. Drug release studies revealed that the mode of release was independent of block portion and dendrimer generation employed in the micelle construction, suggesting that the core material did not influence the diffusion path of the drug from the core. The release of about $60 \%$ at $6 \mathrm{~h}$ and more than $80 \%$ at $24 \mathrm{~h}$ has been shown for all evaluated systems. ${ }^{102}$

Recently carbon nanotubes have been introduced as promising materials for the delivery of drugs, RNA, DNA, peptides and other biologically active molecules into cells, because of the ability to cross cell membranes. ${ }^{111-114}$ Despite the great potential of carbon nanotubes (CNTs) in anticancer drug delivery, concerns regarding their carcinogenicity, inefficient dispersion in aqueous solutions and biological activity in vivo still remain. One important and feasible route to overcome these problems is modification of CNTs with polymers, which are widely studied and play a vital role in biological and biomedical fields, especially in drug delivery. ${ }^{115}$ Two methods are used to modify the CNTs by polymers based on either physical interactions or chemical bonding and are so called "noncovalent" or "covalent" approaches, respectively. The noncovalent approach is based on poor van der Waals interactions between CNTs and polymers and includes dispersion with the low molar mass polymers, polymer wrapping and polymer adsorption. ${ }^{116-118}$ In the covalent approach, molecules or macromolecules are grafted onto the surface of CNTs through chemical linkages, raising the solubility of CNTs even with a low degree of functionalization. Covalent attachment of polymer chains to the surface of CNTs can be accomplished by either "grafting to" or "grafting from" methods. ${ }^{114,115,119-124}$ We proved that polymers not only raise the functionality, biocompatibility and water solubility of CNT but also are able to change the CNT conformations dramatically. A drug carrier composed of polyglycerol-poly(ethylene glycol)-polyglycerol (PG-PEG-PG) ABA linear-dendritic copolymer and CNTs was designed and potential application of the obtained structure to load and transport the anticancer drug DOX was investigated (Compound 11). ${ }^{125}$ Fig. 17 illustrates the schematic structure of the used linear-dendritic copolymer. It has been observed that noncovalent interactions between (PG-PEG-PG) linear-dendritic copolymer and CNTs lead to the conformation alteration of CNTs from an extended toward a closed state due 


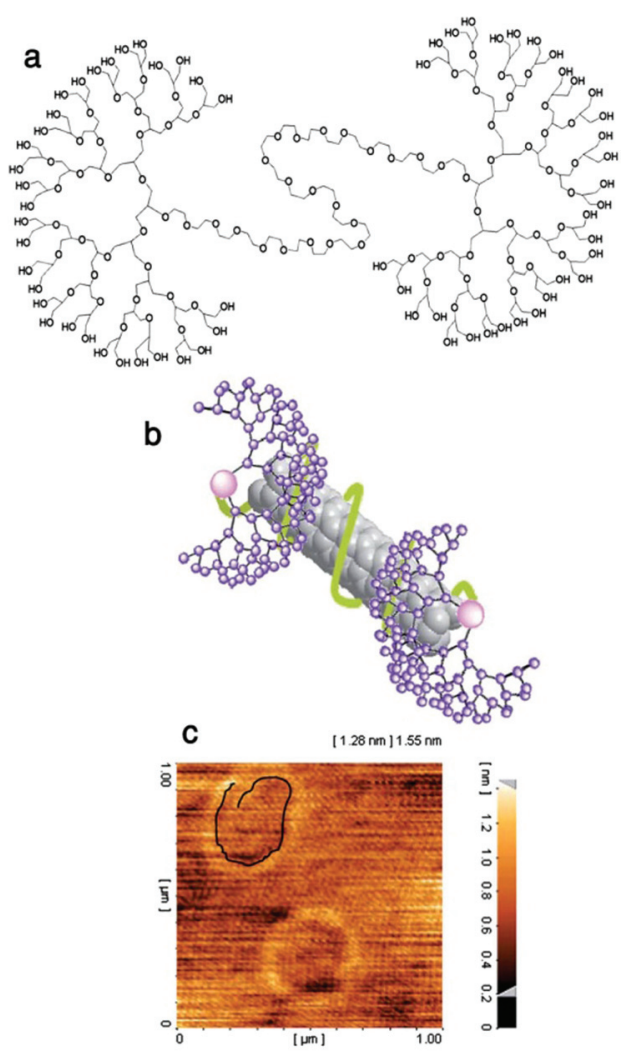

Fig. 17 (a) Schematic representation of PG-PEG-PG ABA type linear dendritic copolymer, (b) schematic representation of noncovalent interactions between linear-dendritic copolymers and the surface of CNTs that lead to new hybrid nanomaterials with improved properties, (c) AFM image of PG-PEG-PG/MWCNT liposome-like nanocapsules containing encapsulated DOX molecules. The highlighted object in the top-left part of image, by a black line, shows the MWCNTs (Compound 11). Reprinted with permission from ref. 125. Copyright (2012) The Royal Society of Chemistry.

to liposome-like nanocapsule (LLNs) formation. The size of the PG-PEG-PG/MWCNT LLNs filled with DOX molecules was estimated to be $350 \mathrm{~nm}$.

Loading capacities for the PG-PEG-PG/MWCNT LLNs were determined by HPLC as 2.2 grams to one gram of LLNs. ${ }^{125}$

Poor water solubility and low functionality are two critical factors that limit the biomedical applications of $\mathrm{CNT} /$ $\gamma-\mathrm{Fe}_{2} \mathrm{O}_{3} \mathrm{NP}$ hybrid nanomaterials. Non-covalent method for improving solubility is based on supramolecular interactions between CNTs and polymers and includes polymer wrapping or adsorption. In this method, the structure of CNTs is not damaged as much as in the covalent method, but its disadvantage is the low functionality of the final product. A new method to improve the functionality and water solubility of $\mathrm{CNT} / \gamma-\mathrm{Fe}_{2} \mathrm{O}_{3} \mathrm{NP}$ hybrid nanomaterials without damaging their structure has been reported by using linear-dendritic copolymers. $^{126-128}$

An example of non-covalent interactions between CNTs and linear-dendritic copolymers is hybrid nanostructure-based magnetic drug delivery systems (HNMDDSs), including carbon nanotubes, magnetic iron oxide nanoparticles, and linear-dendritic PAMAM-PEG-PAMAM copolymer. $^{32}$ PAMAM-PEGPAMAM was employed to solubilize and functionalize carbon nanotubes through supramolecular interactions. The resulting $\mathrm{Fe}_{3} \mathrm{O}_{4}$-MWCNTs/PAMAM-PEG-PAMAM hybrid nanomaterials were utilized to encapsulate DOX (through $\pi-\pi$ stacking) with a loading capacity of about 3.3\% (Compound 12). According to dynamic light scattering, the average diameter of DOX loaded $\mathrm{Fe}_{3} \mathrm{O}_{4}$-MWCNTs/PAMAM-PEG-PAMAM nanomaterials in water was $207 \mathrm{~nm}$ and aqueous solutions of hybrid nanomaterials were stable over several weeks at room temperature. ${ }^{32}$

Based on VSM curves, the saturation of magnetization of $\mathrm{Fe}_{3} \mathrm{O}_{4}$-MWCNTS/PAMAM-PEG-PAMAM and DOX/Fe ${ }_{3} \mathrm{O}_{4^{-}}$ MWCNTS/PAMAM-PEG-PAMAM were slightly smaller than that of $\mathrm{Fe}_{3} \mathrm{O}_{4}$-MWCNTs, but both had similar properties that were close to the superparamagnetic behavior, indicating that the magnetic properties of $\mathrm{Fe}_{3} \mathrm{O}_{4}$-MWCNTs did not lose by the non-covalent interaction of DOX and PAMAM-PEG-PAMAM on their surfaces. With this unique property, $\mathrm{DOX} / \gamma-\mathrm{Fe}_{3} \mathrm{O}_{4}$ MWCNTS/PAMAM-PEG-PAMAM can be used as a promising material in many fields such as cancer diagnosis and therapy (Fig. 18). ${ }^{32}$

Using copper(I)-catalyzed azide-alkyne cycloaddition (CuAAC)-based click chemistry and "dendrone-first" method, Hed et al. synthesized a set of dendritic linear hybrid materials composed of linear PEG and dendritic aliphatic bis-MPA polyesters (G4). ${ }^{129}$ To achieve amphiphilic structures, the bis-MPA layer also introduced hydrophobicity, using benzylidene-protected bis-MPA anhydride. Finally, the convergent coupling was carried out between monofunctional PEG5k-acetylene and azide functional dendrons azide-[G4]-(Bz) 8 by CuAAC click chemistry (Fig. 19).

Increased sizes of the micelles has been demonstrated after encapsulation of DOX into the amphiphilic PEG-[G4]-(Bz) 8 (diameters reported for the intensity average DLS data: $88 \mathrm{~nm}$ for PEG-[G4]-(Bz) 8 , and $300 \mathrm{~nm}$ for DOX loaded PEG-[G4]-(Bz)

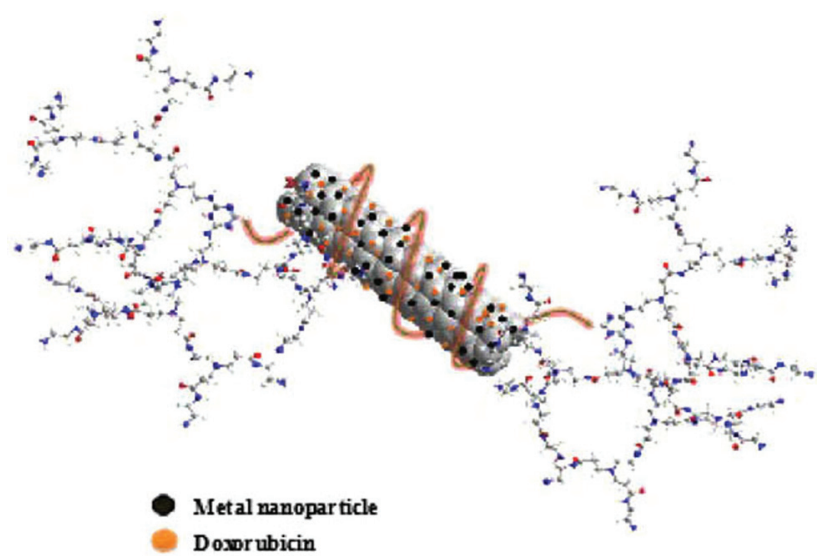

Fig. 18 The schematic representation of HNMDDSs including carbon nanotubes, magnetic iron oxide nanoparticles, and linear-dendritic PAMAM-PEG-PAMAM copolymer (Compound 12). Reprinted with permission from ref. 32. Copyright (2013) Iranian Chemical society. 


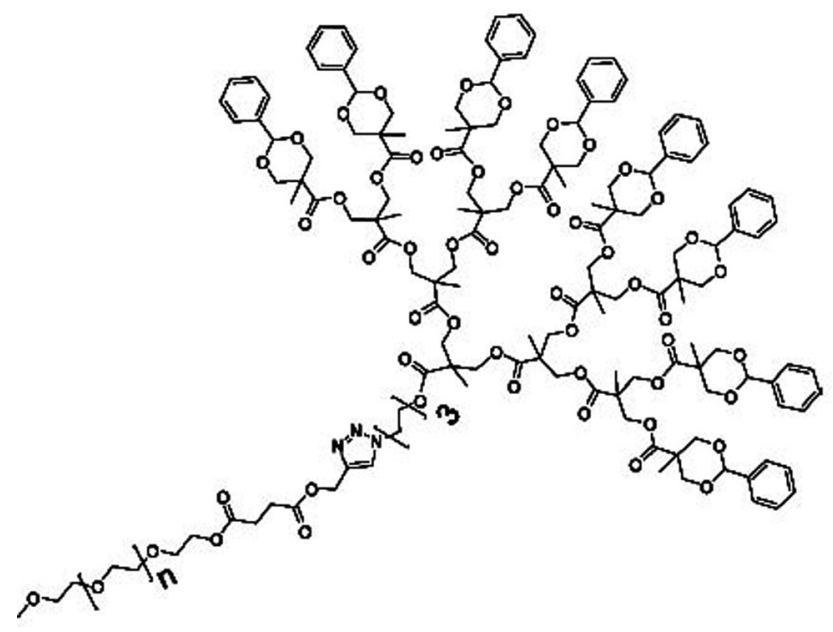

Fig. 19 Schematic structure of DL PEG-[G4]-(Bz) 8 (Compound 13). Reprinted with permission from ref. 129. Copyright (2013) Wiley Periodicals, Inc.

(Compound 13)) indicating that the PEG length used for these materials is not sufficient to suppress aggregation. A loading efficiency of $51 \%$ has been found for DOX-loaded PEG-[G4]$(\mathrm{Bz})_{8}$. Drug release studies exhibited a burst release during the first $12 \mathrm{~h}$, under which at least $80 \%$ of the DOX was released, and around $90 \%$ of the content was released within $72 \mathrm{~h}$. This phenomenon has been attributed to the low capacity of the hydrophobic domain to act as a diffusion barrier. It was suggested that increasing the PEG molecular weight should be noted to restrict aggregation and achieve better controlled release kinetics. ${ }^{129}$

Recently, the stimuli-responsive polymeric micelles and assemblies that are triggered by the light as external stimuli have been widely investigated for "on-off" drug delivery systems and "on-demand" nanomedicines, because of their assembly/disassembly switch. Compared with UV light, the near infrared (NIR) light between 750 and $1000 \mathrm{~nm}$ can penetrate up to a centimetre in depth of the tissues with less damage and scattering, which makes the NIR-sensitive nanomedicines promising as noninvasive and on-demand therapeutic candidates. ${ }^{130,131}$

Sun et al. have reported near-infrared (NIR) light-responsive linear-dendritic amphiphiles consisting of linear PEO and dendritic PAMAM (third-generation, D3) decorated with diazonaphthoquinone (DNQ) employed for NIR-triggered release of the anticancer drug doxorubicin (Compound 14). ${ }^{132}$ In their synthetic route, the reaction between the alkyne focal point PAMAM Dendron (D3: bearing eight primary amine groups) and DNQ sulfonyl chloride has been utilized to yield the clickable dendron D3DNQ (with eight DNQ groups), which has then been click conjugated with azide-terminated PEO (5 K) to produce the final linear-dendritic amphiphiles (Fig. 20). It has been demonstrated that under NIR (e.g. $808 \mathrm{~nm})$ irradiation, the hydrophobic diazonaphthoquinone (DNQ) molecule transforms into the hydrophilic photoproduct 3-indenecarboxylic

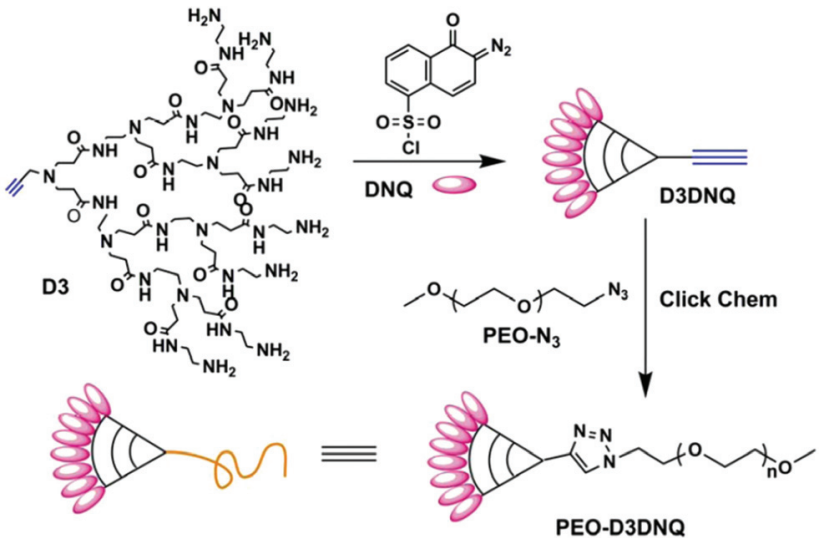

Fig. 20 Synthesis of linear-dendritic amphiphiles PEO-PAMAM (D3) DNQ by click chemistry (Compound 14). Reprinted with permission from ref. 132. Copyright (2013) The Royal Society of Chemistry.

acid $\left(\mathrm{p} K_{\mathrm{a}}=4.5\right)$ via the Wolff rearrangement, ${ }^{132}$ which would result in the disassembly and/or disruption of DNQ-containing micelles in PBS (e.g. $\mathrm{pH} 7.4) .{ }^{133-135}$

Besides having a common spherical morphology, PEO5KD3DNQ micelles had a DLS-determined diameter of $\sim 90 \mathrm{~nm}$. In addition, the average size of the micelles slightly changed over 25 days in PBS $(10 \mathrm{mM}, \mathrm{pH} 7.4)$ at $37^{\circ} \mathrm{C}$, suggesting that they were dynamically stable in vitro. A DOX-loading capacity of about $20 \mathrm{wt} \%$ has been determined for PEO5K-D3DNQ micelles. Determined by DLS, the DOX-loaded PEO5K-D3DNQ micelles increased from $90 \mathrm{~nm}$ (blank micelles) to $160 \mathrm{~nm}$. These results also suggested that the hydrophobic DOX drug was indeed encapsulated into the hydrophobic core of the micelles. Similar to their blank counterparts, the DOX-loaded micelles presented a nearly spherical morphology. In vitro drug release studies demonstrated a NIR-triggered drug release profile for DOX-loaded PEO5K-D3DNQ micelles. The accelerated drug-release was exhibited after $10 \mathrm{~min}$ of $808 \mathrm{~nm}$ irradiation. It was found that $90 \%$ of DOX was released within about $210 \mathrm{~h}$, compared with that without irradiation (about $420 \mathrm{~h}$ ). Moreover, the apparent drug-release rate of DOXloaded PEO5K-D3DNQ micelles accelerated nearly 8 times after $30 \mathrm{~min}$ of $808 \mathrm{~nm}$ irradiation compared to 10 min radiation, indicating NIR-responsive DOX release from the nanomedicines. ${ }^{132}$

5.1.2. In vitro evaluations. Based on in vitro cytotoxicity investigations evaluated on MDA-MB-231 breast cancer cells, $\mathrm{IC}_{50}$ of approximately $3 \mu \mathrm{g} \mathrm{mL}^{-1}$ and $0.8 \mu \mathrm{g} \mathrm{mL}^{-1}$ has been determined for DOX-loaded PEO-block-G3 polyester dendrimer of 2,2-bis(hydroxymethyl)propanoic acid units bearing cyclic acetals of 2,4,6-trimethoxybenzaldehyde micelles (Compound 6) and free drug, respectively. ${ }^{107}$ The somewhat lower toxicity of the micelle system may result from the gradual release of DOX within the cell and from differences in the released drug's cellular localization relative to the free drug. However, $\mathrm{IC}_{50}$ of $3 \mu \mathrm{g} \mathrm{mL}{ }^{-1}$ for DOX-loaded micelles indicated the release of free and active DOX in the cells and indicated 
encouraging therapeutic potential of the system. Drug localization in intracellular organelles has been proved for DOXloaded micelles by Laser scanning confocal microscopy images, while free DOX was localized in the cell nucleus after $24 \mathrm{~h}$ (Fig. 21). ${ }^{107}$ Drug localization in intracellular organelles has been proved for DOX loaded micelles by Laser scanning confocal microscopy images. As shown in Fig. 21a, MDAMB-231 cells exposed to free DOX show significant accumulation of DOX in the nucleus after $24 \mathrm{~h}$. In contrast, cells exposed to DOX-loaded $\mathrm{pH}$-sensitive micelles have a punctate fluorescence, which is concentrated in the cytoplasm after 24 h, as shown in Fig. 21b. These observations are important for several reasons. First, the absence of DOX fluorescence in the nucleus suggests that the micelles are stable in the presence of cells and serum-containing cell medium, as the rapid destabilization of the micelles in the extracellular environment and subsequent release of DOX outside the cell would be expected to result in an image similar to that observed for free DOX. In addition, the fluorescence in the cytoplasm suggests that the DOX-loaded micelles are indeed taken up by cells, and its punctate nature is consistent with the localization of the drug in subcellular organelles. ${ }^{107}$ The increased stability of the $\mathrm{pH}$-sensitive system at $\mathrm{pH} 7.4$, and controlled release of thera-
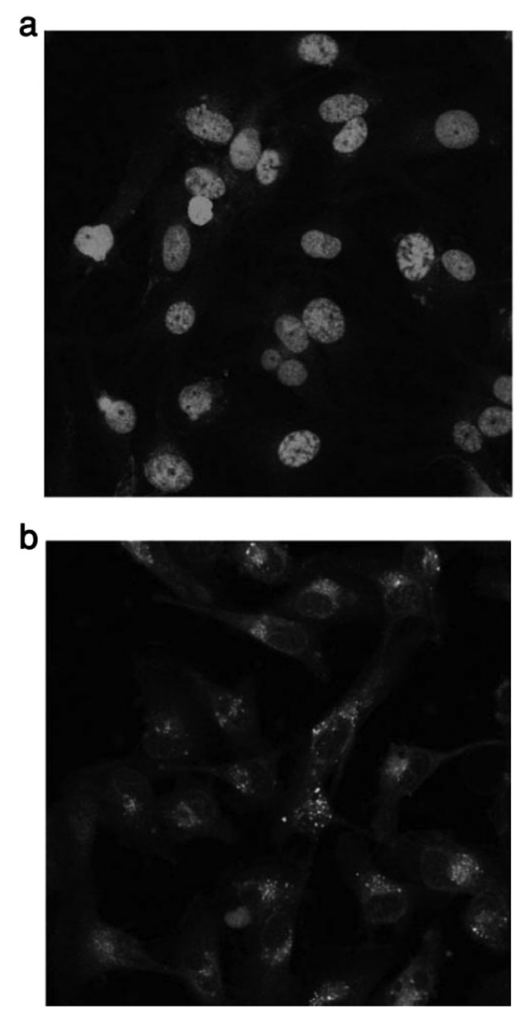

Fig. 21 Laser scanning confocal microscopy images of (a) DOX and (b) DOX-loaded $\mathrm{pH}$-sensitive micelles (PEO-block-G3 polyester dendrimer of 2,2-bis(hydroxymethyl)propanoic acid units bearing cyclic acetals of 2,4,6-trimethoxybenzaldehyde) incubated with MDAMB-231 cells for 24 h. Reprinted with permission from ref. 107. Copyright (2005) American Chemical Society. peutics in mildly acidic physiological environments make the DOX-loaded PEO-block-G3 polyester (bis-MPA) dendrimer, bearing cyclic acetals of 2,4,6-trimethoxybenzaldehyde, promising for anticancer drug delivery. The somewhat lower toxicity of the micelle system was observed in comparison with free DOX. However, the potential for the selective accumulation of the micelle system in tumor tissue by the enhanced permeation and retention effect may enhance its overall therapeutic efficacy in vivo relative to free DOX. ${ }^{107}$

Cellular uptake evaluations on Raji lymphoma cells indicated that cells treated with $\mathrm{DOX}-\mathrm{PEG}^{5 \mathrm{k}}-\mathrm{CA}_{8}$ (Compound 7) and $\mathrm{DOX}-\mathrm{PEG}^{2 \mathrm{k}}-\mathrm{CA}_{4}$ micelles showed MFI (median fluorescence intensity) 1.8-fold and 1.9-fold higher than free DOX, respectively, proving efficient internalizing of drug loaded micelles in the mentioned cells. ${ }^{108}$ Similar in vitro cytotoxicities have been found for drug loaded micelles against $\mathrm{T}$ - and B-lymphoma cells as the free drug, exhibiting $\mathrm{IC}_{50}$ values of 20-50 ng ml ${ }^{-1}$ DOX. The higher value of the maximum tolerated dose (MTD) found for DOX-PEG ${ }^{2 \mathrm{k}}-\mathrm{CA}_{4}$ micelles $(15 \mathrm{mg}$ $\mathrm{kg}^{-1}$ ) compared to free DOX $\left(10 \mathrm{mg} \mathrm{kg}^{-1}\right)$ in non-tumor bearing $\mathrm{BLAB} / \mathrm{c}$ mice has been attributed to the prolonged circulation time and the controlled drug release property. ${ }^{108}$ In the case of DOX-loaded PAMAM- $b$-PDMAEMA- $b$-PNIPAM block copolymer-modified magnetic iron oxide nanoparticles (Compound 8), ${ }^{109}$ in vitro cytotoxicity studies revealed higher inhibition on Hela cells for free DOX $\left(\mathrm{IC}_{50}=0.66 \mathrm{mg} \mathrm{mL}{ }^{-1}\right)$, in comparison with the loaded DOX in the modified nanoparticles $\left(\mathrm{IC}_{50}=1.49 \mathrm{mg} \mathrm{mL}^{-1}\right)$ at the same concentrations of DOX. This result has been explained by the slow release of the drug from the drug-loaded nanoparticles. At the same time, the result confirmed that the DOX-loaded nanoparticles were beneficial for decreasing the side effects of DOX on cells. ${ }^{109}$ In vitro cytotoxicity of blank $\mathrm{Fe}_{3} \mathrm{O}_{4}$-dendritic-linear-brush-like copolymer PAMAM- $b$-PDMAEMA- $b$-PPEGMA nanoparticles on NIH 3T3 cells demonstrated cell viability of almost $100 \% .^{110}$ Also an in vitro hemolysis assay with rabbit erythrocytes confirmed the high biocompatibility of the $\mathrm{Fe}_{3} \mathrm{O}_{4}$-modified nanoparticles. In vitro cytotoxicity investigations on HeLa cells showed $\mathrm{IC}_{50}$ values of $2.72 \mu \mathrm{g} \mathrm{mL} \mathrm{m}^{-1}$ and $\sim 0.72 \mu \mathrm{g} \mathrm{mL} \mathrm{m}^{-1}$ for DOX loaded in the modified nanoparticles (Compound 9) and free DOX, respectively. This result demonstrated that dendritic-linear-brush-like structures retard the toxic effect of DOX on the cells due to the slow release of the drug from the drugloaded nanoparticles, indicating that the modified nanoparticles can delay drug release, in which a more compact brush structure would possibly result in a lower diffusion rate of drug molecules. ${ }^{110}$ This system offers the preparation of water soluble and biocompatible modified $\mathrm{Fe}_{3} \mathrm{O}_{4}$ nanoparticles for the physical encapsulation of DOX. pH-sensitive and delayed drug release was reported, which can be beneficial to further facilitate potential biomedical applications of magnetic nanoparticles. Of course, biodistribution investigations and in vivo studies are necessary. ${ }^{110}$ It has been shown that all drug loaded PEG-Gn-PCL systems (Compound 10) delivered an effective dose of DOX to the breast cancer cells, comparable to that of the free drug. ${ }^{102}$ Determined by in vitro cytotoxicity 

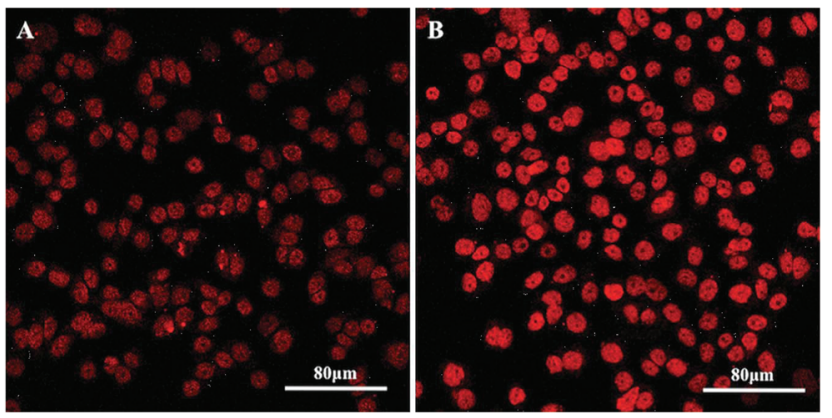

Fig. 22 Confocal microscopy of DOX in MDA-MB-468 cells. Cells were cultured in the medium with DOX-PEG5k-G1-PCL60 (containing $2 \mu \mathrm{g}$ $\mathrm{mL}^{-1} \mathrm{DOX}$ ) for $4 \mathrm{~h}$ (A) and $24 \mathrm{~h}$ (B). Both images were captured with the same parameters on confocal microscopy and adjusted to the same contrast level and brightness. Reprinted with permission from ref. 102. Copyright (2011) Wiley Periodicals, Inc.

studies, cell viability was decreased to $60-80 \%$ for MDA-MB-231 cells at $1 \mu \mathrm{g} \mathrm{mL} \mathrm{m}^{-1}$ concentration of loaded DOX, while at lower concentrations than $1 \mu \mathrm{g} \mathrm{mL} \mathrm{L}^{-1}$ the cell viability was $60 \%$ for MDA-MB-468 cells. The higher toxicity of free and loaded DOX on MDA-MB-468 cells contributes to the greater sensitivity of MDA-MB-468 cells to DOX. As proved by confocal imaging, the intensity of DOX fluorescence, released from DOX-loaded micelles, increased with time, and the fluorescence became more concentrated in the cell nuclei at the later time-point (Fig. 22).

According to flow cytometry investigations, in spite of the lower cellular uptake of DOX from the carrier-loaded DOX, the level of apoptosis of cells was comparable for free DOX and carrier-loaded DOX, confirming the PEG-Gn-PCL system as a promising anticancer drug carrier. ${ }^{102}$ Biocompatibility of empty micelles, good drug loading efficacy and efficient killing of the breast cancer cells, in spite of the lower cellular uptake, are significant advantages of the DOX loaded PEG-Gn-PCL system. However, to reduce the release rates and potentially the observed aggregation of the drug-loaded micelles, a longer PEG component is most likely necessary. Pharmacokinetics and other in vivo investigations are also necessary. ${ }^{102}$

In vitro cytotoxicity assays on the murine colon adenocarcinoma tumor C26 line demonstrated higher anticancer effects for DOX/PG-PEG-PG/MWCNTs (Compound 11), in comparison with free DOX in equal concentrations. With respect to this point that equal concentrations of free DOX and the DOX/PG-PEG-PG/MWCNT drug delivery system mean a much lower concentration of DOX in the latter case, the toxicity of DOX molecules loaded inside LLNs against cancer cells is much higher than those shown in Fig. $23 .{ }^{125}$ Hydrophilic dendritic polymers not only raise the functionality, biocompatibility and water solubility of CNTs but also change their conformations from a linear to a packed state. Changes in the conformation of the CNTs upon noncovalent interactions with PG-PEG-PG ABA type linear-dendritic copolymer led to liposome-like nanocapsules (LLNs). Since one of the proposed

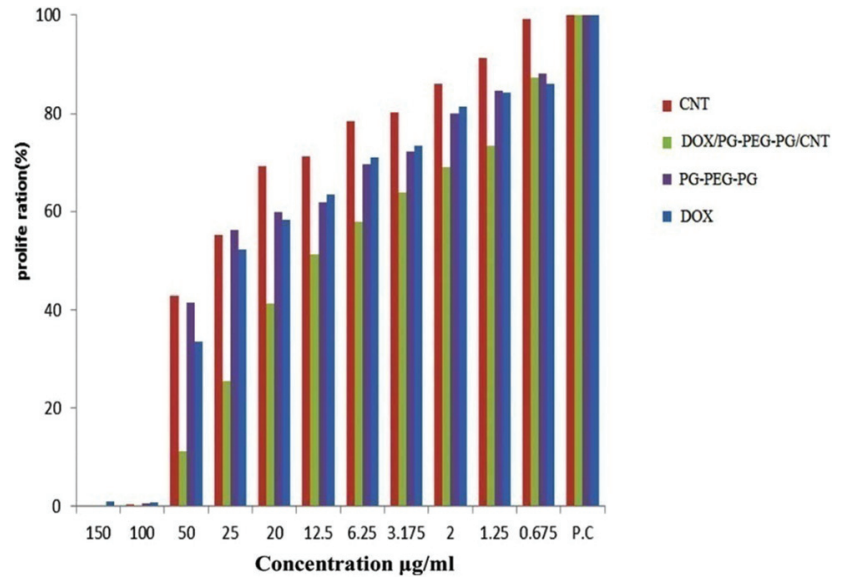

Fig. 23 The MTT assay results for opened MWCNT, PG-PEG-PG linear-dendritic copolymer, DOX/PG-PEG-PG/MWCNT drug delivery system and free DOX incubated with murine colon adenocarcinoma tumor C26 line. Reprinted with permission from ref. 125. Copyright (2012) The Royal Society of Chemistry.

reasons for the carcinogenicity of carbon nanotubes is their length and rigid structure, flexible liposome-like nanocapsules prepared by this strategy could be safer and far from the asbestos-like physicochemical properties of CNTs and therefore their potential health hazards. Avoiding these health hazards, it is possible to develop CNTs for biomedical applications. ${ }^{125}$

By in vitro cytotoxicity tests conducted on mouse tissue connective fibroblast adhesive cell line (L929), it was found that in low concentrations the toxicity of DOX-loaded $\mathrm{Fe}_{3} \mathrm{O}_{4}$-MWCNTs/ PAMAM-PEG-PAMAM hybrid nanomaterials (Compound 12) was much higher than other systems and even free DOX, indicating the critical role of the carbon nanotube in transferring hybrid nanomaterial drug delivery systems, and therefore loaded DOX, from the cell membrane. ${ }^{32}$ Since PEG improves the processability, water solubility and long blood circulation of CNTs through non-covalent interactions, supramolecular interactions between linear-dendritic PAMAM-PEG-PAMAM copolymers and CNTs lead to water soluble and high functional hybrid nanomaterials. Additionally, high loading capacity, higher toxicity compared to free DOX, and good superparamagnetic behavior make DOX/ $\gamma$ - $-\mathrm{Fe}_{3} \mathrm{O}_{4}$-MWCNTs/ PAMAM-PEG-PAMAM a promising material for anticancer drug delivery. ${ }^{32}$

By in vitro cytotoxicity assays in the breast cancer cells (MDA-MB-231), the PEG-block-bis-MPA polyesters (G4)-(Bz) have been found to be nontoxic. ${ }^{94}$ Also a reduced cell viability of $55 \%$ has been determined for DOX-PEG-(G4)-(Bz) $)_{8}$ (Compound 13) containing $10 \mu \mathrm{g} \mathrm{ml}^{-1} \mathrm{DOX}$, in comparison with $73 \%$ cell viability obtained at the same concentration of free DOX, showing that DOX-loaded micelles delivered the therapeutic effect with high efficacy. ${ }^{129}$ In summary, DOX encapsulated PEG-block-bis-MPA polyesters (G4)-(Bz) showed some advantages, such as nontoxicity of the linear-dendritic carrier and the higher cytotoxic effect against MDA-MB-231 cancer 
cells in comparison with free DOX. However, higher molecular weights of PEG are necessary to provide sufficient stealth or steric repulsion during DOX loading and to avoid aggregation. Additionally, strategies for achieving better controlled release kinetics should be noticed. Pharmacokinetics studies and in vivo tests can help this system develop for cancer therapy. ${ }^{129}$

By flow cytometry profiles it has been proved that the DOXloaded PEO5K-PAMAM (D3)-diazonaphthoquinone (DNQ) micelles (Compound 14) could quickly enter into HeLa cells in a time-independent manner compared with free DOX (Fig. 24). ${ }^{132}$ This phenomenon has been attributed to the nanomedicines of $<200 \mathrm{~nm}$ internalization by HeLa cells in an endocytosis process, compared with a diffusion process for the cellular uptake of free DOX. ${ }^{135}$ In vitro cytotoxicity studies evaluated in HeLa cells at $6 \mu \mathrm{g} \mathrm{mL} \mathrm{m}^{-1}$ DOX dosage showed the cell viability of $\sim 65 \%$ and $\sim 18 \%$ for the DOX-loaded PEO5KD3DNQ micelles and free DOX, respectively. This lower cytotoxicity of DOX-loaded micelles corresponds to their sustained drug-release behaviour. Interestingly, the cell viability for the DOX-loaded micelles decreased to $\sim 35 \%$ after 30 min of $808 \mathrm{~nm}$ irradiation, exhibiting a NIR-triggered cytotoxicity. ${ }^{132}$ Collectively, DOX-loaded PEO5K-PAMAM (D3)-diazonaphthoquinone (DNQ) micelles demonstrated some interesting advantages, including the stability of drug-free micelles in PBS at $37{ }^{\circ} \mathrm{C}$ and $\mathrm{pH}=7.4$, tuned release of DOX by NIR light irradiation, quick entrance into HeLa cells compared to free DOX, DOX release inside the cells, and then killing the cells in a NIR-triggered manner. Disrupting the micelles by $808 \mathrm{~nm}$ NIR irradiation may be a challenge for this system, however biodistribution studies and in vivo tests can develop this promising anticancer system. ${ }^{132}$

5.1.3. In vivo evaluations. Biodistribution studies showed 2.0-fold and 2.2-fold higher drug uptake for DOX-PEG ${ }^{5 \mathrm{k}}-\mathrm{CA}_{8}$ and $\mathrm{DOX}-\mathrm{PEG}^{2 \mathrm{k}}-\mathrm{CA}_{4}$ micelles compared to that for free DOX in the tumor tissue of treated mice. ${ }^{108}$ This enhanced accumulation corresponded to the prolonged circulation and the EPR effect in linear-dendritic micelle formulations (Compound 7). Compared to free DOX, significantly reduced drug distribution in the heart has been achieved by both DOX-PEG-CA formulations. However, relatively higher uptake in the liver and
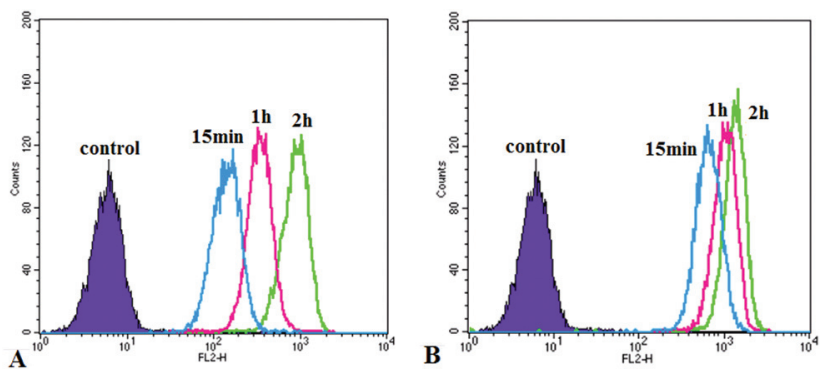

Fig. 24 Flow cytometry histogram profiles of HeLa cells incubated with free DOX (A) and DOX-loaded micelles of PEO5K-PAMAM (D3) DNQ (B) for different time intervals. Reprinted with permission from ref. 132. Copyright (2013) The Royal Society of Chemistry. spleen has been exhibited for DOX-micellar systems caused by nonspecific elimination of micellar NPs via the reticuloendothelial system (RES), such as macrophage in the liver and spleen. Better inhibition of tumor growth was shown for DOX$\mathrm{PEG}^{2 \mathrm{k}}-\mathrm{CA}_{4}$ micelles because of their longer retention time and slower drug release rate. By day 28 post-injection, the relative tumor volume (RTV) of 7.7 and 6.8 was achieved for Raji lymphoma bearing mice treated with $\mathrm{DOX}-\mathrm{PEG}^{5 \mathrm{k}}-\mathrm{CA}_{8}$ and DOX$\mathrm{PEG}^{2 \mathrm{k}}-\mathrm{CA}_{4}$, respectively. This value was 9.9 for free DOX treated mice. ${ }^{108}$ Besides stability under physiological conditions, DOX encapsulated $\mathrm{PEG}^{\mathrm{mk}}-\mathrm{CA}_{n}$ micelles offered some significant advantages in comparison to free DOX, such as higher in vitro cellular uptake, higher maximum tolerated dose, increased retention time in the blood, higher uptake in tumor tissue in vivo, higher antitumor efficacy in vivo, and reduced drug distribution in the heart. These all make $\mathrm{PEG}^{\mathrm{mk}}$ $\mathrm{CA}_{n}$ micelles attractive for cancer therapy (Table 3). ${ }^{108}$

\subsection{Doxorubicin-conjugated linear-dendritic block copolymers}

5.2.1. Physicochemical properties. Padilla De Jesús et $a l .{ }^{136}$ have designed a soluble carrier for DOX consisting of a 3-arm poly(ethylene oxide) (PEO) and three [G-2] dendritic polyester with 2,2-bis(hydroxymethyl)propanoic acid monomer units (Compound 15), providing the multivalency necessary for drug attachment (Fig. 25). DOX has been covalently linked to the linear-dendritic structure via an acid-labile hydrazone, which can remain stable under physiological conditions, and can be cleaved in the vicinity of a tumor prior to being internalized by the cancer cell because of the more acidic environment around the tumor tissue. ${ }^{136}$

Drug release studies indicated that a $100 \%$ release would be achieved after $10 \mathrm{~min}, 3 \mathrm{~h}, 26.5 \mathrm{~h}$, and 10 days for $\mathrm{pH}$ 2.5, 4.5, 5.5 , and 6.5 , respectively. This confirms the suitability of the hydrazone linkage for a $\mathrm{pH}$-dependent release that is compatible with conditions found in tumors. ${ }^{136}$

Gillies and Fréchet also reported interesting polyester dendrimer-PEO bow-tie hybrids consisting of 2,2-bis(hydroxymethyl) propionic acid based polyester dendrimers and PEO in which PEO was linked to the dendritic scaffold via carbamate bonds. ${ }^{137,138}$

Later, the $[\mathrm{G}-3]-\left(\mathrm{PEO}_{5 \mathrm{k}}\right)_{8}-[\mathrm{G}-4]-(\mathrm{OH})_{16}$ bow-tie structure (molecular mass $\sim 45 \mathrm{kDa}$ ) was utilized to provide the dendrimer-DOX conjugates by coupling hydrazide linkers to the hydroxyl groups of the bow tie (Compound 16), followed by hydrazone formation with DOX hydrochloride and subsequent chromatographic separation from free DOX. DOX loading was consistently found to be $8-10 \mathrm{wt} \%$ for different batches. ${ }^{139,140}$ Notably, the bow-tie DOX conjugate was readily dissolved in water at DOX concentrations as high as $6 \mathrm{mg} \mathrm{ml}^{-1}(\sim 60 \mathrm{mg}$ $\mathrm{ml}^{-1}$ polymer), indicating that the PEO arms of the bow-tie dendrimer can shield the hydrophobic drug moieties at the core of the molecule, perhaps in a structure similar to that of a unimolecular micelle. A volume average hydrodynamic diameter of $8 \mathrm{~nm}$ for the conjugate was determined using dynamic light scattering, indicating that intermolecular 
Table 3 Doxorubicin-encapsulated linear-dendritic block copolymers

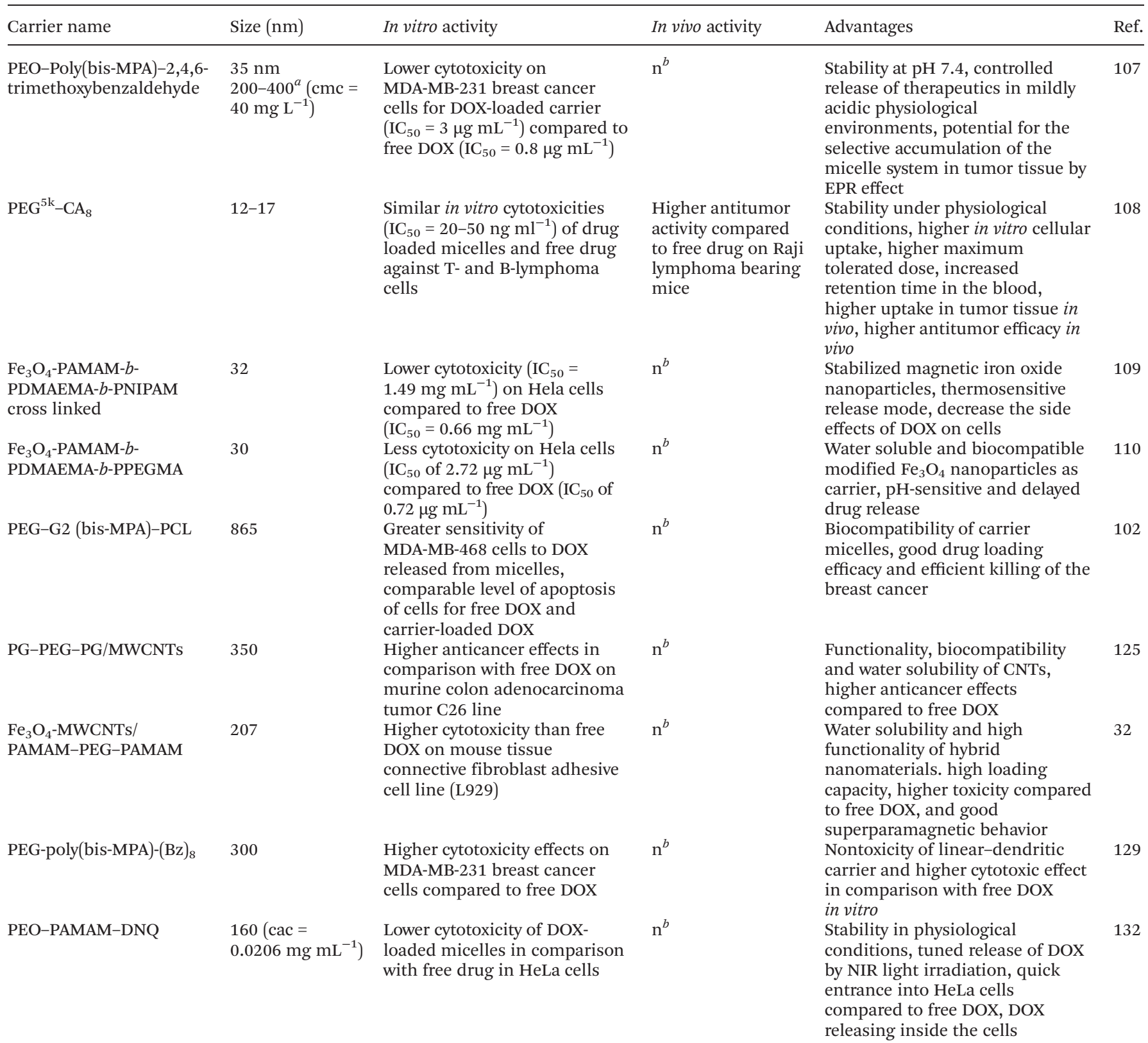

${ }^{a}$ A small fraction of aggregates. ${ }^{b}$ n: not reported.

aggregation did not occur. ${ }^{139}$ The release of the drug from the DOX-conjugated bow-tie polymer at $\mathrm{pH} 5.0$ and 7.4 buffers at $37{ }^{\circ} \mathrm{C}$ was monitored chromatographically. At $\mathrm{pH} 5$, the drug was released from the dendrimer rapidly, and the concentration of free doxorubicin in solution increased steadily with $t_{1 / 2}=6 \pm 1 \mathrm{~h}$, reaching $100 \%$ release within $48 \mathrm{~h}$. Only a small amount $(<10 \%)$ of the same compound was released after $48 \mathrm{~h}$ at pH 7.4 (Fig. 26). ${ }^{139,140}$

Huang et al. ${ }^{141}$ have reported the liver-targeting potential of polymeric prodrug of doxorubicin bearing Galactose conjugated linear dendritic block copolymers. Galactose as the targeting ligand has been conjugated to linear PEG, and DOX has been coupled to the PAMAM dendritic section via an acid-labile hydrazone linker to produce the Gal-PEG- $b$-PAMAM-DOX ${ }_{n}$ drug delivery system (Compound 17) (Fig. 27). It has been demonstrated that the galactosylated drug carrier could reach hepatocytes via receptor-mediated active targeting, due to the high affinity of asialo-glycoprotein (ASGP) receptor to galactosyl residues. ${ }^{142}$

Determined by ${ }^{1} \mathrm{HNMR}$, the molar ratio of DOX to copolymer was $5.5: 1$, which was lower than the theoretical value $(8: 1)$, because of the steric hindrance that leads to the lower drug loading. 


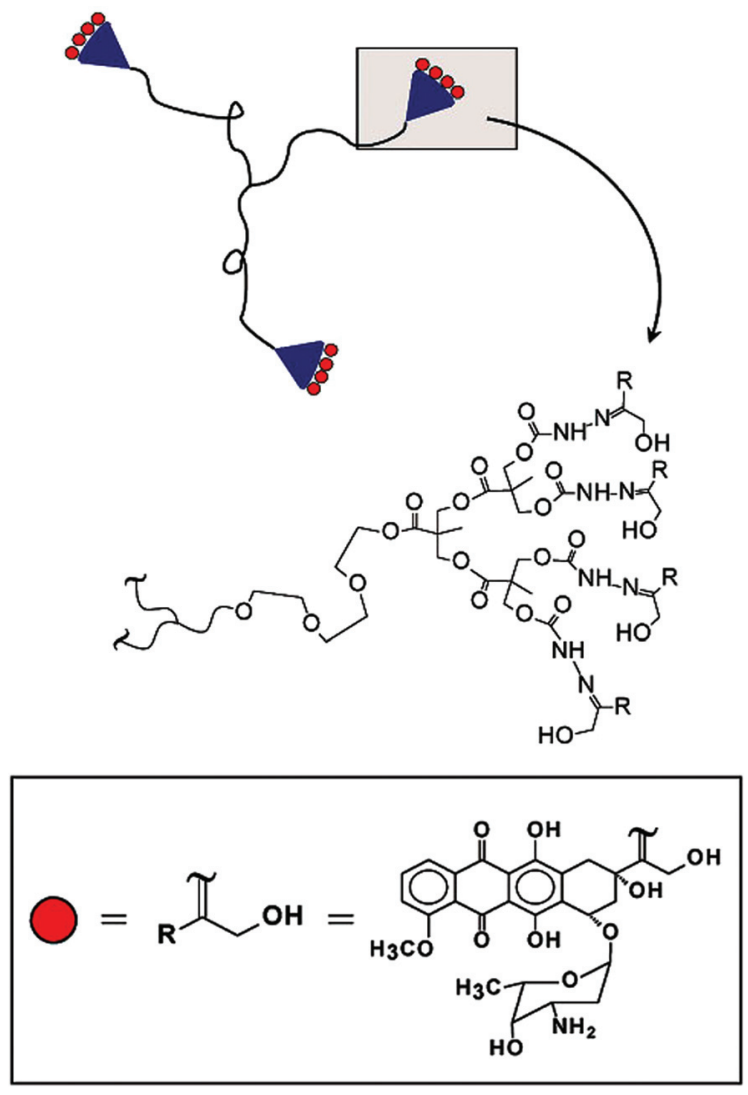

Fig. 25 Polymer drug conjugate consisting of 3-arm PEO-polyester dendrimer and doxorubicin using a hydrazone covalent bond as a linker (Compound 15). Reprinted with permission from ref. 136. Copyright (2002) American Chemical Society.

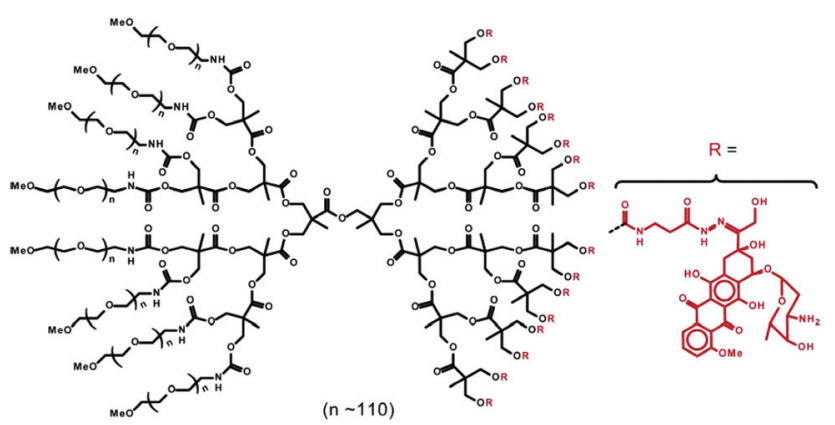

Fig. 26 [G-3]-(PEO $\left.{ }_{5 k}\right)_{8}$-[G-4]- $(\mathrm{OH})_{16}$-doxorubicin conjugates (Compound 16). Reprinted with permission from ref. 140. Copyright (2006) American Chemical Society.

The acid-sensitive degradation of the hydrazone linker between DOX and the Gal-PEG- $b$-PAMAM carrier caused a $\mathrm{pH}$ triggered drug release profile. It has been determined that 14 and $32 \%$ of DOX was released at $\mathrm{pH}$ of 8.0 and 7.4 , respectively, in $30 \mathrm{~h}$. On the other hand, at $\mathrm{pH}=5.6,97 \%$ of DOX release was observed after $15 \mathrm{~h}$. This indicates the stable circulation of the polymeric drug in the bloodstream $(\mathrm{pH}=7.4)$,

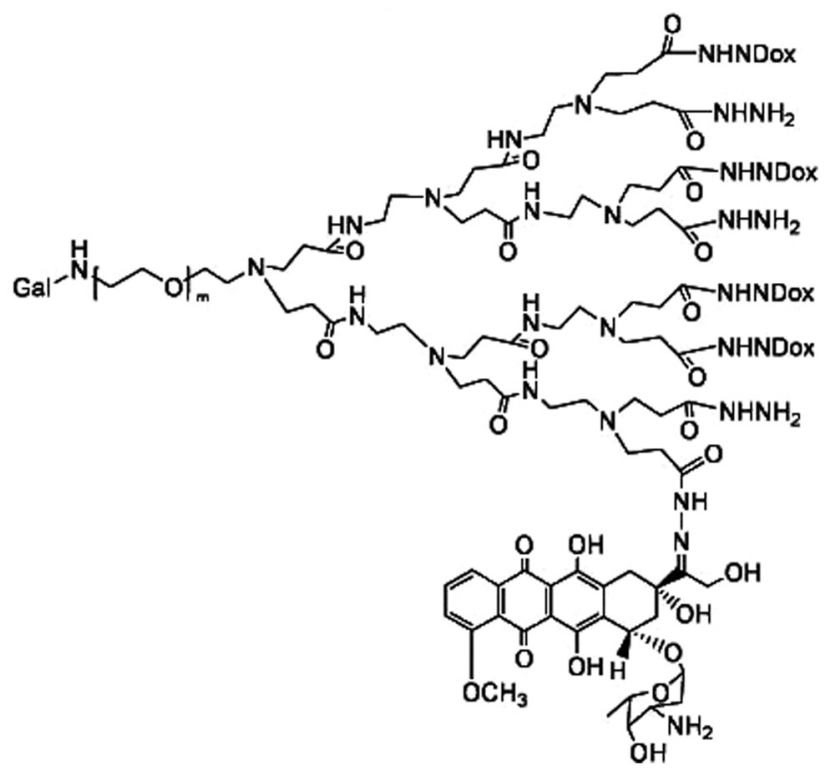

Fig. 27 Gal-PEG-b-PAMAM-DOX ${ }_{n}$ prodrug (Compound 17). Reprinted with permission from ref. 141. Copyright (2010) Society of Chemical Industry.

and triggered drug release in endosomes and lysosomes $(\mathrm{pH}=$ 5.6-6.5) of cancer cells. ${ }^{141}$

In a recent study, She et al. have demonstrated the use of mPEGylated peptide Dendron-DOX conjugate (Compound 18) as a pH-stimulated drug delivery system for breast tumor therapy. ${ }^{143}$ In their study, the tail of the L-lysine dendron has been functionalized with two alkynyl groups. Then, mPEG ( $2 \mathrm{kDa}$ ) with an azido group at one end has been covalently linked to the peptide dendron by $\mathrm{Cu}^{\mathrm{I}}$-catalyzed azide-alkyne cycloaddition (CuAAC). DOX has been conjugated to the dendron through a pH-sensitive hydrazone bond, resulting in a compact nanoparticle, via self-assembly governed by Dendron-DOX itself, as shown in Fig. 28.

UV-vis spectrophotometry indicated the presence of 2 DOX molecules for each mPEGylated peptide Dendron-doxorubicin (14 wt\%). The mPEGylated peptide Dendron-DOX conjugate

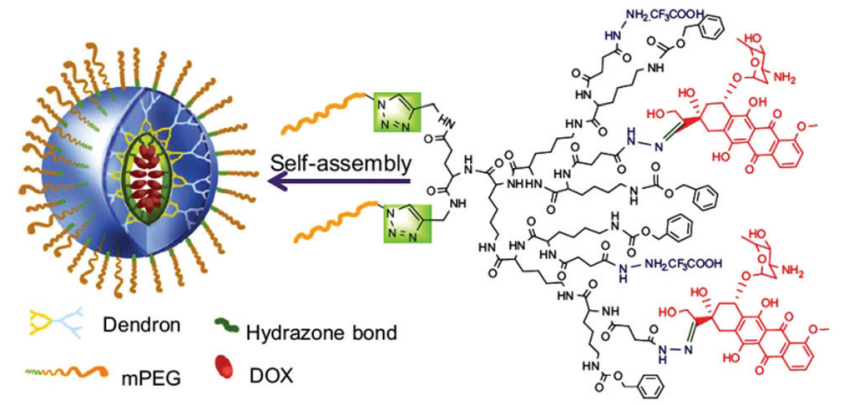

Fig. 28 Structures of mPEGylated peptide dendron, and the illustration of the dendron-DOX conjugate based nanoparticle (Compound 18). Reprinted with permission from ref. 143. Copyright (2012) Elsevier Ltd. 
aggregated to a nanosized particle in water $(\mathrm{pH}=7.4)$, displaying average hydrodynamic sizes around $220 \mathrm{~nm} .{ }^{143}$ Generally, producing drive force segments is needed to introduce to the PEGylation and functionalization of the dendron for selfassembly. ${ }^{144}$ For this designed dendron, the self-assembly behavior was mediated by the mPEGylated peptide DendronDOX itself. The primary driving force responsible for the selfassembly behavior is the minimization of the interfacial energy, governed by the balance between the hydrophilic interaction of the linear mPEG and the hydrophobic interaction of the Dendron-DOX block. ${ }^{144}$ Secondly, the driving forces governing the self-assembly of the prepared mPEGylated peptide Dendron-DOX, such as $\pi-\pi$ stacking, dipole interactions, $\mathrm{H}$ bonding and the pre-organized branched architecture of the dendritic block, should also be considered, since the DOX is composed of multiple domains of different chemical compositions, e.g., hydrophobic, aliphatic and aromatic. ${ }^{145}$ At predetermined time points, higher release amounts (80\%) at $\mathrm{pH} 5$, in comparison with release amounts at pH 7.4 (20\%), revealed the $\mathrm{pH}$-sensitive mode of drug release for mPEGylated peptide Dendron-doxorubicin nanoparticles. This accelerated release has been attributed to the cleavage of hydrazone linkers at lower $\mathrm{pH}$ values. ${ }^{143}$

In another study She et al. have also used $\mathrm{Cu}^{\mathrm{I}}$-catalyzed azide-alkyne cycloaddition (CuAAC) click chemistry to covalently attaching the L-lysine peptide dendron to heparin, resulting in water-soluble dendronized heparin. ${ }^{146}$ Then, DOX has been conjugated to the surface of the dendron through a $\mathrm{pH}$-sensitive hydrazone bond, resulting in a compact nanoparticle via self-assembly in water $(\mathrm{pH}=7.4)$, displaying average hydrodynamic sizes around $90 \mathrm{~nm}$ and PDI of 0.140 (DOX content $9 \mathrm{wt} \%$ ) (Compound 19) (Fig. 29).

Self-assembly behavior was mediated by the dendronized heparin-DOX conjugate itself. As mentioned before, ${ }^{146}$ the primary driving force responsible for the self-assembly behavior is the minimization of the interfacial energy governed by the balance between the hydrophilic interaction of the linear polymer and the hydrophobic interaction of the dendronized heparin-DOX block. Secondly, the driving forces governing the

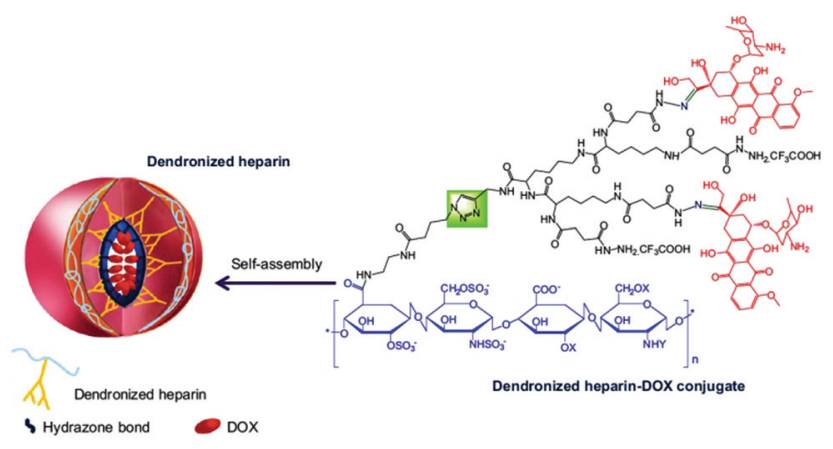

Fig. 29 Illustration of a dendronized heparin-DOX conjugate based nanoparticle (Compound 19). Reprinted with permission from ref. 146. Copyright (2012) Elsevier Ltd. self-assembly of prepared dendronized heparin-DOX, such as $\pi-\pi$ stacking, dipole interactions, $\mathrm{H}$-bonding and the preorganized branched architecture should also be considered, since the DOX is composed of multiple domains of different chemical compositions, e.g., hydrophobic, aliphatic and aromatic. Based on drug release profiles, showing $20 \%$ DOX release at $\mathrm{pH} 7.4$ after $56 \mathrm{~h}$ incubation, it was concluded that the dendronized heparin-DOX system was stable in the circulation system ( $\mathrm{pH}$ 7.4). In contrast, cleavage of the acid-labile hydrazone bonds of DOX-conjugated nanoparticles accelerated the release of drug at pH 5.0 (>80\%), indicating the ability of nanoparticles to release the DOX in the acidic endosomes and/or lysosomes with $\mathrm{pH}$ ranging from 4.0-6.0. ${ }^{146}$

In a research work reported by Zhang et al., ${ }^{147}$ a co-delivery strategy for anti-cancer treatment has been employed, utilizing 10-hydroxycamptothecin (HCPT) encapsulated MPEG- $b$ PAMAM-DOX amphiphilic linear-dendritic prodrug. In the preparation route, MPEG- $b$-PAMAM G2.5 has been hydrazinolysized to MPEG- $b$-PAMAM G3X by hydrazine hydrate. Then, DOX as the hydrophobic part of the amphiphilic copolymer has been conjugated to PAMAM via an acid-labile hydrazone linkage by reacting with the keto groups of DOX. UV absorbance studies gave a DOX content of $52.9 \mathrm{wt} \%$ for MPEG2000$b$-PAMAM-DOX and $31.0 \mathrm{wt} \%$ for MPEG5000- $b$-PAMAM-DOX. HCPT loading into DOX-conjugated nanoparticles has been carried out by a solvent displacement method with $\mathrm{pH}$ adjusted to 6.5 , in which the hydrazone bond was stable while HCPT could maintain its lactone form. ${ }^{148,149}$ HCPT content has been determined to be 19.2 and $21.6 \mathrm{wt} \%$ for MPEG2000$b$-PAMAM-DOX and MPEG5000- $b$-PAMAM-DOX (Compound 20) nanoparticles, respectively. The nanoparticles were of uniform size and spherical shape. The radii of nanoparticles formed by MPEG2000- $b$-PAMAM-DOX and MPEG5000- $b$ PAMAM-DOX were about 50 and $60 \mathrm{~nm}$, respectively. As a hydrophobic molecule, HCPT was wrapped in the core of the nanoparticles, which were formed by the self-assembly of MPEG- $b$-PAMAM-DOX prodrugs. The radii of the HCPT loaded nanoparticles formed by MPEG2000- $b$-PAMAM-DOX and MPEG5000- $b$-PAMAM-DOX were $88 \mathrm{~nm}$ and $122 \mathrm{~nm}$, respectively. The increase in size of the nanoparticles after HCPT loading indicates the successful incorporation of HCPT in the hydrophobic core. ${ }^{147} \mathrm{~A}$ pH-dependent release mode has been demonstrated for both drugs released from HCPT loaded MPEG- $b$-PAMAM-DOX. There has been no initial DOX burst release from DOX-conjugated nanoparticles because of the chemical combination of DOX to the copolymer. The release of DOX molecules was negligible at $\mathrm{pH} 7.4$, indicating the stability of a hydrazone bond at this $\mathrm{pH}$. Lowering the $\mathrm{pH}$ to 5.5 and 4.5 caused an increase in DOX release of about $30 \%$ and $60 \%$ in $48 \mathrm{~h}$, respectively. Also faster release of HCPT has been demonstrated in $\mathrm{pH} 4.5$ compared to $\mathrm{pH} 5.5$, attributed to the faster cleavage of DOX and faster disassembly of the selfassembled nanoparticles at lower $\mathrm{pH}$ (Fig. 30). ${ }^{147}$

Another $\mathrm{pH}$-responsive prodrug formulation based on linear-dendritic MPEG- $b$-PAMAM has been recently reported by Zhang et $a .^{78}$ Firstly, linear-dendritic MPEG- $b$-PAMAM was 


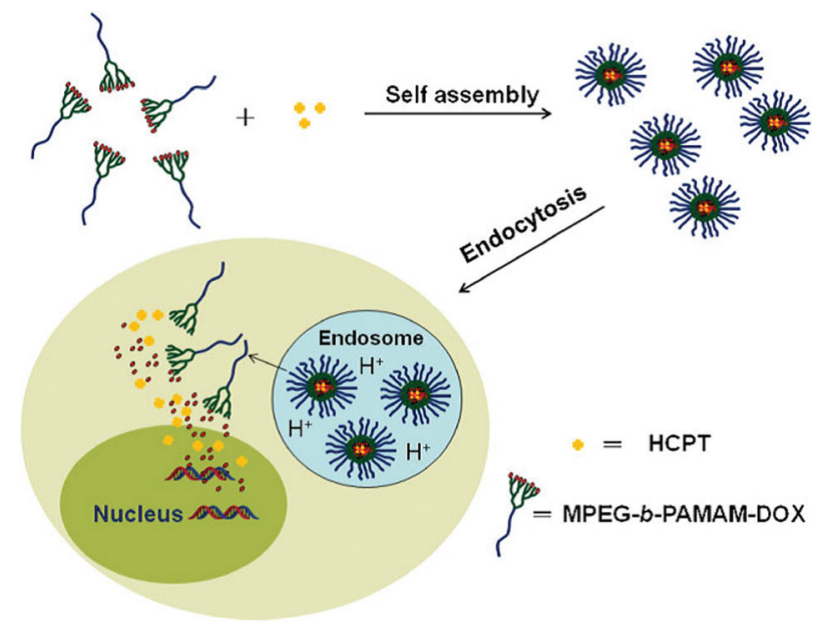

Fig. 30 Illustration of MPEG-b-PAMAM block copolymer conjugated with DOX and its self-assembled HCPT loaded nanoparticles for the $\mathrm{pH}$ responsive intracellular release (Compound 20). Reprinted with permission from ref. 147. Copyright (2013) Wiley-VCH Verlag GmbH \& Co. KGaA, Weinheim.

modified with lipoic acid (LA) and then doxorubicin was conjugated to the modified structure by an acid-labile hydrazone bond, resulting in amphiphilic structures that could be selfassembled to the nanosized micelles (Compound 21) (Fig. 31). Due to the significant glutathione (GSH) concentration difference between the extracellular milieu $(2-20 \mathrm{mM})$ and the cytoplasm $(2-10 \mathrm{mM})$, the reduction responsive cross-linked micelles are attracting more and more attention. Therefore, the obtained MPEG- $b$-PAMAM-LA/DOX micelles have been cross-linked by disulfide bonding through introducing $10 \mathrm{~mol} \%$

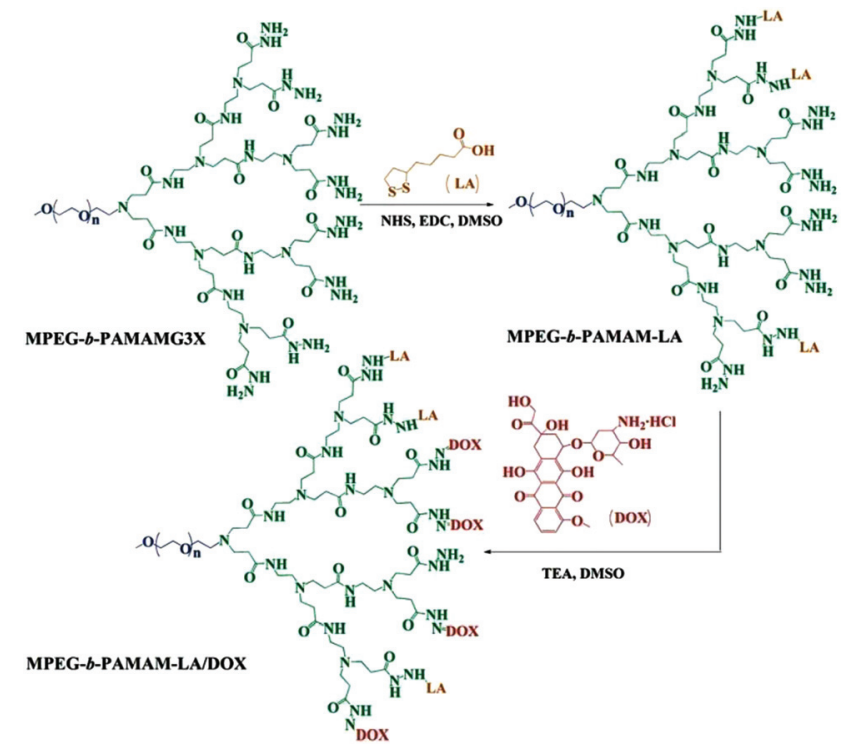

Fig. 31 Synthesis pathway of MPEG-b-PAMAM-LA/DOX (Compound 21). Reprinted with permission from ref. 78. Copyright (2014) the Royal Society of Chemistry.
DTT relative to the lipoyl units in borate buffer ( $\mathrm{pH} 8.5)$. With the MPEG segment as the hydrophilic moiety and LA and DOX as the hydrophobic moieties, the amphiphilic prodrug self-assembled into spherically shaped micelles. The particle size of the cross-linked prodrug particles was $\sim 140 \mathrm{~nm}$. The stability of the cross-linked MPEG- $b$-PAMAM-LA/DOX NPs was evaluated in $20 \mathrm{mM}$ PB solution for different time intervals. After standing for 4 days, 8 days and 16 days, the particle size of the NPs only slightly changed from the original $144 \mathrm{~nm}$ to $161 \mathrm{~nm}$ after 16 days of incubation, also suggesting the good stability of the cross-linked NPs. The change in the particle size in response to $10 \mathrm{mM} \mathrm{GSH}$ was monitored over time in $20 \mathrm{mM}$ PB solution, to investigate whether cross-linked NPs can be de-cross-linked in a reductive environment. It was shown that the addition of GSH led to a dramatic increase and wide distribution of the particle size, implying the cleavage of disulfide bonds by reduced GSH. The DOX loading content of cross-linked MPEG- $b$-PAMAM-LA/DOX micelles has been determined to be $25.6 \mathrm{wt} \%$. The high drug loading content has been ascribed to the multiple amine groups on the PAMAM backbone. $^{78}$

A pH-dependent release for DOX from core-cross-linked MPEG- $b$-PAMAM-LA/DOX nanoparticles has been demonstrated. At $\mathrm{pH} 7.4$, only about $6 \%$ of the loaded DOX was released in $72 \mathrm{~h}$, while about $35 \%$ of DOX was released at $\mathrm{pH}$ 5.5. These results indicate that the hydrazone bond is stable at the physiological $\mathrm{pH}$ of 7.4, but would be cleaved at the endosomal $\mathrm{pH}$ of 5.5. Also reduction-sensitive release behavior has been found for cross-linked MPEG-b-PAMAM-LA/DOX. At the same $\mathrm{pH}$ value, higher DOX release has been achieved with a higher GSH concentration. This has been attributed to the cleavage of the disulfide bonds by GSH, leading to de-crosslinking of the nanoparticles and the rapid release of DOX. ${ }^{78}$

5.2.2. In vitro evaluations. Based on in vitro cytotoxicity of the drug-polymer conjugate, evaluated on three cell lines B16F10, MDA-MB-435, and MDA-MB-231, the free drug was more potent than the DOX-conjugated 3-arm (PEO) star-three [G2] polyester dendritic block with bis-MPA units (Compound 15); 6-fold in the B16F10 cells, 50-fold in the MDA-MB-231, and 9-fold in the MDA-MB-435 cells. Cell uptake of the polymer-drug conjugate (monitored via fluorescence confocal microscopy) proved the cell uptake of the conjugate by endocytosis, through fluorescence observation in the cytosol. On the other hand, both the cytoplasm and the nuclei were highly fluorescent after exposure to free doxorubicin. ${ }^{136}$

In spite of substantial intratumoral concentrations of polymer and drug, attempts at chemotherapy utilizing the doxorubicin functionalized 3-armed (PEO) star-three [G2] polyester bis-MPA dendrimer in murine tumor models (B16F10) were largely unsuccessful. ${ }^{140}$ This was attributed to hydrazone carboxylate linkages, which were utilized in this system to attach a doxorubicin topolymeric structure (Fig. 25). Lee et al. ${ }^{140}$ investigated the hydrolysis kinetics of hydrazone carboxylate linked doxorubicin and proposed intramolecular nucleophilic attack of the C-14 hydroxyl of doxorubicin on the carbonyl group of the hydrazone carboxylate linker (Fig. 32). 


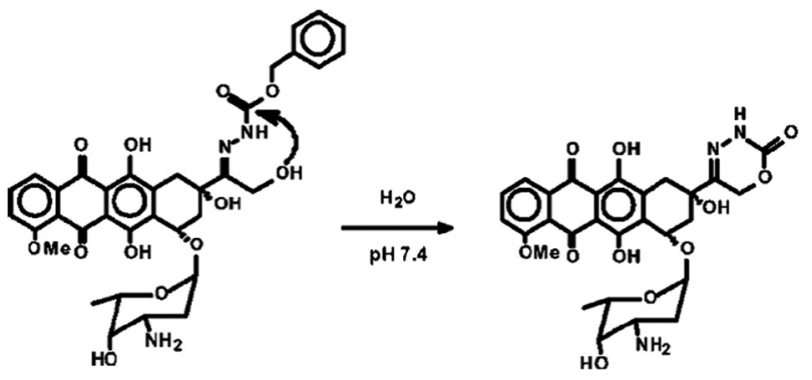

Fig. 32 Intramolecular cyclization in a hydrazone carboxylate linked doxorubicin system. Reprinted with permission from ref. 140. Copyright (2006) American Chemical Society.

The proposed intramolecular cyclization reaction involving the doxorubicin C-14 hydroxyl and the carboxylate-substituted hydrazone rationalizes the seemingly anomalous hydrolysis kinetics seen for hydrazone carboxylate linked doxorubicin, and provides a possible explanation for the poor antitumor activity exhibited by polymer-doxorubicin conjugates utilizing this specific type of linkage. ${ }^{140}$

In vitro cytotoxicity studies of polyester dendrimer-PEO bow-tie hybrids, with a range of MWs $20-100 \mathrm{KDa}$ as well as low and high degrees of branching, on MDA-MB-231 cancer cells, showed no significant toxicity up to $10 \mathrm{mg} \mathrm{mL}^{-1}$, the highest concentration evaluated, with cell viabilities exceeding $85 \%$ relative to controls at all concentrations. ${ }^{138}$ The cytotoxic activity of DOX-conjugated bow-tie polyester dendrimer-PEO (Compound 16) on C-26 cells was found to be considerably less than that for the free DOX on an equimolar basis $\left(\mathrm{IC}_{50}\right.$, DOX $=0.08 \pm 0.02 \mu \mathrm{g} \mathrm{ml}{ }^{-1} ; \mathrm{IC}_{50}$, hydrazone bow-tie DOX = $1.4 \pm 0.2 \mu \mathrm{g} \mathrm{ml} \mathrm{m}^{-1}$ ). This result was attributed to the slower rate of cellular uptake for the dendrimers when compared with the free drug and to the gradual release of free drug from the polymers, due to hydrolysis of the linkers and the polyester dendrimer backbone. ${ }^{139}$

By in vitro cytotoxicity studies against Bel-7402 cells, the cell viability has been determined as $11 \%$, 60\% and 50\% in cells treated with free DOX, non-targeting PEG- $b$-PAMAM-DOX ${ }_{m}$, and targeting Gal-PEG- $b$-PAMAM-DOX ${ }_{n}$ (Compound 17), respectively, at DOX concentration of $40 \mu \mathrm{g} \mathrm{mL} \mathrm{m}^{-1} \cdot{ }^{141}$ Compared to free DOX, decreased cytotoxicity of the polymeric prodrugs has been attributed to their gradual drug release profiles. Also higher cytotoxicity of galactose conjugated prodrug in comparison with the non-targeting one has been explained by the receptor-mediated higher cell uptake of Gal-PEG- $b$-PAMAMDOX $_{n}$. ASGP receptors in Bel-7402 and galactosyl residue in the prodrug are responsible for the increased intracellular drug concentration. ${ }^{141}$

Lower in vitro cytotoxicity, evaluated on mouse breast cancer cell line (4T1), was seen for mPEGylated peptide Dendron-DOX conjugate nanoparticles (Compound 18) $\left(\mathrm{IC}_{50}=\right.$ $\left.151 \mathrm{ng} \mathrm{mL} \mathrm{mL}^{-1}\right)$ compared with free $\mathrm{DOX}\left(\mathrm{IC}_{50}=25.9 \mathrm{ng}\right.$ $\left.\mathrm{mL}^{-1}\right) .{ }^{143}$ This has been explained by the amphipathic properties of DOX and the higher ability of small molecules to cross the cell membrane. Cell viability of $95 \%$ for non-drug conjugated nanoparticles indicated that the mPEGylated peptide dendron was nontoxic and DOX released from the DOX-conjugated nanoparticle in the acidic environment of endosomes was responsible for cytotoxicity. ${ }^{143}$

Dendronized heparin-DOX (Compound 19) showed $\mathrm{IC}_{50}$ of $300 \mathrm{ng} \mathrm{mL} \mathrm{m}^{-1}$ against the mouse breast cancer cell line (4T1), approximately 11-fold of free DOX with $\mathrm{IC}_{50}$ of $27 \mathrm{ng} \mathrm{mL}{ }^{-1}{ }^{146}$ This lower cytotoxicity of dendronized heparin-DOX was due to the amphipathic properties of free DOX and its ability to easily cross the cell membrane. More than $90 \%$ of $4 \mathrm{~T} 1$ cells have still been alive after the treatment with drug-free dendronized heparin, showing non-cytotoxicity of the dendronized heparin nanoparticle. So it is clear that the cytotoxicity of the nanoparticle with the drug would not be due to the dendronized heparin block, but the drug DOX, proving the release of DOX from the nanoparticle in the acidic environment of endosomes. $^{146}$

Flow cytometric analysis demonstrated that HCPT loaded MPEG- $b$-PAMAM-DOX nanoparticles (Compound 20) could be effectively taken up by MCF-7 cells. ${ }^{147}$ After $10 \mathrm{~h}$ incubation with MCF-7 cells, about $80 \%$ HCPT has been internalized by MCF-7 cells from both HCPT loaded MPEG- $b$-PAMAM-DOX nanoparticles, much higher than the internalization content of free HCPT, indicating that DOX conjugated nanoparticles increased the solubility of free HCPT and delivered HCPT efficiently to MCF-7 cells. Higher in vitro cytotoxicity has been determined for HCPT loaded MPEG- $b$-PAMAM-DOX nanoparticles compared to free DOX and free HCPT in MCF-7 and HepG2 cell lines. Moreover, the HCPT loaded MPEG- $b$-PAMAM-DOX exhibited better cytotoxicities than the physical mixtures of MPEG- $b$-PAMAM-DOX and HCPT. ${ }^{147}$ This confirmed that the DOX conjugated prodrugs could effectively encapsulate HCPT and subsequently release it in the cell, leading to enhanced drug activity and better in vitro antitumor effect for the co-delivery system. Enhanced cell apoptosis has been demonstrated for the co-delivery system. Evaluated by flow cytometry in MCF-7 cells, HCPT loaded MPEG2000- $b$-PAMAM-DOX and MPEG5000$b$-PAMAM-DOX caused 13.3 and $13.4 \%$ late apoptotic cells and 81.7 and $81.3 \%$ normal cells, respectively. Both HCPT loaded nanoparticles resulted in more apoptotic cells than the blank MPEG- $b$-PAMAM-DOX nanoparticles (4.7\% late apoptotic cells and $89.2 \%$ normal cells for MPEG2000- $b$-PAMAM-DOX, 4.9\% late apoptotic cells and 88.6\% normal cells for MPEG5000- $b$ PAMAM-DOX) and free HCPT (6.1\% late apoptotic cells and $88.8 \%$ normal cells) with a concentration of $1 \mu \mathrm{g} \mathrm{mL}{ }^{-1} \cdot{ }^{147}$ The advantages of co-delivery of two anticancer drugs (DOX and НСРТ), high drug loading content, $\mathrm{pH}$-dependent drug release, higher cellular uptake compared to free HCPT, and higher in vitro cytotoxicity compared to free HCPT and free DOX, make HCPT loaded MPEG- $b$-PAMAM-DOX nanoparticles attractive for drug delivery. More investigations, especially pharmacokinetic studies and in vivo antitumor efficacy, can help this system develop for cancer therapy applications. ${ }^{147}$

By comparison of the CLSM results in HeLa cells, after 3 and $16 \mathrm{~h}$ incubation with cross-linked MPEG- $b$-PAMAM-LA/ 
DOX micelles (Compound 21) (Fig. 33), it has been determined that the effective cellular uptake of the nanoparticles and efficient DOX release had taken place after a longer period of incubation. ${ }^{78}$ In fact, after cellular uptake, the acidic environment and the relatively higher GSH concentration in both endosomes and the cytoplasmic matrix triggered the cleavage of the hydrazone bonds and disulfide bonds of cross-linked MPEG- $b$-PAMAM-LA/DOX, causing the slow release of DOX. Also the weaker DOX fluorescence observed for the cells treated with cross-linked MPEG- $b$-PAMAM-LA/DOX nanoparticles in comparison with that of free DOX has been explained by slower internalization and the self-quenching effect of DOX in the nanoparticles. ${ }^{78}$

By in vitro cytotoxicity studies it has been found that crosslinked nanoparticles have been more effective after a longer period of incubation. The $\mathrm{IC}_{50}$ value of the cross-linked nanoparticles decreased from $18.9 \mu \mathrm{g} \mathrm{mL}^{-1}$ to $2.5 \mu \mathrm{g} \mathrm{mL}{ }^{-1}$ for HeLa cells, with increasing incubation time from 24 to $72 \mathrm{~h}$. The decrease of $\mathrm{IC}_{50}$ has also been shown for A549 cells from more than $20 \mu \mathrm{g} \mathrm{mL}{ }^{-1}$ to $6.3 \mu \mathrm{g} \mathrm{mL^{-1 }}$ under the same incubation conditions. Enhanced inhibition of the cell proliferation has been demonstrated for cross-linked nanoparticles incubated with GSH pretreated HeLa and A549 cells. This higher cytotoxicity has been attributed to the cleavage of disulfide crosslinking by GSH and faster release of DOX. ${ }^{78}$ Because of the high drug loading content, $\mathrm{pH}$ and reduction-sensitive DOX release behavior (regarding the acidic environment and the relatively higher GSH concentration in both endosomes and the cytoplasmic matrix), higher in vitro cytotoxic effect with the increase in incubation time, the core cross-linked MPEG- $b$ PAMAM-LA/DOX NPs showed bright prospects for anti-cancer therapy. However, investigations of in vivo efficacy, tolerable doses, and biodistribution studies are needed to complete the cross-linked MPEG- $b$-PAMAM-LA/DOX system. ${ }^{78}$

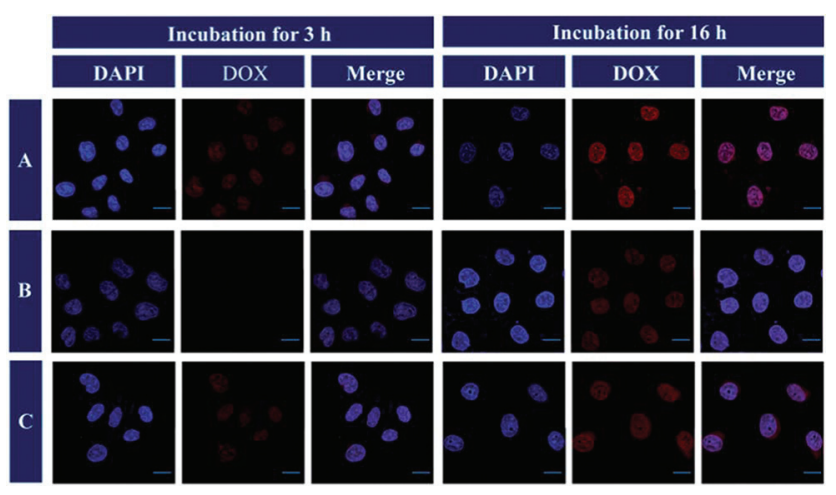

Fig. 33 Confocal laser scanning microscopy of HeLa cells $\left(1.0 \times 10^{5}\right.$ cells per well) after incubation with (A) free DOX, (B) cross-linked MPEG$b$-PAMAM-LA/DOXNPs (the cells were not pretreated) and (C) crosslinked MPEG- $b$-PAMAM-LA/DOXNPs (the cells were pretreated with $10 \mathrm{mM} \mathrm{GSH}$ ) for $3 \mathrm{~h}$ and $16 \mathrm{~h}$ at $37^{\circ} \mathrm{C}$ (DOX equivalent concentration: $0.5 \mu \mathrm{g} \mathrm{mL}{ }^{-1}$ for all formulations). The scale bars represent $20 \mu \mathrm{m}$. Reprinted with permission from ref. 78. Copyright (2014) The Royal Society of Chemistry.
5.2.3. In vivo evaluation. Biodistribution Studies of the DOX-conjugated 3-arm (PEO) star-three [G2] polyester dendritic blocks with bis-MPA units (Compound 15) performed on CD-1 female mice demonstrated no significant accumulation in any vital organ, including the liver, heart, and lungs (Fig. 34). ${ }^{136}$ This is a preferred distribution pattern compared with the free drug, which partitions into a variety of organs such as the liver and heart. Also the polymer-DOX conjugate exhibited a longer circulatory half-life (72 $\mathrm{min})$, as compared to the half-life of the free $\operatorname{drug}(8 \mathrm{~min})$, demonstrating the influence of the 3-arm PEO-polyester dendritic system in the pharmacokinetics and the distribution of the drug. ${ }^{136}$

According to the reported results, it is concluded that DOXconjugated 3-arm (PEO) star-three [G2] polyester dendritic block with bis-MPA units (Compound 16) shows advantages including biocompatibility of the carrier, pH-dependent release, which is compatible with conditions found in tumors, no significant accumulation in vital organs, and a longer circulatory half-life than the free drug. But its lower cytotoxicity in comparison with the free drug arises from hydrazone carboxylate linkages, which lead to the intramolecular cyclization reaction and subsequent poor antitumor activity. ${ }^{136,140}$

According to biodistribution studies of bow-tie polyester dendrimer-PEO ${ }^{138}$ on CD-1 female mice at a dose of approximately $40 \mathrm{mg} \mathrm{kg} \mathrm{kg}^{-1}$, no specific organ accumulation was observed for these bow-tie polymers, with a significant portion of the dose (35-46\%) found in the carcass after $48 \mathrm{~h}$. Less than $4 \%$ of the dose was found in the urine for each of these polymers, indicating that their effective sizes are above the threshold for renal filtration. After $48 \mathrm{~h}, 6-16 \%$ of the dose was excreted in the feces. Therefore, the primary route for elimination of these molecules was via intestinal excretion, believed to be the primary route by which large molecules that cannot be excreted through the kidney can escape the body. ${ }^{138}$

Biodistribution experiments of DOX-conjugated bow-tie polyester dendrimer-PEO performed in BALB/c mice bearing

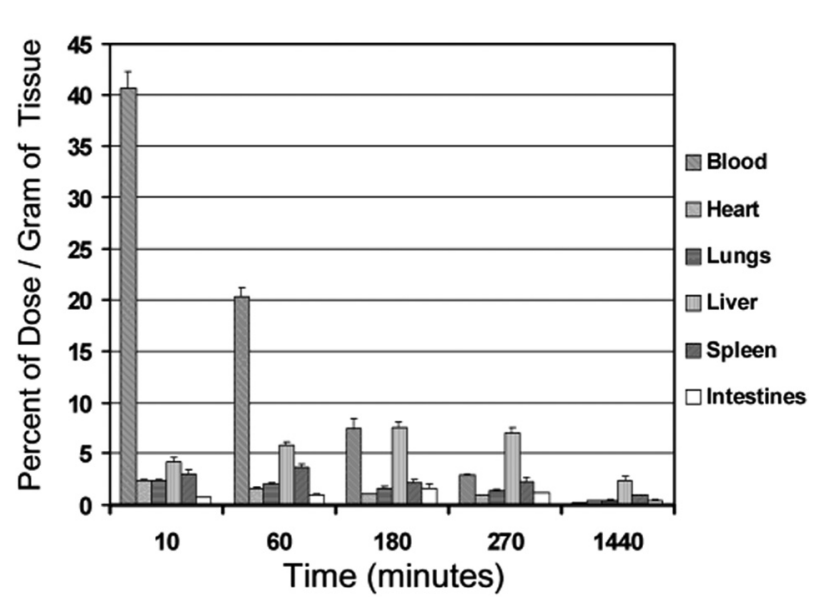

Fig. 34 Biodistribution of 3-arm PEO-polyester dendrimer/DOX conjugate. Reprinted with permission from ref. 136. Copyright (2002) American Chemical Society. 
s.c. C-26 tumors showed an elimination half-life of $16 \pm 1 \mathrm{~h}$ for the hydrazone-linked bow-tie DOX conjugate. ${ }^{139}$ In previous studies, a blood elimination half-life of $31 \pm 2 \mathrm{~h}$ was observed for a drug-free bow-tie PEO-polyester dendrimer. ${ }^{138}$ The long circulation half-life of the conjugates contrasts with the short half-life of the free drug, which is $<10 \mathrm{~min}$. The tumor concentrations of DOX measured $48 \mathrm{~h}$ after administration of either DOX-conjugated bow-tie polyester dendrimer-PEO $\left(20 \mathrm{mg} \mathrm{kg}^{-1}\right.$ DOX) or free DOX (6 mg $\left.\mathrm{kg}^{-1}\right)$ were approximately nine times higher for mice treated with DOX-conjugated bow-tie polyester dendrimer-PEO by percentage injected dose per gram of tumor. The enhanced tumor uptake of the dendrimer bound drug is a reflection of its longer circulation half-life, which exploits passive targeting by means of the EPR effect. ${ }^{139}$ Animal serum analysis determined a significant increase in the serum creatine kinase, lactic dehydrogenase, and serum transaminase values in animals that received the 40 and $60 \mathrm{mg}$ $\mathrm{kg}^{-1}$ doses of DOX-conjugated bow-tie polyester dendrimerPEO, compared with animals that received saline or the $20 \mathrm{mg}$ $\mathrm{kg}^{-1}$ dose, indicating the presence of damage to muscle tissue and to the liver at these dose levels. So it was concluded that the maximum tolerated single dose is between 20 and $40 \mathrm{mg}$ $\mathrm{kg}^{-1}$ DOX equivalents or between $\sim 200$ and $500 \mathrm{mg} \mathrm{kg} \mathrm{kg}^{-1}$ DOX-conjugated bow-tie polyester dendrimer-PEO in healthy BALB/c mice. ${ }^{139}$ To determine the optimal dosing schedule for antitumor therapy, BALB/c mice bearing s.c. C-26 tumors were administered a single dose of DOX-conjugated bow-tie polyester dendrimer-PEO (10 $\mathrm{mg} \mathrm{kg}^{-1}$ DOX) on various days after tumor inoculation. Five different groups of mice were treated with a single i.v. injection of polymer on day $2,4,8$, 12, or 16 after their tumors were implanted. The dosing schedule experiment showed that mice treated on day 8 responded the most favorably to treatment, a result that was statistically different from the mice treated on days 4,12 , and 16. A dose-response experiment was performed by monitoring the tumor growth and survival of BALB/c mice treated with a single dose of DOX-conjugated bow-tie polyester dendrimer-PEO, 8 days after implantation of a s.c. C-26 tumor. Remarkably, at the highest dose administered $\left(20 \mathrm{mg} \mathrm{kg}^{-1}\right.$ DOX equivalents), complete tumor regression was observed, resulting in $100 \%$ survival of mice in this treatment group over the 60-day experiment. In contrast, none of the free DOX $\left(6 \mathrm{mg} \mathrm{kg}^{-1}\right.$ ) administered mice were still alive on day 25 . The activity of the DOX-conjugated bow-tie polyester dendrimerPEO in vivo, despite its reduced in vitro toxicity relative to free DOX, is convincing evidence of the dendrimer's ability to modulate the pharmacokinetic profiles of attached anticancer drugs. $^{139}$

Collectively, the DOX conjugated [G-3]-( $\left.\mathrm{PEO}_{5 \mathrm{k}}\right)_{8}-[\mathrm{G}-4]-(\mathrm{OH})_{16}$ bow-tie structure showed some significant advantages, for example water solubility even at DOX concentrations of $6 \mathrm{mg}$ $\mathrm{mL}^{-1}$, pH-dependent release, no significant toxicity of the carrier, long circulation half-life of the conjugates, and enhanced tumor uptake. In spite of reduced in vitro toxicity, the DOX-conjugated bow-tie polyester dendrimer-PEO showed higher antitumor activity in vivo. These results introduce DOX- conjugated bow-tie polyester dendrimer-PEO as a promising anticancer system. ${ }^{138,139}$

The liver-targeting potential of galactose conjugated PEG- $b$ PAMAM-DOX $_{n}$ prodrug (Compound 17) has been confirmed using contrast-enhanced MRI carried out on Female ICR mice. ${ }^{141}$ Comparison of signal enhancement in liver for GalPEG- $b$-PAMAM-Gd and mPEG- $b$-PAMAM-Gd complexes showed the maximum liver ENH of both agents after $6 \mathrm{~h}$ of injection. Then, a rapid decrease of ENH has been observed for mPEG- $b$ PAMAM-Gd. In contrast, Gal-PEG- $b$-PAMAM-Gd showed a gradual decrease in ENH due to the high affinity of the ASGP receptor at the liver surface to galactosyl residues in Gal-PEG- $b$ PAMAM-Gd, proving its active liver-targeting potential. In vivo antitumor evaluations indicated inhibition of tumor growth after Gal-PEG- $b$-PAMAM-DOX ${ }_{n}$ administration (Fig. 35). GalPEG- $b$-PAMAM-DOX ${ }_{n}$ showed better in vivo antitumor efficacy than free DOX, suggesting its great potential as a polymeric antitumor prodrug. In mice treated with free DOX, an increase in tumor size was found after ten days. These data offer GalPEG- $b$-PAMAM-DOX ${ }_{n}$ as a useful targeting anticancer agent. ${ }^{141}$ Properties reported for Gal-PEG- $b$-PAMAM-DOX ${ }_{n}$, including stability in $\mathrm{pH} 7.4$, triggered drug release in $\mathrm{pH}$ 5.6-6.5 and sensitivity of the drug vehicle to lower $\mathrm{pH}$ of tumor cells, receptor mediated liver targeting, higher in vivo antitumor efficacy in spite of lower in vitro activity compared to the free drug make this system interesting for anticancer investigations. Additionally, biodistribution and pharmacokinetics studies can develop this system. ${ }^{141}$

Determined by in vivo experiments, the tumor weights in mice treated with mPEGylated L-lysine Dendron-DOX conjugate nanoparticle (Compound 18) were obviously lower compared with the tumors from the free drug DOX treatment group. ${ }^{143}$ The high antitumor activity of the DOX-conjugated nanoparticle has been attributed to the neutral charged surface, longer blood circulation, potential higher accumulation in tumors via the EPR effect and the accelerated release of DOX from endosomes. Also a smaller shift in body weight

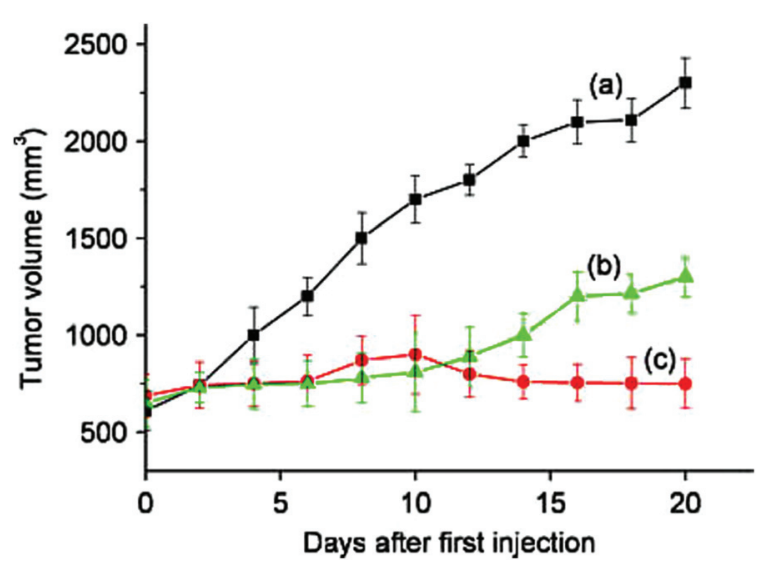

Fig. 35 In vivo antitumor efficacy of (a) PBS, (b) DOX and (c) Gal-PEG$b$-PAMAM-DOX $n$ (mean \pm SD, $n=8$ ). Reprinted with permission from ref. 141. Copyright (2010) Society of Chemical Industry. 
shift has been observed for the group administrated DOX-conjugated nanoparticles compared to the free DOX treated ones, indicating better drug tolerance. As shown in Fig. 36, histologically, for mice administrated free drug DOX, heart toxicity induced by DOX was observed due to necrosis (grade 1), acute infiltration of inflammatory cells at the epicardium and cardiac myocyte under the epicardium. In contrast, the mice administrated with drug-free peptide dendron and peptide Dendron-DOX conjugate based nanoparticles were normal and no visible difference was observed compared to the control. $^{143}$

According to in vivo experiments, higher antitumor activity has been obtained for DOX-conjugated mPEGylated L-lysine Dendron nanoparticles. ${ }^{143}$ Lower tumor weights have been observed in mice administrated with DOX-conjugated nanoparticles in comparison with the tumors from the free drug DOX treatment group. This result is caused by the neutral charged surface, longer blood circulation, potential higher accumulation in the tumor via the EPR effect and the accelerated release of DOX from endosomes. ${ }^{150}$ Also less body weight loss has been observed for the group administrated DOX-conjugated nanoparticle compared to the free DOX treated ones, indicating better drug tolerability. Histological analysis on normal mice demonstrated the heart toxicity for mice administrated free drug DOX. ${ }^{143}$ The toxicity has been attributed to

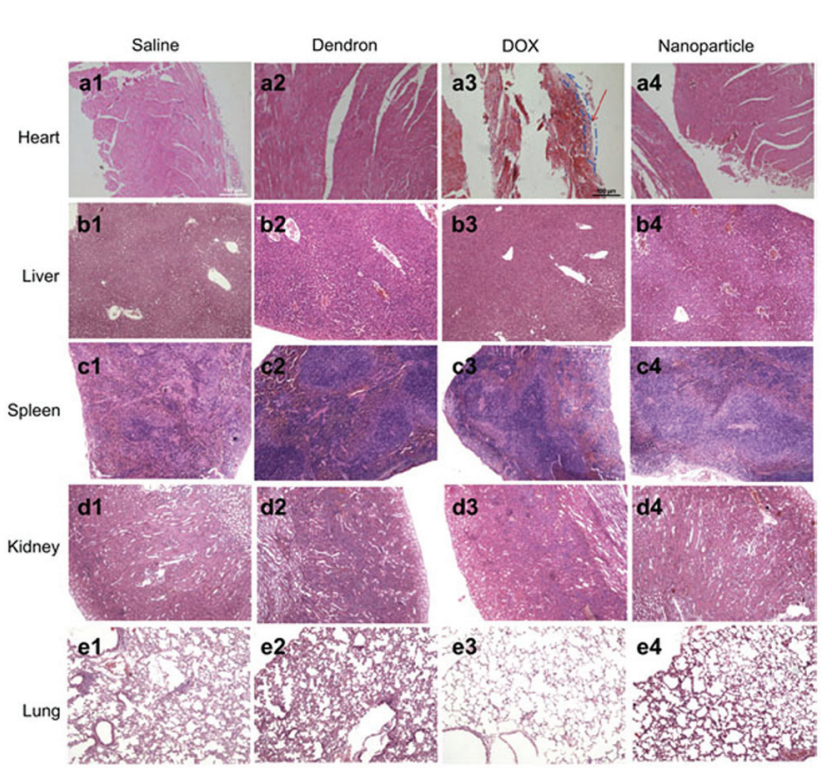

Fig. 36 Histological analysis for different organs of normal mice administrated control (Saline), drug-free peptide dendron (Dendron), free drug DOX (DOX) and mPEGylated peptide dendron-DOX conjugate based nanoparticle (Nanoparticle) (heart: $\times 200$, other tissues: $\times 100$ ). The analysis showed that the free drug DOX resulted in heart toxicity due to the observed necrosis (grade 1) with infiltration of acute inflammatory cells in the epicardium and cardiac myocyte under the epicardium (a3). In contrast, organs of mice administrated saline, drug-free mPEGylated peptide dendron and mPEGylated peptide dendron-DOX conjugate based nanoparticle did not exhibit signs of toxicity. Reprinted with permission from ref. 143. Copyright (2012) Elsevier Ltd. the necrosis (grade 1) with acute infiltration of inflammatory cells at the epicardium and cardiac myocyte under the epicardium (Fig. 36). In contrast, no toxicity has been observed in mice administrated with drug-free nanoparticles and peptide Dendron-DOX nanoparticles. The low molecular weight of the mPEG-peptide dendron and its biodegradability, high accumulation of DOX-conjugated nanoparticles in tumor tissue but lower accumulation in normal tissue via EPR effects, and the
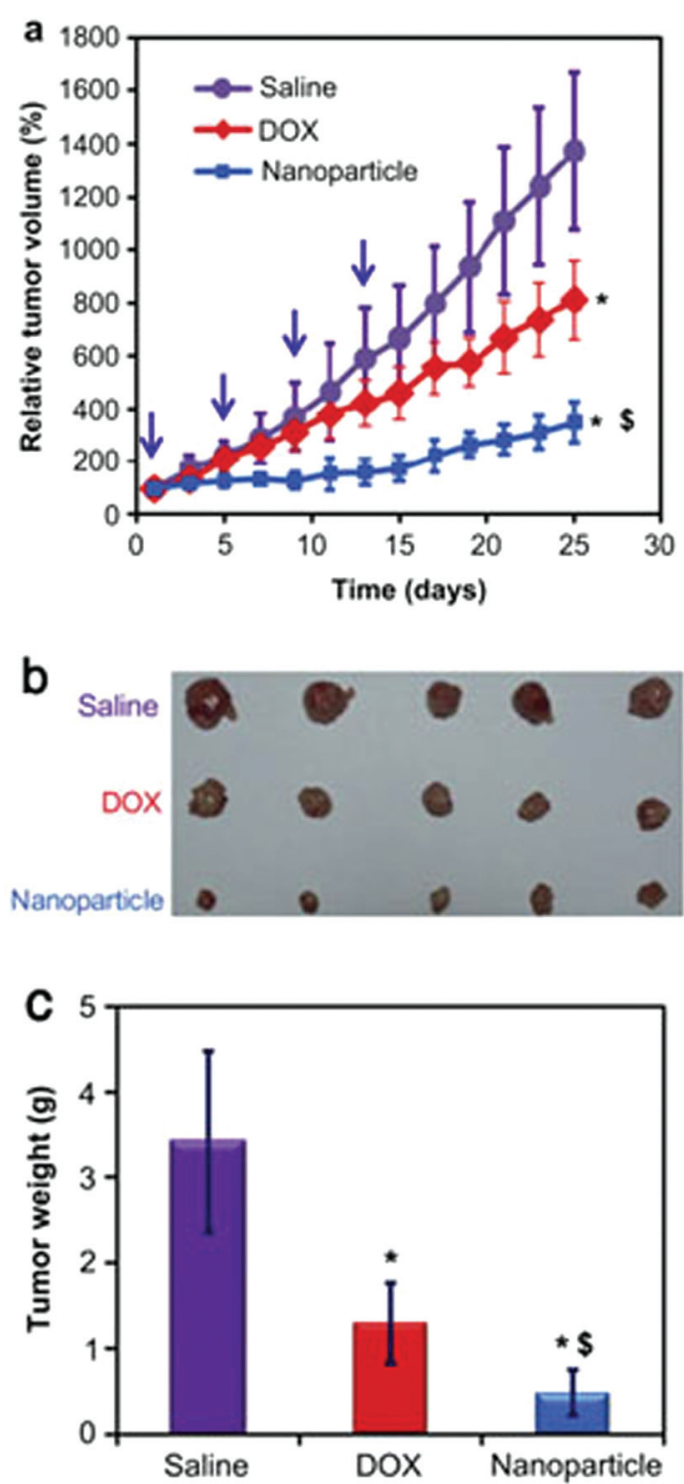

Fig. 37 In vivo tumor growth inhibition by nanoparticles. Comparison of the tumor inhibition effect of the dendronized heparin-DOX conjugate based nanoparticle with drug (Nanoparticle) versus free drug DOX (DOX) and saline in the breast tumor model $(n=5)$. The nanoparticle demonstrated significant tumor inhibition $\left({ }^{*} p<0.001\right.$, compared to Saline; ${ }^{\$} p<0.001$, compared to the free drug DOX) (a). At the end of this experiment, tumor tissues were collected from each sacrificed animal after 25 days treatment, photographed (b) and weighed $\left({ }^{*} p<0.01\right.$, compared to saline; ${ }^{\$} p<0.05$, compared to the free drug DOX) (c). Reprinted with permission from ref. 146. Copyright (2012) Elsevier Ltd. 
sensitivity of the drug vehicle to the lower $\mathrm{pH}$ of tumor cells promote clearance from the organism and thereby enhance the in vivo biocompatibility. ${ }^{143}$ The overall structural design of mPEGylated L-lysine Dendron-DOX conjugate and its properties, such as nontoxicity of the vehicle, $\mathrm{pH}$-sensitive drug release, enhanced tumor inhibition in vivo in spite of lower in vitro cytotoxicity in comparison with free DOX, better drug tolerability, enhanced in vivo biocompatibility, and reduced side effects make this prodrug a safe and efficient anticancer drug delivery system. ${ }^{143}$ From in vivo studies on mice bearing the 4T1 breast tumor model, She et al. showed that the tumors treated with dendronized heparin-DOX nanoparticle (Compound 19) exhibited a significantly stronger response than the tumors treated with saline only or free drug DOX. ${ }^{146}$ In particular, after 25 days of therapy, a statistically significant improvement was obtained for mice treated with nanoparticles, compared to the control and DOX treated groups, seen by the much smaller tumor volume, as shown in the tumor growth curves (Fig. 37a, $p<0.001$ ). The tumor sizes from mice administrated dendronized heparin-DOX conjugate based nanoparticles were obviously smaller than those from the free drug DOX treatment group and controls. (Fig. 37b), which was proportional to the observed relative tumor volume results (Fig. 37a). Simultaneously, the tumor weights in mice treated with nanoparticles were noticeably lower compared with the tumors from the free drug DOX treatment group $(p<0.05)$ and control $(p<0.001)$ (Fig. 37c). ${ }^{146}$ The high antitumor activity of the nanoparticle was attributed to the negatively charged surface, longer blood circulation, potential higher accumulation in the tumor via the EPR effect and the accelerated release of DOX from the endosomes. ${ }^{151}$ Regarding the advantages of the non-toxicity of the carrier, pH-sensitive drug release, and higher antitumor activity in vivo, dendronized heparin-DOX may therefore be a potential nanoscale drug delivery vehicle for breast cancer therapy. Certainly, pharmacokinetics studies make this system more helpful for cancer therapy purposes. ${ }^{146}$

Although these DOX-conjugated linear-dendritic polymer systems demonstrated low in vitro cytotoxicities in comparison with the free drug, ${ }^{136,141,143,146}$ the larger accumulation in tumor tissue in vivo could counter balance the low in vitro toxicity. High molecular weight polymers preferentially accumulate in solid tumor tissue due to a combination of the leaky character of tumor blood vessels formed during neo-angiogenesis

Table 4 Doxorubicin-conjugated linear-dendritic block copolymers

\begin{tabular}{|c|c|c|c|}
\hline Carrier name & Size $(\mathrm{nm})$ & In vitro activity & In vivo activity \\
\hline \multirow[t]{2}{*}{$\begin{array}{l}\text { 3-Arm PEO- } \\
\text { (bis-MPA) } \\
\text { dendrimer }\end{array}$} & $\mathrm{n}^{a}$ & $\begin{array}{l}\text { Less cytotoxicity compared to } \\
\text { the free drug on B16F10, } \\
\text { MDA-MB- } 435 \text {, and MDA-MB- } 231 \\
\text { cell line }\end{array}$ & $\begin{array}{l}\text { No significant } \\
\text { accumulation in any vital } \\
\text { organ, including the liver, } \\
\text { heart, and lungs evaluated } \\
\text { on CD- } 1 \text { female mice }\end{array}$ \\
\hline & 8 & $\begin{array}{l}\text { Lower cytotoxic activity } \\
\left(\mathrm{IC}_{50}=1.4 \pm 0.2 \mu \mathrm{g} \mathrm{m} \mathrm{m}^{-1}\right) \\
\text { compared to free DOX } \\
\left(\mathrm{IC}_{50}=0.08 \pm 0.02 \mu \mathrm{g} \mathrm{ml} \mathrm{m}^{-1}\right) \\
\text { on MDA-MB- } 231 \text { cancer cells }\end{array}$ & $\begin{array}{l}\text { Higher antitumor effects } \\
\text { compared to free DOX on } \\
\text { BALB/c mice bearing s.c. } \\
\text { C-26 tumors }\end{array}$ \\
\hline
\end{tabular}

\begin{tabular}{|c|c|c|}
\hline $\begin{array}{l}\text { Gal-PEG- } \\
b \text {-PAMAM }\end{array}$ & $\mathrm{n}^{a}$ & $\begin{array}{l}\text { Reduced cytotoxic activity } \\
\text { compared to free drug against } \\
\text { Bel-7402 cells }\end{array}$ \\
\hline $\begin{array}{l}\text { mPEGylated } \\
\text { poly(L-lysine) } \\
\text { dendron }\end{array}$ & 220 & $\begin{array}{l}\text { Lower cytotoxicity }\left(\mathrm{IC}_{50}=151 \mathrm{ng}\right. \\
\left.\mathrm{mL}^{-1}\right) \text { compared with free DOX } \\
\left(\mathrm{IC}_{50}=25.9 \mathrm{ng} \mathrm{mL}^{-1}\right) \text { on mouse } \\
\text { breast cancer cell line }(4 \mathrm{~T} 1)\end{array}$ \\
\hline $\begin{array}{l}\text { Heparin- } \\
\text { poly(L-lysine) }\end{array}$ & 90 & $\begin{array}{l}\text { Lower cytotoxicity }\left(\mathrm{IC}_{50} \text { of }\right. \\
\left.300 \mathrm{ng} \mathrm{mL}{ }^{-1}\right) \text { compared with } \\
\text { free } \mathrm{DOX}\left(\mathrm{IC}_{50} \text { of } 27 \mathrm{ng} \mathrm{mL} \mathrm{mL}^{-1}\right) \\
\text { against mouse breast cancer cell } \\
\text { line ( } 4 \mathrm{~T} 1)\end{array}$ \\
\hline $\begin{array}{l}\text { MPEG- } \\
b \text {-PAMAM }\end{array}$ & 60 & $\begin{array}{l}\text { Higher in vitro cytotoxicity for } \\
\text { nanoparticles compared to free } \\
\text { DOX and free HCPT in MCF-7 } \\
\text { and HepG } 2 \text { cell lines }\end{array}$ \\
\hline
\end{tabular}

$\begin{array}{lll}\text { MPEG- } & 140(\mathrm{CMC}= & \begin{array}{l}\text { Being more effective after a } \\ b \text {-PAMAM-LA }\end{array} \\ \left.13.30 \mu \mathrm{g} \mathrm{mL}^{-1}\right) & \begin{array}{l}\text { longer period of incubation on } \\ \text { HeLa cells and A549 cells }\end{array}\end{array}$
HeLa cells and A549 cells
Better in vivo antitumor efficacy than free DOX on female ICR mice

Higher antitumor activity in comparison with free DOX

Higher antitumor activity on mice bearing $4 \mathrm{~T} 1$ breast tumor model compare to free drug

$\mathrm{n}^{a}$

$\mathrm{n}^{a}$

\begin{tabular}{|c|c|}
\hline Advantages & Ref. \\
\hline $\begin{array}{l}\text { Biocompatibility of carrier, } \\
\text { pH-dependent release, no } \\
\text { significant accumulation in vital } \\
\text { organs, and longer circulatory } \\
\text { half-life than the free drug }\end{array}$ & 136 \\
\hline $\begin{array}{l}\text { Water solubility even at DOX } \\
\text { concentrations of } 6 \mathrm{mg} \mathrm{mL} \mathrm{mL}^{-1} \text {, } \\
\text { pH-dependent release, no } \\
\text { significant toxicity of carrier, long } \\
\text { circulation half-life of conjugates, } \\
\text { and enhanced tumor uptake and } \\
\text { higher antitumor activity in vivo }\end{array}$ & $\begin{array}{l}138 \\
139\end{array}$ \\
\hline $\begin{array}{l}\text { Stability in pH } 7.4 \text {, triggered drug } \\
\text { release in acidic } \mathrm{pH} \text {, receptor } \\
\text { mediated liver targeting, higher } \\
\text { in vivo antitumor efficacy }\end{array}$ & 141 \\
\hline $\begin{array}{l}\text { Nontoxicity of vehicle, } \mathrm{pH}^{-} \\
\text {sensitive drug release, enhanced } \\
\text { tumor inhibition in vivo, reduced } \\
\text { side effects }\end{array}$ & 143 \\
\hline Non-toxicity of carrier, pH- & 146 \\
\hline
\end{tabular}

sensitive drug release, and higher antitumor activity in vivo

Co-delivery of two anticancer drugs (DOX and HCPT), high drug loading content, pH-dependent drug release, higher cellular uptake compared to free HCPT, higher in vitro cytotoxicity High drug loading content, $\mathrm{pH}$ and reduction-sensitive DOX release behavior, higher in vitro cytotoxic effect with the elongation of the incubating time

${ }^{a}$ n: not reported. 
and to limited lymphatic drainage. The combination of these two factors is responsible for the enhanced permeability and retention effect (EPR) observed with tumor tissue, which leads to a passive targeting of drugs to tumors. In addition, the larger hydrodynamic volume of polymers contributes to the increased plasma half-life of the drug-polymer conjugates, increasing the probability of accumulation of the therapeutic agent in the tumor tissue by means of the EPR effect. Drugs have also been conjugated to polymers to improve their water solubility properties, to decrease their toxicity due to local accumulation of the drug prior to reaching the target tissue, and to protect them from possible enzymatic degradation or hydrolysis. So these DOX-conjugated linear-dendritic systems are promising because they allow slow elution of doxorubicin into the tumor after administration, since the nanoparticle has much longer blood circulation time and higher accumulation in tumor tissue due to the EPR effect (Table 4). ${ }^{136,141,143,146}$

\section{Cisplatin}

Cisplatin (cis-dichlorodiammine platinum(II)) (CDDP) is one of the most potent anticancer agents available today and is widely used in the treatment of many malignancies, including testicular, ovarian, bladder, head and neck, small cell and non-small cell lung cancers because of its potent activity in cross-linking DNA upon entering the cells. It preferentially binds to the N7 atoms of guanine bases in DNA double-helix strands, thereby preventing the strands from uncoiling and separating. This prohibits the division of the cells and ultimately results in cellular apoptosis. ${ }^{152-156}$ However, its clinical use is limited due to its significant toxic side effects, such as acute nephrotoxicity and chronic neurotoxicity. CDDP shows a rapid distribution over the whole body and high glomerular clearance within 15 min after intravenous injection. A total of $90 \%$ of the cisplatin is bound to plasma proteins in the blood and, thus, does not enter the cells; leading to lower therapeutic efficacy. Therefore, much effort has been devoted to developing a drug delivery system aimed at increasing the blood circulation period and accumulation in solid tumors. ${ }^{157-159}$

\subsection{Cisplatin-conjugated linear-dendritic block copolymers}

6.1.1. Physicochemical properties. Using linear-dendritic polycitric acid-polyethylene glycol-polycitric acid (PCA-PEGPCA) copolymers $\left(M_{\mathrm{w}} \sim 2000 \mathrm{Da}\right)$, Haririan et al. prepared conjugates of PCA-PEG-PCA-CDDP (Compound 22) in an aqueous medium. ${ }^{160}$ Drug loading of about $6 \mathrm{wt} \%$ platinum was achieved for the conjugates. By in vitro platinum release tests, it was found that the release rate in the acidic $\mathrm{pH}(5.4)$ was slightly greater and faster than the neutral $\mathrm{pH}$ (7.4), attributed to the catalytic effect of the acidic conditions on the ease of displacement of water molecules with chloride or carboxylate ions inside the cisplatin cavity. ${ }^{160}$ It was observed that when the conjugates were formed from the dendrimers, an increase in the particle size was seen in the conjugates
(141 nm), as contrasted with the dendrimers (85 nm). This phenomenon can result from the crosslinking of the dendrimers with cisplatin, which intercalates between two of the dendrimer molecules. ${ }^{160}$

In our previous study, we used polycitric acid-polyethylene glycol-polycitric acid (PCA-PEG-PCA) linear-dendritic copolymers to solubilize and functionalize multi-walled carbon nanotubes (MWCNTs) by noncovalent interactions. ${ }^{161}$ We showed that a potential anticancer drug cisplatin can be conjugated to the carboxyl functional groups of the dendritic blocks of PCAPEG-PCA linear-dendritic copolymers and then the prodrugs interacted with the MWCNTs noncovalently, leading to formation of MWCNT/PCA-PEG-PCA-CDDP hybrid nanomaterial-based drug delivery systems (HNDDSs) (Compound 23). Based on our previous investigations, the cavity of the polymer-functionalized carbon nanotubes is able to host nanoparticles of up to $15 \mathrm{~nm}$ in diameter, due to their high solubility and opened cavity. Since the size of an individual PCAPEG-PCA linear-dendritic copolymer is less than $10 \mathrm{~nm},{ }^{162,163}$ they can transfer conjugated CDDP molecules to the cavity of MWCNT/PCA-PEG-PCA hybrid nanomaterials (Fig. 37b). As shown in the TEM images, the synthesized hybrid nanomaterials "transfer" CDDP molecules, not only by conjugation to the linear-dendritic copolymers on their surface but also in their cavity. Drug release studies at $37{ }^{\circ} \mathrm{C}$ and $\mathrm{pH} 7.4$ showed a slow release rate, with cumulative release percentage around $40 \%$ after $168 \mathrm{~h}$ (Fig. 38). ${ }^{161}$

In another study, we reported the conjugation of CDDP with PCA-PEG-PCA/CNT $/ \gamma-\mathrm{Fe}_{2} \mathrm{O}_{3} \mathrm{NP}$ hybrid nanomaterials (Compound 23). ${ }^{164}$ Deposition of $\gamma-\mathrm{Fe}_{2} \mathrm{O}_{3}$ nanoparticles onto the surface of CNTs led to magnetic CNT $/ \gamma-\mathrm{Fe}_{2} \mathrm{O}_{3} \mathrm{NP}$, and then non-covalent interactions between PCA-PEG-PCA and CNT/ $\gamma-\mathrm{Fe}_{2} \mathrm{O}_{3} \mathrm{NP}$ resulted in PCA-PEG-PCA/CNT $/ \gamma-\mathrm{Fe}_{2} \mathrm{O}_{3} \mathrm{NP}$ hybrid nanomaterials with improved water solubility, functionality, and potential application to target anticancer drugs. According to TGA analysis, the weight percent of PCA-PEG-PCA lineardendritic copolymers absorbed onto the surface of CNTs is around $45 \%$. While the molecular weight of CNTs is much more than that of linear-dendritic copolymers, it can be found that a large number of linear-dendritic copolymers are attached onto the surface of a CNT. ${ }^{164}$ Based on TGA thermal
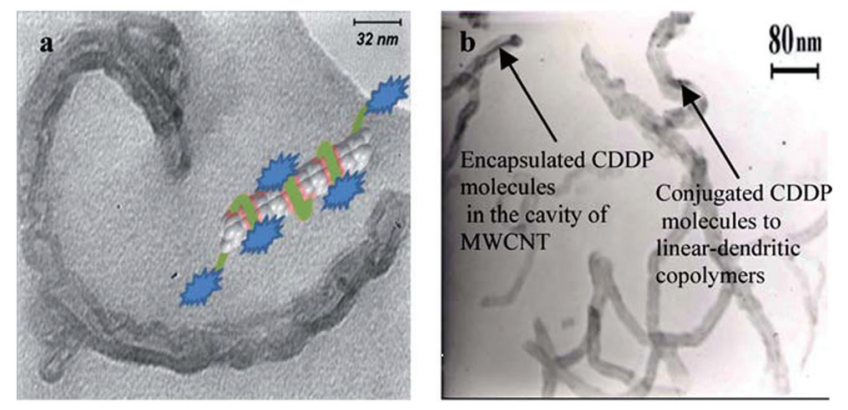

Fig. 38 TEM images of (a) MWCNT/PCA-PEG-PCA and (b) MWCNT/ PCA-PEG-PCA-CDDP. Reprinted with permission from ref. 161. Copyright (2011) The Royal Society of Chemistry. 
analysis, the weight percent of CDDP in the CDDP/PCA-PEG$\mathrm{PCA} / \mathrm{CNT} / \gamma-\mathrm{Fe}_{2} \mathrm{O}_{3} \mathrm{NP}$ drug delivery system is around $7 \%$. DLS experiments show that the average diameter of $\mathrm{CNT} / \gamma-\mathrm{Fe}_{2} \mathrm{O}_{3} \mathrm{NP}$ hybrid nanomaterials in water changes from 977 to $190 \mathrm{~nm}$ upon interaction with PCA-PEG-PCA linear-dendritic copolymers, confirming that the conformation of CNTs converts from the linear to the globular form. This is of great importance, because the shape and size of nanomaterial- and especially CNT-based drug delivery systems affect their toxicity significantly, such that the carcinogenicity of CNTs is sometimes ascribed to their length, and has been compared to asbestos fibers. It should be kept in mind that another reason for the decrease in size of $\mathrm{CNT} / \gamma-\mathrm{Fe}_{2} \mathrm{O}_{3} \mathrm{NP}$ hybrid nanomaterials could be the separation of their bundles toward individual objects, due to the noncovalent interactions with linear-dendritic copolymers. The CDDP/PCA-PEG-PCA/CNT $/ \gamma-\mathrm{Fe}_{2} \mathrm{O}_{3} \mathrm{NP}$ drug delivery system in water is $11 \mathrm{~nm}$ bigger than the PCAPEG-PCA $/ \mathrm{CNT} / \gamma-\mathrm{Fe}_{2} \mathrm{O}_{3} \mathrm{NP}$ hybrid nanomaterial, proving that the interaction of the CDDP/PCA-PEG-PCA anticancer prodrug with the surface of $\mathrm{CNT} / \gamma-\mathrm{Fe}_{2} \mathrm{O}_{3} \mathrm{NP}$ is weaker than that of the PCA-PEG-PCA linear-dendritic copolymer. This result confirms that CDDP molecules are conjugated with the carboxyl functional groups of PCA blocks and limit interactions between linear-dendritic copolymers and hydroxyl functional groups of iron oxide nanoparticles anchored onto the surface of CNTs. ${ }^{164}$ VSM curves showed that the saturation of magnetization of PCA-PEG-PCA/CNT/ $\gamma-\mathrm{Fe}_{2} \mathrm{O}_{3} \mathrm{NP}$ hybrid nanomaterials was smaller than that of $\mathrm{CNT} / \gamma-\mathrm{Fe}_{2} \mathrm{O}_{3} \mathrm{NP}$, but both had similar properties (Fig. 39). This proved that the magnetic properties of $\mathrm{CNT} / \gamma-\mathrm{Fe}_{2} \mathrm{O}_{3} \mathrm{NP}$ were not influenced by the self-assembly of PCA-PEG-PCA linear-dendritic copolymers on their surfaces, indicating that the PCA-PEG-PCA/CNT $/ \gamma-\mathrm{Fe}_{2} \mathrm{O}_{3} \mathrm{NP}$ hybrid nanomaterial can be used as a promising material in cancer diagnosis and therapy. ${ }^{164}$

6.1.2. In vitro evaluations. In vitro cytotoxicity assay evaluated in CT26 cells (24 h incubation) demonstrated an $\mathrm{IC}_{50}$ of $0.8252 \mu \mathrm{g} \mathrm{mL}^{-1}$ for PCA-PEG-PCA (G2)-CDDP conjugates

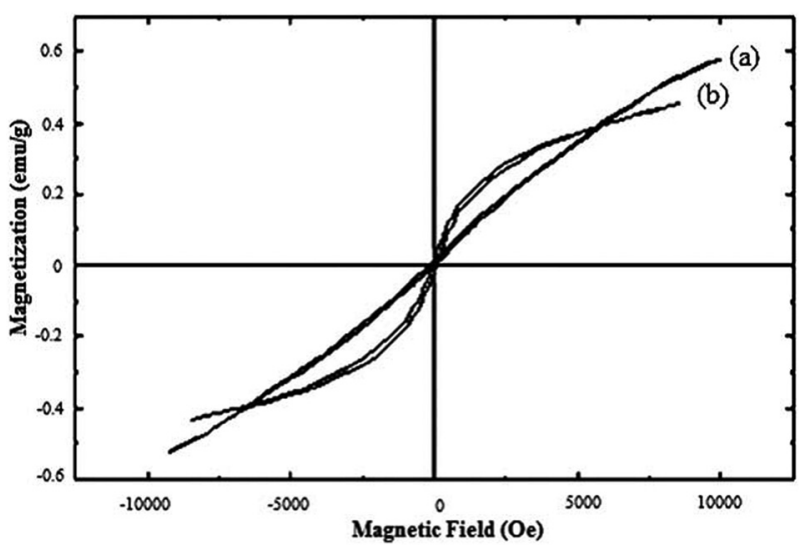

Fig. 39 Hysteresis loop by VSM of: (a) $\mathrm{CNT} / \gamma-\mathrm{Fe}_{2} \mathrm{O}_{3} \mathrm{NP}$ and (b) PCA$\mathrm{PEG}-\mathrm{PCA} / \mathrm{CNT} / \gamma-\mathrm{Fe}_{2} \mathrm{O}_{3} \mathrm{NP}$. Reprinted with permission from ref. 164. Copyright (2011) The Royal Society of Chemistry.
(Compound 22), which was 9 fold lower than that of free cisplatin. IC $_{50}$ determined for PCA-PEG-PCA(G2)-CDDP conjugates in HT1080 cell lines (48 h incubation) was $0.973 \mu \mathrm{g}$ $\mathrm{mL}^{-1}$, which was 8.4 fold lower than that determined for free cisplatin. ${ }^{160}$ These significantly higher toxicities were explained by two factors: (1) higher uptake of the conjugates as contrasted with the free cisplatin, due to the citric acid content of the conjugates, together with the greater demands of cancer cells for such energy sources during the time of incubation. (2) Increased liberation of the drug, attributed to lysosomal enzymes existing inside the cell, which gradually break down the bond between cisplatin and the dendrimers. ${ }^{160}$

Regarding the nontoxicity of PCA-PEG-PCA and the in vitro results gained for the conjugates of cisplatin-PCA-PEG-PCA, including greater and faster drug release rate in acidic $\mathrm{pH}$, and greater cytotoxicity compared to free cisplatin, it is hoped that these conjugates would be able to maintain the observed potency in vivo and retain the parent drug conjugated at the surface of the dendrimers in physiological plasma conditions. Future in vivo studies will be able to clarify the potentiality of these entities in the cure of both sensitive and resistant cancerous cells. $^{160}$

In vitro cytotoxicity studies on murine colon adenocarcinoma tumor C26 cancer cells demonstrated higher cytoxicity for MWCNT/PCA-PEG-PCA-CDDP HNDDSs (Compound 23) in comparison with the free drug. ${ }^{161}$ This was attributed to the complementary roles of carbon nanotubes and PCA-PEG-PCA linear-dendritic copolymers. MWCNTs raise the rate of "transferring" of the linear-dendritic copolymers from the cell membrane. On the other hand, PCA-PEGPCA linear-dendritic copolymers improve the water solubility of the MWCNTs and, due to their citric acid backbone PCAPEG-PCA, can probably be used as the source of energy by the cells that cause to insert MWCNTs in the cell metabolism. ${ }^{161}$ In summary, the results from this study showed a slow rate of cisplatin release at physiological conditions and a higher cytotoxicity for MWCNT/PCA-PEG-PCA-CDDP in comparison with the free drug, which make this system useful for anticancer drug delivery. More studies in pharmacokinetics and the antitumor effect in vivo are required for developing this system in cancer therapy. ${ }^{161}$

In vitro cytotoxicity tests conducted on the mouse tissue connective fibroblast adhesive cell line (L929) demonstrated that a $100 \mu \mathrm{g} \mathrm{ml} \mathrm{m}^{-1}$ concentration of CDDP/PCA-PEG-PCA/ $\mathrm{CNT} / \gamma-\mathrm{Fe}_{2} \mathrm{O}_{3} \mathrm{NP}$ (Compound 24) killed more than 95\% of cancer cells (Fig. 40). ${ }^{164}$ This high toxicity was explained by fast transfer through the cell membrane caused by CNT, and high water solubility and ability to insert into the cell metabolism caused by PCA-PEG-PCA. ${ }^{164}$

Noncovalent interactions between carbon nanotubes and linear-dendritic copolymers lead to hybrid nanomaterials having a hybrid of properties such as fast transfer through the cell membrane, high functionality, water solubility, biocompatibility, and ability to target drugs to tumors (Fig. 41). Sufficient in vitro cytotoxicity makes this system attractive for future in vivo studies (Table 5). ${ }^{164}$ 


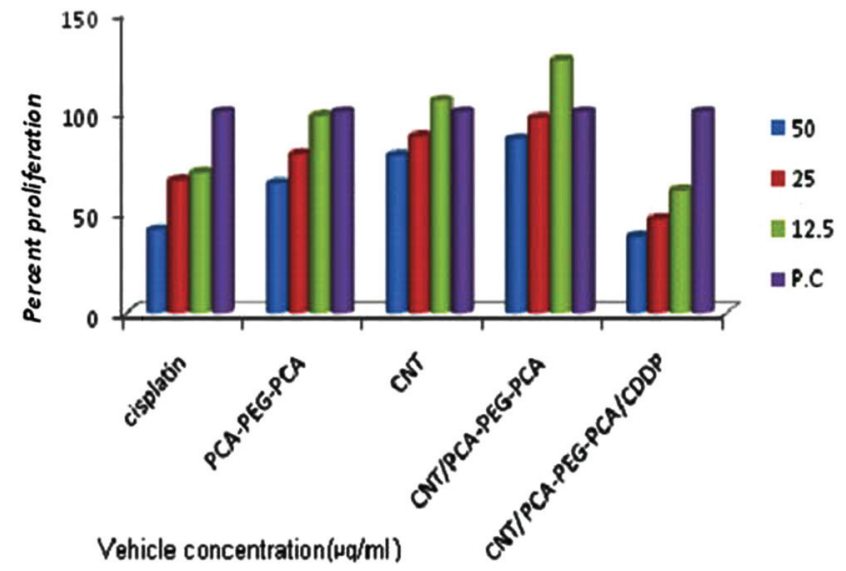

Fig. 40 Percentage survival of C26 cancer cells, assessed by the MTT assay, after exposure to free CDDP, opened MWCNT, PCA-PEG-PCA, MWCNT/PCA-PEG-PCA and MWCNT/PCA-PEG-PCA-CDDP at 12.5, 25 and $50 \mu \mathrm{g} \mathrm{mL}^{-1}(n=3)$. P.C. is the positive control. Reprinted with permission from ref. 161. Copyright (2011) The Royal Society of Chemistry.

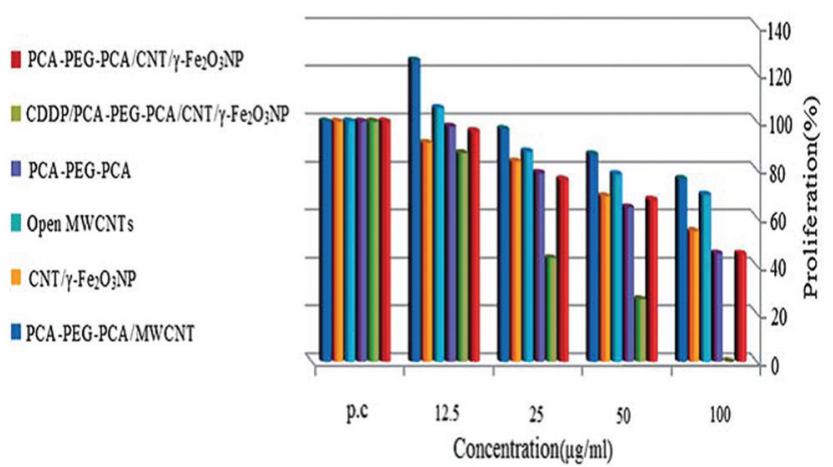

Fig. 41 The MTT assay results for opened MWCNT, CNT $/ \gamma-\mathrm{Fe}_{2} \mathrm{O}_{3} \mathrm{NP}$, PCA-PEG-PCA, PCA-PEG-PCA/CNT, PCA-PEG-PCA/CNT/ $\gamma-\mathrm{Fe}_{2} \mathrm{O}_{3} \mathrm{NP}$ and CDDP/PCA-PEG-PCA/CNT/ $\gamma-\mathrm{Fe}_{2} \mathrm{O}_{3} \mathrm{NP}$ hybrid nanomaterials. Reprinted with permission from ref. 164. Copyright (2011) The Royal Society of chemistry.

\section{Camptothecin}

Camptothecin (CPT), a natural plant alkaloid extracted from Camptotheca acuminate, is a promising antitumor agent that acts by stabilizing a topoisomerase I-induced single strand break in the phosphodiester backbone of DNA, thereby preventing religation. This causes the destruction of DNA strands during DNA replication in the cell cycle, which leads to cell death if the broken DNA is not repaired. ${ }^{165-167}$ The drug is a pentacyclic indole alkaloid, with the terminal ring converting readily between the lactone in acidic environments $(\mathrm{pH}<5)$ to the carboxylate $(\mathrm{pH}>8)$ form. In order for CPT to be active, the lactone form must dominate. The opening of the lactone ring at physiological $\mathrm{pH}$ and above, which produces the less active and highly toxic carboxylate form, prevents the clinical application of CPT in cancer therapy. Moreover, poor solubility in water and in physiologically acceptable organic solvents restricts practical use of the active lactone form of CPT. ${ }^{168-170}$ Attempts to overcome these limitations have involved the conjugation of camptothecin to biocompatible polymers (prodrug approach), ${ }^{171}$ and encapsulation into liposomes, ${ }^{172}$ polymeric micelles, ${ }^{173}$ dendrimers, ${ }^{174}$ and nanoparticles. ${ }^{175}$

\subsection{Camptothecin-conjugated linear-dendritic block copolymers}

7.1.1. Physicochemical properties. Camptothecin has been conjugated to PEG-block-dendritic polylysine to tailor the hydrophobicity of amphiphilic linear-dendritic PEG-polylysine-CPT conjugates (Compound 25) ${ }^{79}$ By CPT contentcontrolled self-assembly, nanostructures - nanospheres or nanorods of different diameters and lengths, have been obtained. As shown in Fig. 42, CPT-PDP containing a disulfide bond and an NHS active ester group has been reacted with PEG-block-dendritic polylysine $\left(\mathrm{PEG}_{45}-\mathrm{DPLL}-\mathrm{G}_{2}\right)$ of different generations. The CPT contents of PEG-polylysine $\mathrm{G}_{0}-\mathrm{CPT}$, PEG-polylysine $\mathrm{G}_{1}$-DiCPT, PEG-polylysine $\mathrm{G}_{2}$-TetraCPT and PEG-polylysine $\mathrm{G}_{3}$-OctaCPT were $13.4 \%, 21.4 \%, 30.6 \%$, and $38.9 \%$ by weight, respectively.

Table 5 Cisplatin-conjugated linear-dendritic block copolymers

\begin{tabular}{|c|c|c|c|c|c|}
\hline Carrier name & $\begin{array}{l}\text { Size } \\
(\mathrm{nm})\end{array}$ & In vitro activity & $\begin{array}{l}\text { In vivo } \\
\text { activity }\end{array}$ & Advantages & Ref. \\
\hline $\begin{array}{l}\text { PCA-PEG- } \\
\text { PCA }\end{array}$ & 141 & $\begin{array}{l}\text { Significantly higher cytotoxicity in CT26 cells }\left(\mathrm{IC}_{50}\right. \\
\text { of } 0.8252 \mu \mathrm{g} \mathrm{mL} \mathrm{m}^{-1} \text { for conjugates, } 9 \text { fold lower than } \\
\text { free cisplatin) and in HT1080 cell lines (IC } \mathrm{IC}_{50} \text { of } \\
0.973 \mu \mathrm{g} \mathrm{mL} \mathrm{m}^{-1} \text { for conjugates, } 8.4 \text { fold lower than } \\
\text { free cisplatin) }\end{array}$ & $\mathrm{n}^{a}$ & $\begin{array}{l}\text { Nontoxicity of carrier, greater and faster drug } \\
\text { release rate in acidic pH, and greater } \\
\text { cytotoxicity compared to free cisplatin }\end{array}$ & 160 \\
\hline $\begin{array}{l}\text { MWCNT/ } \\
\text { PCA-PEG- } \\
\text { PCA }\end{array}$ & 371 & $\begin{array}{l}\text { Higher cytotoxicity for HNDDSs in comparison with } \\
\text { the free drug on murine colon adenocarcinoma } \\
\text { tumor C } 26 \text { cancer cells }\end{array}$ & $\mathrm{n}^{a}$ & $\begin{array}{l}\text { Slow rate of cisplatin release at physiological } \\
\text { conditions and higher cytotoxicity compared } \\
\text { to the free drug }\end{array}$ & 161 \\
\hline $\begin{array}{l}\text { PCA-PEG- } \\
\text { PCA/CNT/ } \\
\gamma-\mathrm{Fe}_{2} \mathrm{O}_{3}\end{array}$ & 200 & $\begin{array}{l}\text { Higher cytotoxicity against the mouse tissue } \\
\text { connective fibroblast adhesive cell line (L929) }\end{array}$ & $\mathrm{n}^{a}$ & $\begin{array}{l}\text { Fast transfer through the cell membrane, high } \\
\text { functionality, water solubility, } \\
\text { biocompatibility, sufficient in vitro cytotoxicity }\end{array}$ & 164 \\
\hline
\end{tabular}




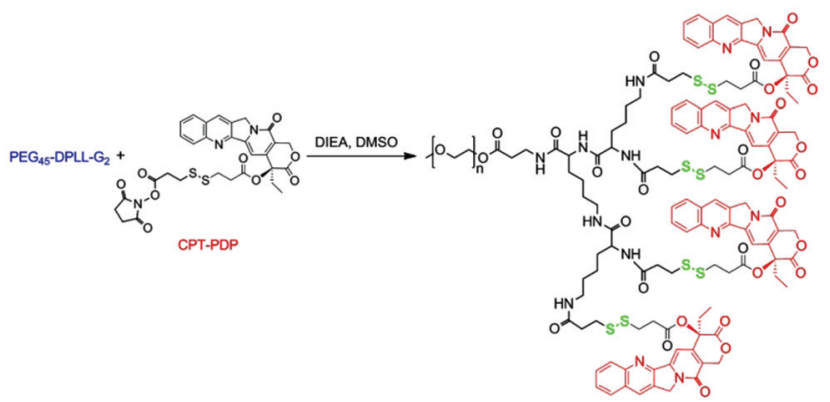

Fig. 42 Schematic illustration of PEG-dendritic polylysine (G2)-camptothecin conjugation reaction (Compound 25). Reprinted with permission from ref. 79. Copyright (2013) Elsevier Ltd.

PEG-polylysine $\mathrm{G}_{0}-\mathrm{CPT}$ and PEG-polylysine $\mathrm{G}_{1}$-DiCPT formed uniform $100 \mathrm{~nm}$ nanospheres. Interestingly, PEG45TetraCPT and PEG45-OctaCPT formed unusual nanorods. The nanorods of PEG-polylysine $\mathrm{G}_{2}$-TetraCPT were about $60 \mathrm{~nm}$ in diameter and $500 \mathrm{~nm}$ long, and those of PEG-polylysine $\mathrm{G}_{3}{ }^{-}$ OctaCPT were about $100 \mathrm{~nm}$ in diameter and about $1 \mathrm{~mm}$ long. The stability of the nanostructures was studied in PBS at $37{ }^{\circ} \mathrm{C}$ by DLS. PEG45-DiCPT nanospheres and PEG45-TetraCPT nanorods were stable for over five days and their sizes did not change over time, whereas PEG45-OctaCPT nanorods aggregated slightly. None of these nanostructures released any CPT under these conditions. In vitro drug release studies showed no CPT release from PEG-polylysine-CPT formulations due to the conjugation of CPT molecules by disulfide bonds, which can be cleaved intracellularly by GSH, indicating stability of formulations at physiological conditions but quickly releasing the drug CPT once in the cytosol. GSH-mediated release was evidenced by the addition of DTT, a strong reducing agent similar to GSH, which caused the immediate release of CPT-thioester (CPT-SH). ${ }^{79}$

7.1.2. In vitro evaluations. MTT assay on MCF-7 cells determined the $\mathrm{IC}_{50}$ value $0.138 \mu \mathrm{g} \mathrm{mL}{ }^{-1}$ for PEG45-polylysine $\mathrm{G}_{1}$-DiCPT, $0.073 \mu \mathrm{g} \mathrm{mL} \mathrm{mL}^{-1}$ for PEG45-polylysine $\mathrm{G}_{2}$-TetraCPT and $0.070 \mu \mathrm{g} \mathrm{mL}^{-1}$ for PEG45-polylysine $\mathrm{G}_{3}$-OctaCPT, which are higher than that of free CPT $\left(0.008 \mu \mathrm{g} \mathrm{mL}{ }^{-1}\right) .{ }^{79}$

7.1.3. In vivo evaluations. Pharmacokinetics in $\mathrm{BALB} / \mathrm{c}$ mice determined elimination half-life times $\left(t_{1 / 2}\right)$ of $5.82 \mathrm{~h}$ for PEG-polylysine $\mathrm{G}_{2}$-TetraCPT, which was significantly greater than those of PEG-polylysine $\mathrm{G}_{1}$-DiCPT $(1.61 \mathrm{~h})$ and PEGpolylysine $\mathrm{G}_{3}$-OctaCPT $(1.70 \mathrm{~h}){ }^{79}$ The prolonged circulation time of the PEG-polylysine $\mathrm{G}_{2}$-TetraCPT nanorods has been attributed to their elongated shape, which might align or tumble in the flow to reduce clearance by the liver or spleen. Biodistribution studies after $4 \mathrm{~h}$ i.v. administration to BALB/c mice demonstrated the presence of nanostructures in the spleen. Also accumulation of some PEG-polylysine $\mathrm{G}_{1}$-DiCPT in the liver $(15.75 \pm 3.85 \%$ ID per $\mathrm{g}$ tissue $)$, and a significant amount of PEG-polylysine $\mathrm{G}_{3}$-OctaCPT in the lung $(64.89 \pm$ $2.63 \%$ ) was observed. ${ }^{79}$ PEG-polylysine $\mathrm{G}_{2}$-TetraCPT had lower concentrations in the liver and spleen than PEG-polylysine
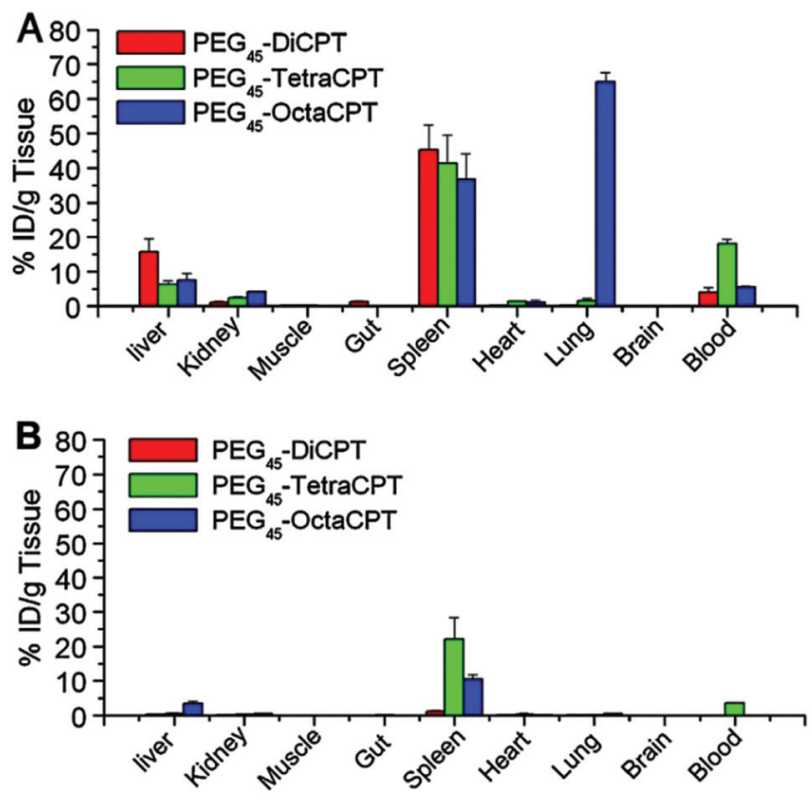

Fig. 43 The biodistribution at $4 \mathrm{~h}(\mathrm{~A})$ or $24 \mathrm{~h}$ (B) post i.v. administration of the PEG-xCPT nanostructures. Dose, $10 \mathrm{mg} \mathrm{CPT-eq.} \mathrm{kg}^{-1}, n=4$. Reprinted with permission from ref. 79. Copyright (2013) Elsevier Ltd.

$\mathrm{G}_{1}$-DiCPT. After $24 \mathrm{~h}$, PEG-polylysine $\mathrm{G}_{1}$-DiCPT almost disappeared from all the organs. The level of PEG-polylysine $\mathrm{G}_{3}$-OctaCPT in the lung was also greatly reduced and little remained in the spleen $(10.53 \pm 1.31 \%$ ID per $g$ of tissue $)$ and liver $\left(3.55 \pm 0.57 \%\right.$ ID per $g$ tissue). PEG-polylysine $\mathrm{G}_{2}-$ TetraCPT was still found in the spleen $(22.05 \pm 6.33 \%$ ID per $g$ tissue) and blood (3.59 $\pm 0.29 \%$ ID per $g$ blood). ${ }^{79}$ Collectively, high drug content, stability of formulations under physiological conditions, and a reduction-sensitive drug release profile, which leads to fast release in cytosol, made this system suitable for drug delivery. It was demonstrated that PEG-polylysine $\mathrm{G}_{2}$-TetraCPT conjugate nanorods with proper lengths can unite the two opposites in cancer-drug delivery: long blood circulation versus fast cellular uptake and drug retention in circulation versus intracellular drug release, ideal for efficient tumor-drug delivery (Fig. 43). ${ }^{79}$

\section{Conclusion}

In summary, linear-dendritic block copolymers show great potential in anticancer drug delivery applications. The high architectural control and the option to tailor the properties of the linear-dendritic copolymers to the specific requirements of cancer therapy, including prolonged circulation times, increased drug solubility, reduced drug toxicity, selective delivery to tumors by active targeting with covalently bonded tumor-targeting agents, or passive targeting, resulting from the enhanced permeability and retention (EPR) effect, make these nanostructures promising carriers for a variety of anticancer drugs. The reported data demonstrate that in most 
Table 6 Camptothecin-conjugated linear-dendritic block copolymers

\begin{tabular}{|c|c|c|c|c|c|c|}
\hline $\begin{array}{l}\text { Carrier } \\
\text { name }\end{array}$ & $\begin{array}{l}\text { Size } \\
(\mathrm{nm})\end{array}$ & CMC & In vitro activity & In vivo activity & Advantages & Ref. \\
\hline $\begin{array}{l}\text { PEG- } \\
\text { polylysine }\end{array}$ & 100 & $\begin{array}{l}\text { PEG45-polylysine G1-DiCPT } \\
=0.114 \mathrm{mg} \mathrm{mL}^{-1}, \text { PEG45- } \\
\text { polylysine G2-TetraCPT = } \\
0.074 \mathrm{mg} \mathrm{mL}^{-1}, \text { PEG45- } \\
\text { polylysine G3-OctaCPT }= \\
0.025 \mathrm{mg} \mathrm{mL}^{-1}\end{array}$ & 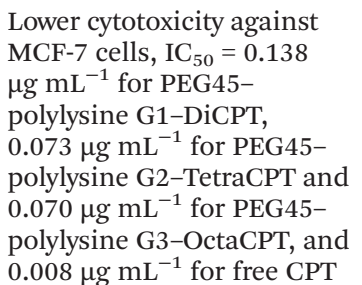 & $\begin{array}{l}\text { After } 24 \mathrm{~h} \text { i.v. } \\
\text { administration to BALB/c } \\
\text { mice, greatly reduced level } \\
\text { of PEG-polylysine } \\
\mathrm{G}_{3} \text {-OctaCPT in the lung, } \\
\text { spleen, and liver. But the } \\
\text { remaining PEG-polylysine } \\
\mathrm{G}_{2} \text {-TetraCPT was in the } \\
\text { spleen and blood }\end{array}$ & $\begin{array}{l}\text { High drug content, } \\
\text { stability of formulations } \\
\text { under physiological } \\
\text { conditions, and } \\
\text { reduction-sensitive drug } \\
\text { release }\end{array}$ & 79 \\
\hline
\end{tabular}

studies PEG has been utilized as a linear segment of lineardendritic anticancer carriers, because of its biocompatibility and hydrophilic properties. The chemotherapeutic agents can be loaded either as conjugated to the functional groups on the dendritic blocks or encapsulated by the hydrophobic interior of the dendritic segments of block copolymers. Several strategies have been employed for the delivery of loaded chemotherapeutic agents by different modes, such as $\mathrm{pH}-$, enzyme-, light-, and glutathione-dependent. In many cases, slow drug release in physiological conditions, improved selectivity and higher drug accumulation in tumor tissues, and reduced drug toxicities were observed for drug loaded lineardendritic copolymers, indicating these nanostructures as promising vehicles for anticancer agents. Although a large number of studies, investigating the in vitro and in vivo antitumor efficacy of anticancer drugs loaded by linear-dendritic copolymers are available, more research focusing on the biodistribution, pharmacokinetic studies, toxicity problems and side effects is needed. Clearly, more studies lead to better knowledge in the design of linear-dendritic based anticancer drug delivery systems, in order to achieve better targeting properties and higher antitumor effects, and to avoid the uptake in vital organs and side effects that can be used to assist in the optimization of clinical protocols (Table 6).

$\begin{array}{ll}\text { ALN } & \text { Alendronate } \\ \text { bis-MPA } & \text { 2,2-Bis(hydroxymethyl) propanoic acid } \\ \text { MWCNTs } & \text { Multi-walled carbon nanotubes } \\ \text { PG } & \text { Polyglycerol } \\ \text { Bz } & \text { Benzylidene } \\ \text { DNQ } & \text { Diazonaphthoquinone } \\ \text { NIR } & \text { Near-infrared light-responsive } \\ \text { PDMAEMA } & \text { Poly(2-dimethylaminoethyl methacrylate) } \\ \text { PNIPAM } & \text { Poly(N-isopropylacrylamide) } \\ \text { PDMAEMA } & \text { Poly(2-(dimethylamino)ethyl methacrylate) } \\ \text { PPEGMA } & \text { Poly(poly(ethylene glycol) methyl ether methacrylate) } \\ \text { PCL } & \text { Poly(z-caprolactone) } \\ \text { LA } & \text { Lipoic acid } \\ \text { PCA } & \text { Polycitric acid } \\ \text { ENH } & \text { Enhanced value } \\ \text { ASGP } & \text { Asialo-glycoprotein receptor } \\ \text { FR } & \text { Folate receptor } \\ \text { GSH } & \text { Glutathione } \\ \text { LA } & \text { Lipoic acid } \\ \text { HNDDSs } & \text { Hybrid nanomaterial-based drug delivery systems } \\ \text { PDI } & \text { Polydispersity index } \\ \text { NIRF } & \text { Near infrared fluorescence imaging } \\ \text { EPR } & \text { Enhanced permission and retention effect } \\ \text { MTD } & \text { Most tolerated dosage } \\ \text { RES } & \text { Reticuloendothelial system }\end{array}$

\section{Acknowledgements}

Authors would like to thank Iran Nano Council to support this work financially.

\section{Notes and references}

1 Y. Y. Diao, H. Y. Li, Y. H. Fu, M. Han, Y. L. Hu, H. L. Jiang, Y. Tsutsumi, Q. C. Wei, D. W. Chen and J. Q. Gao, Int. J. Nanomed., 2011, 6, 1955.

2 C. Oerlemans, W. Bult, M. Bos, G. Storm, J. F. Nijsen and W. E. Hennink, Pharm. Res., 2010, 27, 2569.

3 M. López-Gómez, E. Malmierca, M. de Górgolas and E. Casado, Crit. Rev. Oncol./Hematol., 2013, 88, 117. 
4 L. Brannon-Peppas and O. J. Blanchette, Adv. Drug Delivery Rev., 2004, 56, 1649.

5 S. S. Feng and S. Chien, Chem. Eng. Sci., 2003, 58, 4087.

6 X. Zeng, R. Morgenstern and A. M. Nyström, Biomaterials, 2014, 35, 1227.

7 L. Nobs, F. Buchegger, R. Gurny and E. Allémann, Bioconjugate Chem., 2006, 17, 139.

8 C. Clementi, K. Miller, A. Mero, R. Satchi-Fainaro and G. Pasut, Mol. Pharm., 2011, 8, 1063.

9 D. Brambilla, P. Lucianni and J.-C. Leroux, J. Controlled Release, 2014, 190, 9.

10 M. Adeli, R. S. Sarabi, R. Y. Farsi, M. Mahmoudi and M. Kalantari, J. Mater. Chem., 2011, 21, 18686.

11 S. Lu, K. G. Neoh, C. Huang, Z. Shi and E. T. Kang, J. Colloid Interface Sci., 2013, 412, 46.

12 A. Krais, L. Wortmann, L. Hermanns, M. Feliu, M. Vahter, S. Stucky, M. Mathur and B. Fadeel, Nanomed.: Nanotechnol. Biol. Med., 2014, 10, 1421.

13 R. Jin, B. Lin, D. Li and H. Ai, Curr. Opin. Pharm., 2014, 18, 18.

14 M. Su, H. Liu, L. Ge, Y. Wang, S. Ge, J. Yu and M. Yan, Electrochim. Acta, 2014, 146, 262.

15 X. Wang, X. Sun, J. Lao, H. He, T. Cheng, M. Wang, S. Wang and F. Huang, Colloids Surf., B, 2014, 122, 638.

16 X. Cao, L. Tao, S. Wen, W. Hou and X. Shi, Carbohydr. Res., 2014, 405, 70.

17 M. Adeli, S. Beyranvand and R. Kabiri, Polym. Chem., 2013, 4, 669.

18 A. Batigelli, C. Ménard-Moyou and A. Bianco, J. Mater. Chem. B, 2014, 2, 6144.

19 X. Y. Ke, V. W. L. Ng, S. J. Gao, Y. W. Tong, J. L. Hedrick and Y. Y. Yang, Biomaterials, 2014, 35, 1096.

20 A. Pourjavadi, M. Adeli and M. Yazdi, New J. Chem., 2013, 37, 295.

21 C. Y. Ang, S. Y. Tan and Y. Zhao, Org. Biomol. Chem., 2014, 12, 4776 .

22 B. K. Chegeni, A. Kakanejadifard, F. Abedi, R. Kabiri, F. Daneshnia and M. Adeli, Colloid Polym. Sci., 2014, 292, 3337.

23 S. Sunoqrot, J. Bugno, D. Lantvit, J. E. Burdette and S. Hong, J. Controlled Release, 2014, 191, 115.

24 S. J. Guillaudeu, M. E. Fox, Y. M. Haidar, E. E. Dy, F. C. Szoka and J. M. Fréchet, Bioconjugate Chem., 2008, 19, 461.

25 A. Grotzky, E. Altamura, J. Adamcik, P. Carrara, P. Stano, F. Mavelli, T. Nauser, R. Mezzenga, A. D. Schlüter and P. Walde, Langmuir, 2013, 29, 10831.

26 X. Zhang, S. Guo, R. Fan, M. Yu, F. Li, C. Zhu and Y. Gan, Biomaterials, 2012, 33, 7103.

27 H. Maeda, J. Controlled Release, 2012, 164, 138.

28 J. Fang, H. Nakamura and H. Maeda, Adv. Drug Delivery Rev., 2011, 63, 136.

29 H. S. Oberoi, F. C. Laquer, L. A. Marky, A. V. Kabanov and T. K. Bronich, J. Controlled Release, 2011, 153, 64.

30 Y. Liu, H. Miyoshi and M. Nakamura, Int. J. Cancer, 2007, $120,2527$.
31 F. Hosseini, A. Panahifar, M. Adeli, H. Amiri, A. Lascialfari, F. Orsini, M. R. Doschak and M. Mahmoudi, Colloids Surf., $B, 2013,103,652$.

32 M. Adeli, M. Ashiri, B. K. Chegeni and P. Sasanpour, J. Iran. Chem. Soc., 2013, 10, 701.

33 A. Jhavery, P. Deshpande and V. Torchilin, J. Controlled Release, 2014, 190, 352.

34 M. Ferrari, Nat. Rev. Cancer, 2005, 5, 161.

35 M. E. Davis, Z. Chen and D. M. Shin, Nat. Rev. Drug Discovery, 2008, 7, 771.

36 I. Gitsov, I. V. Berlinova and N. G. Vladimirov, J. Polym. Sci., Part A: Polym. Chem., 2015, 53, 178.

37 T. Gillich, C. Acikgöz, L. Isa, A. D. Schlüter, N. D. Spencer and M. Textor, ACS Nano, 2013, 7, 316.

38 I. Gitsov, K. L. Wooley, C. J. Hawker and J. M. Fréchet, J. Polym. Prep., 1991, 32, 631.

39 I. Gitsov, K. L. Wooley and J. M. J. Fréchet, Angew. Chem., Int. Ed. Engl., 1992, 31, 1200.

40 A. Carlmark, E. Malmström and M. Malkoch, Chem. Soc. Rev., 2013, 42, 5858.

41 I. J. Gitsov, Polym. Sci., Part A: Polym. Chem., 2008, 46, 5295.

42 K. R. Lambrych and I. Gitsov, Macromolecules, 2003, 36, 1068.

43 C. M. Dong and G. Liu, Polym. Chem., 2013, 4, 46.

44 B. K. Nanjwade, H. M. Bechra, G. K. Derkar, F. V. Manvi and V. K. Nanjwade, Eur. J. Pharm. Sci., 2009, 38, 185.

45 S. Mignani, S. El Kazzouli, M. Bousmina and J. P. Majoral, Adv. Drug Delivery Rev., 2013, 65, 1316.

46 V. Gajbhiye, V. K. Palanirajan, R. Tekade and N. K. Jain, J. Pharm. Pharmacol., 2009, 61, 989.

47 M. Adeli, A. K. Fard, F. Abedi, B. K. Chegeni and F. Bani, Nanomed.: Nanotechnol. Biol. Med., 2013, 9, 1203.

48 M. Adeli, Z. Zarnegar, A. Dadkhah, R. Hossieni, F. Salimi and A. Kanani, J. Appl. Polym. Sci., 2007, 104, 267.

49 M. W. P. L. Baars, R. Kleppinger, M. H. J. Koch and E. W. Meijer, Angew. Chem., Int. Ed., 2000, 39, 1285.

50 S. E. Stiriba, H. Kautz and H. Frey, J. Am. Chem. Soc., 2002, 124, 9698.

51 M. Krämer, J. F. Stumbé, H. Türk, S. Krause, A. Komp, L. Delineau, S. Prokhorova, H. Kautz and R. Haag, Angew. Chem., Int. Ed., 2002, 114, 4426.

52 Y. Chang, C. Park, K. T. Kim and C. Kim, Langmuir, 2005, 21, 4334 .

53 C. Zhu, C. Hard, C. Lin and I. Gitsov, J. Polym. Sci., Part A: Polym. Chem., 2005, 43, 4017.

54 I. Gitsov, in Advances in Dendritic Macromolecules, ed. G. R. Newkome, Elsevier Science, Amsterdam, 2002, vol. 5, p. 45.

55 T. M. Chapman, G. L. Hillyer, E. J. Mahan and K. A. Shaffer, J. Am. Chem. Soc., 1994, 116, 11195.

56 I. Gitsov, P. T. Ivanova and J. M. J. Fréchet, Macromol. Rapid Commun., 1994, 15, 387.

57 E. Blasco, M. Pinol and L. Oriol, Macromol. Rapid Commun., 2014, 35, 1090.

58 F. Wurm and H. Frey, Prog. Polym. Sci., 2011, 36, 1. 
59 P. M. Nguyen and P. T. Hammond, Langmuir, 2006, 22, 7825.

60 J. Zhua and X. Shi, J. Mater. Chem. B, 2013, 1, 4199.

61 S. Mignani and J. P. Majoral, New J. Chem., 2013, 37, 3337.

62 Z. Zhou, A. D'Emanuele, K. Lennon and D. Attwood, Macromolecules, 2009, 42, 7936.

63 C. Kojima, T. Suehiro, K. Watanabe, M. Ogawa, A. Fukuhara, E. Nishisaka, A. Harada, K. Kono, T. Inui and Y. Magata, Acta Biomater., 2013, 9, 5673.

64 A. R. Menjoge, M. Rangaramanujam, R. M. Kannan and D. A. Tomalia, Drug Discovery Today, 2010, 15, 171.

65 P. Singh, U. Gupta, A. Asthana and N. K. Jain, Bioconjugate Chem., 2008, 19, 2239.

66 M. H. Li, S. K. Choi, T. P. Thomas, A. Desai, K. H. Lee, A. Kotlyar, M. M. B. Holl and J. R. Baker Jr., Eur. J. Med. Chem., 2012, 47, 560.

67 Y. Chang, X. Meng, Y. Zhao, K. Li, B. Zhao, M. Zhu, Y. Li, X. Chen and J. Wang, J. Colloid Interface Sci., 2011, 363, 403.

68 W. Yang, Y. Cheng, T. Xu, X. Wang and L. P. Wen, Eur. J. Med. Chem., 2009, 44, 862.

69 A. Sousa-Herves, R. Riguera and E. Fernandez-Megia, New J. Chem., 2012, 36, 205.

70 G. Whitton and E. R. Gillies, J. Polym. Sci., Part A: Polym. Chem., 2015, 53, 148.

71 T. P. Thomas, A. K. Patri, A. Myc, M. T. Myaing, J. Y. Ye, T. B. Norris and J. R. Baker Jr., Biomacromolecules, 2004, 5, 2269.

72 M. F. Neerman, H. T. Chen, A. R. Parrish and E. E. Simanek, Mol. Pharm., 2004, 1, 390.

73 V. Torchilin, Adv. Drug Delivery Rev., 2011, 63, 131.

74 A. Buczkowski, S. Sekowski, A. Grala, D. Palecz, K. Milowska, P. Urbaniak, T. Gabryelak, H. Piekarski and B. Palecz, Int. J. Pharm., 2011, 408, 266.

75 Y. Wang, R. Guo, X. Cao, M. Shen and X. Shi, Biomaterials, 2011, 32, 3322.

76 H. Namazi and S. Jafarirad, Int. J. Pharm., 2011, 407, 167.

77 T. C. Stover, Y. S. Kim, T. L. Lowe and M. Kester, Biomaterials, 2008, 29, 359.

78 Y. Zhang, C. Xiao, M. Li, J. Ding, C. He, X. Zhuang and X. Chen, Polym. Chem., 2014, 5, 2801.

79 Z. Zhou, X. Ma, E. Jin, J. Tang, M. Sui, Y. Shen, E. A. Van Kirk, W. J. Murdoch and M. Radosz, Biomaterials, 2013, 34, 5722 .

80 Y. Li, K. Xiao, J. Luo, W. Xiao, J. S. Lee, A. M. Gonik, J. Kato, T. A. Dong and K. S. Lam, Biomaterials, 2011, 32, 6633.

81 K. Kono, C. Kojima, N. Hayashi, E. Nishisaka, K. Kiura, S. Watarai and A. Harada, Biomaterials, 2008, 29, 1664.

82 E. R. Gillies and J. M. J. Fréchet, Pure Appl. Chem., 2004, 76, 1295.

83 J. Lim and E. E. Simanek, Adv. Drug Delivery Rev., 2012, 64, 826.

84 K. Xiao, J. Luo, W. L. Fowler, Y. Li, J. S. Lee, L. Xing, R. H. Cheng, L. Wang and K. S. Lam, Biomaterials, 2009, 30, 6006 .
85 T. Negishi, F. Koizumi, H. Uchino, J. Kuroda, T. Kawaguchi, S. Naito and Y. Matsumura, Br. J. Cancer, 2006, 95, 601.

86 F. Nederberg, E. Appel, J. P. K. Tan, S. H. Kim, K. Fukushima, J. Sly, R. D. Miller, R. M. Waymouth, Y. Y. Yang and J. L. Hedrick, Biomacromolecules, 2009, 10, 1460.

87 J. Lim, S. T. Lo, S. Hill, G. M. Pavan, X. Sun and E. E. Simanek, Mol. Pharm., 2012, 9, 404.

88 G. Gaucher, R. H. Marchessault and J. C. Leroux, J. Controlled Release, 2010, 143, 2.

89 I. J. Majoros, A. Myc, T. Thomas, C. B. Mehta and J. R. Baker Jr., Biomacromolecules, 2006, 7, 572.

90 J. Luo, K. Xiao, Y. Li, J. S. Lee, L. Shi, Y. H. Tan, L. Xing, R. H. Cheng, G. Y. Liu and K. S. Lam, Bioconjugate Chem., 2010, 21, 1216.

91 Z. Poon, J. A. Lee, S. Huang, R. J. Prevost and P. T. Hammond, Nanomed.: Nanotechnol. Biol. Med., 2011, 7, 201.

92 H. Qiao, J. Li, Y. Wang, Q. Ping, G. Wang and X. Gu, Int. J. Pharm., 2013, 452, 363.

93 S. Unezaki, K. Maruyama, J. Hosoda and I. Nagai, Int. J. Pharm., 1996, 144, 11.

94 S. C. Kim, D. W. Kim, Y. H. Shim, J. S. Bang, H. S. Oh, S. Wan Kim, et al., J. Controlled Release, 2001, 72, 191.

95 P. J. Stevens, M. Sekido and R. J. A. Lee, Pharm. Res., 2004, 21, 2153.

96 H. S. Yoo and T. G. Park, J. Controlled Release, 2004, 96, 273.

97 J. F. Kukowska-Latallo, K. A. Candido, Z. Cao, S. S. Nigavekar, I. J. Majoros, T. P. Thomas, et al., Cancer Res., 2005, 65, 5317.

98 C. Chamorro, M. A. Boerman, C. J. Arnusch, E. Breukink and R. J. Pieters, Biochim. Biophys. Acta, Biomembr., 2012, 1818, 2171.

99 S. Aryal, J. J. Grailer, S. P. D. A. Steeberband and S. Gong, J. Mater. Chem., 2009, 19, 7879.

100 J. Chen, M. M. Q. Xing and W. Zhong, Polymer, 2011, 52, 933.

101 K. T. Al-Jamal, W. T. Al-Jamal, J. T. W. Wang, N. Rubio, J. Buddle, D. Gathercole, M. Zloh and K. Kostarelos, ACS Nano, 2013, 7, 1905.

102 Z. Wu, X. Zeng, Y. Zhang, N. Feliu, P. Lundberg, B. Fadeel, M. Malkoch and A. M. Nyström, J. Polym. Sci., Part A: Polym. Chem., 2012, 50, 217.

103 G. Minotti, P. Menna, E. Salvatorelli, G. Cairo and L. Gianni, Pharmacol. Rev., 2004, 56, 185.

104 Z. Deng, F. Yan, Q. Jin, F. Li, J. Wu, X. Liu and H. Zheng, J. Controlled Release, 2014, 174, 109.

105 G. Y. Li, S. Song, L. Guo and S. M. Ma, J. Polym. Sci., Part A: Polym. Chem., 2008, 46, 5028.

106 Y. Wang, X. Cao, R. Guo, M. Shen, M. Zhang, M. Zhu and X. Shi, Polym. Chem., 2011, 2, 1754.

107 E. R. Gillies and J. M. J. Fréchet, Bioconjugate Chem., 2005, 16, 361 .

108 K. Xiao, J. Luo, Y. Li, J. S. Lee, G. Fung and K. S. Lam, J. Controlled Release, 2011, 155, 272. 
109 X. Wu, X. He, L. Zhong, S. Lin, D. Wang, X. Zhu and D. Yan, J. Mater. Chem., 2011, 21, 13611.

110 X. He, X. Wu, X. Cai, S. Lin, M. Xie, X. Zhu and D. Yan, Langmuir, 2012, 28, 11929.

111 M. Adeli, N. Mirab, M. S. Alavidjeh, Z. Sobhani and F. Atyabi, Polymer, 2009, 50, 3528.

112 N. W. S. Kam, Z. Liu and H. Dai, J. Am. Chem. Soc., 2005, 127, 12492.

113 M. Maleki, M. Adeli, A. Kakanejadifard, S. Movahedi and F. Bani, Polymer, 2013, 54, 4802.

114 D. A. Yarotski, S. V. Kilina, A. A. Talin, S. Tretiak, O. V. Prezhdo, A. V. Balatsky, et al., Nano Lett., 2009, 9, 12.

115 M. Adeli, R. Soleyman, Z. Beiranvand and F. Madani, Chem. Soc. Rev., 2013, 42, 5231.

116 M. Yuksel, D. G. Colak, M. Akin, et al., Biomacromolecules, 2012, 13, 2680.

117 H. Durmaz, A. Dag, U. Tunca and G. Hizal, J. Polym. Sci., Part A: Polym. Chem., 2012, 50, 2406.

118 G. J. Bahun and A. Adronov, J. Polym. Sci., Part A: Polym. Chem., 2010, 48, 1016.

119 P. Liu, Eur. Polym. J., 2005, 41, 2693.

120 A. B. Dalton, C. Stephan, J. N. Coleman, B. McCarthy, P. M. Ajayan and S. Lefrant, J. Phys. Chem. B, 2000, 104, 10012.

121 J. Chen, H. Liu, W. A. Weimer, M. D. Halls, D. H. Waldeck and G. C. Walker, J. Am. Chem. Soc., 2002, 124, 9034.

122 M. Adeli, N. Mirab and F. Zabihi, Nanotechnology, 2009, 20, 485603.

123 C.-C. Liu, S. Sadhasivam, S. Savitha and F.-H. Lin, Talanta, 2014, 122, 195.

124 J. J. Khandare, A. Jalota-Badhwar, S. D. Satavalekar, et al., Nanoscale, 2012, 4, 837.

125 M. Adeli, S. Beyranvand and M. Hamid, J. Mater. Chem., 2012, 22, 6947.

126 A. Simonyan and I. Gitsov, Langmuir, 2008, 24, 11431.

127 I. Gitsov, K. R. Lambrych, V. A. Remnant and R. Pracitto, J. Polym. Sci., Part A: Polym. Chem., 2000, 38, 2711.

128 M. Adeli and R. Haag, J. Polym. Sci., Part A: Polym. Chem., 2006, 44, 5740.

129 Y. Hed, Y. Zhang, O. C. J. Andrén, X. Zeng, A. M. Nyström and M. Malkoch, J. Polym. Sci., Part A: Polym. Chem., 2013, 51, 3992.

130 N. Fomina, J. Sankaranarayanan and A. Almutairi, Adv. Drug Delivery Rev., 2012, 64, 1005.

131 G. Liu, W. Liu and C. M. Dong, Polym. Chem., 2013, 4, 3431.

132 L. Sun, B. Zhu, Y. Su and C. M. Dong, Polym. Chem., 2014, $5,1605$.

133 G. Liu, W. Liu and C. M. Dong, Polym. Chem., 2013, 4, 3431.

134 N. Fomina, J. Sankaranarayanan and A. Almutairi, Adv. Drug Delivery Rev., 2012, 64, 1005.

135 L. Sun, X. Ma, C. M. Dong, B. Zhu and X. Zhu, Biomacromolecules, 2012, 13, 3581.

136 O. L. P. De Jesús, H. R. Ihre, L. Gagne, J. M. J. Fréchet and F. C. Szoka Jr., Bioconjugate Chem., 2002, 13, 453.
137 E. R. Gillies and J. M. J. Fréchet, J. Am. Chem. Soc., 2002, 124, 14137.

138 E. R. Gillies, E. Dy, J. M. J. Fréchet and F. C. Szoka, Mol. Pharm., 2005, 2, 129.

139 C. Lee, E. R. Gillies, E. Dy, M. E. Fox, A. T. Cramer, J. M. J. Fréchet, E. E. Dy and F. C. Szoka, Proc. Natl. Acad. Sci. U. S. A., 2006, 45, 16649.

140 C. C. Lee, A. T. Cramer, F. C. Szoka and J. M. J. Fréchet, Bioconjugate Chem., 2006, 17, 1364.

141 J. Huang, F. Gao, X. Tang, J. Yu, D. Wang, S. Liu and Y. Li, Polym. Int., 2010, 59, 1390.

142 M. Spiess, Biochemistry, 1990, 29, 10009.

143 W. She, K. Luo, C. Zhang, G. Wang, Y. Geng, L. Li, B. He and Z. Gu, Biomaterials, 2013, 34, 1613.

144 J. del Barrio, L. Oriol, C. Sanchez, J. L. Serrano, A. Di Cicco, P. Keller, et al., J. Am. Chem. Soc., 2010, 132, 3762.

145 J. A. MacKay, M. Chen, J. R. McDaniel, W. Liu, A. J. Simnick and A. Chilkoti, Nat. Mater., 2009, 8, 993.

146 W. She, N. Li, K. Luo, C. Guo, G. Wang, Y. Geng and Z. Gu, Biomaterials, 2013, 34, 2252.

147 Y. Zhang, C. Xiao, M. Li, J. Chen, J. Ding, C. He, X. Zhuang and X. Chen, Macromol. Biosci., 2013, 13, 584.

148 Y. Bae, N. Nishiyama, S. Fukushima, H. Koyama, M. Yasuhiro and K. Kataoka, Bioconjugate Chem., 2005, 16, 122.

149 A. Shenderova, T. G. Burke and S. P. Schwendeman, Pharm. Res., 1999, 12, 241.

150 M. E. Davis, Nat. Rev. Drug Discovery, 2008, 7, 771.

151 S. Zhu, M. Hong, G. Tang, L. Qian, J. Lin, Y. Jiang and Y. Pei, Biomaterials, 2010, 31, 1360.

152 G. Mattheolabakis, E. Taoufik, S. Haralambous, M. L. Roberts and K. Avgoustakis, Eur. J. Pharm. Biopharm., 2009, 71, 190.

153 T. Boulikas and M. Vougiouka, Oncol. Rep., 2004, 11, 559.

154 S. Aryal, C. M. J. Hu and L. Zhang, ACS Nano, 2010, 4, 251.

155 D. Wang and S. J. Lippard, Nat. Rev. Drug Discovery, 2005, 4, 307.

156 M. Galanski, V. B. Arion, M. A. Jakupec and B. K. Keppler, Curr. Pharm. Des., 2003, 9, 2078.

157 K. Osada, R. J. Christie and K. Kataoka, J. R. Soc. Interface, 2009, 6, S325.

158 M. Casolaro, R. Cini, B. Del Bello, M. Ferrali and E. Maellaro, Biomacromolecules, 2009, 10, 944.

159 E. Gianasi, M. Wasil, E. G. Evagorou, A. Keddle, G. Wilson and R. Duncan, Eur. J. Cancer, 1999, 35, 994.

160 I. Haririan, M. S. Alavidjeh, M. R. Khorramizadeh, M. S. Ardestani, Z. Z. Ghane and H. Namazi, Int. J. Nanomed., 2010, 5, 63.

161 M. Adeli, F. Hakimpoor, M. Ashiri, R. Kabiri and M. Bavadi, Soft Matter, 2011, 7, 4062.

162 A. T. Naeini, M. Adeli and M. Vossoughi, Nanomed.: Nanotechnol. Biol. Med., 2010, 6, 556.

163 A. T. Naeini, M. Adeli and M. Vossoughi, Eur. Polym. J., 2010, 46, 165.

164 E. Mehdipoor, M. Adeli, M. Bavadi, P. Sasanpour and B. Rashidian, J. Mater. Chem., 2011, 21, 15456. 
165 S. Kusari, S. Zühlke and M. Spiteller, J. Nat. Prod., 2009, $72,2$.

166 M. Berrada, A. Serreqi, F. Dabbarh, A. Owusu, A. Gupta and S. Lehnert, Biomaterials, 2005, 26, 2115.

167 Y. Cheng, M. Li and T. Xu, Eur. J. Med. Chem., 2008, 43, 1791.

168 K. M. Tyner, S. R. Schiffman and E. P. Giannelis, J. Controlled Release, 2004, 95, 501.

169 L. Zhang, Y. Hu, X. Jiang, C. Yang, W. Lu and Y. H. Yang, J. Controlled Release, 2004, 96, 135.

170 K. H. Min, K. Park, Y. S. Kim, S. M. Bae, S. Lee, H. G. Jo, R. W. Park, I. S. Kim, S. Y. Jeong, K. Kim and I. C. Kwon, J. Controlled Release, 2008, 127, 208.
171 T. Schluep, J. Cheng, K. T. Khin and M. E. Davis, Cancer Chemother. Pharmacol., 2006, 57, 654.

172 S. Modi, T. X. Xiang and B. D. Anderson, J. Controlled Release, 2012, 162, 330.

173 K. Kawano, M. Watanabe, T. Yamamoto, M. Yokoyama, P. Opanasopit, T. Okano and Y. Maitani, J. Controlled Release, 2006, 112, 329.

174 S. Sadekar, G. Thiagarajan, K. Bartlett, D. Hubbard, A. Ray, L. D. McGill and H. Ghandehari, Int. J. Pharm., 2013, 456, 175.

175 S. M. Martins, B. Sarmento, C. Nunes, M. Lúcio, S. Reis and D. C. Ferreira, Eur. J. Pharm. Biopharm., 2013, 85, 488 . 